\title{
SPATIALLY NONDECAYING SOLUTIONS OF THE 2D NAVIER-STOKES EQUATION IN A STRIP
}

\author{
SERGEY ZELIK \\ Department of Mathematics, University of Surrey Guildford, GU2 7XH, United Kingdom \\ e-mail:s.zelik@surrey.ac.uk
}

(Received 23 March, 2007; accepted 24 May, 2007)

\begin{abstract}
The weighted energy theory for Navier-Stokes equations in 2D strips is developed. Based on this theory, the existence of a solution in the uniformly local phase space (without any spatial decaying assumptions), its uniqueness and the existence of a global attractor are verified. In particular, this phase space contains the 2D Poiseuille flows.
\end{abstract}

2000 Mathematics Subject Classification. 35Q30, 37L30, 76D03, 76D05

1. Introduction. It is well known that the Navier-Stokes system

$$
\left\{\begin{array}{l}
\partial_{t} u+\left(u, \nabla_{x}\right) u=v \Delta_{x} u-\nabla_{x} p+g, \\
\operatorname{div} u=0,\left.\quad u\right|_{\partial \Omega}=0,\left.\quad u\right|_{t=0}=u_{0}
\end{array}\right.
$$

in a bounded 2D domain $\Omega \subset \subset \mathbb{R}^{2}$ is well-posed and generates a dissipative semigroup $S(t)$ in the appropriate phase space (of square integrable divergent-free vector fields); see [6], [23], [24] and references therein. We also recall that these results are strongly based on the so-called energy estimate. In order to obtain this energy estimate one multiplies equation (1.1) by $u$, integrate over $\Omega$ and uses the fact that the nonlinear term disappears:

$$
\left(\left(u, \nabla_{x}\right), u\right):=\int_{x \in \Omega}\left(u(x), \nabla_{x}\right) u(x) \cdot u(x) d x \equiv 0,
$$

for every divergent-free vector field with Dirichlet boundary conditions.

In contrast to that, the situation is essentially less understood when the domain $\Omega$ is unbounded. Moreover, although there exists a highly developed theory of dissipative PDEs in unbounded domains (mainly based on the so-called weighted energy estimates, see $[7-10,18-19,27-30]$ and references therein), up to the moment, it was very difficult to extend it to the concrete Navier-Stokes problem in unbounded domains, due to several principal obstacles.

Indeed, in contrast to bounded domains, in the unbounded ones the space of square integrable (divergent-free) vector fields is not a convenient phase space, since the assumption $u \in L^{2}(\Omega)$ imposes too restrictive decay conditions on $u(x)$ as $x \rightarrow \infty$. So, under this choice of the phase space, many classical hydrodynamical

This work is partially supported by Alexander von Humboldt foundation and by the CRDF grant RUM12654-MO-05. The author is also grateful to A. Afendikov M. Efendiev and A. Mielke for stimulating discussions. 
objects, like Poiseuille flows, Couette-Taylor flows, Kolmogorov flows etc. are automatically out of consideration. Thus, following the general theory mentioned above, it is reasonable to replace the assumption $u \in L^{2}(\Omega)$ by more relevant one: $u \in L_{b}^{2}(\Omega)$ where the uniformly local Sobolev spaces $W_{b}^{l, p}(\Omega)$ are defined via the following standard expression:

$$
W_{b}^{l, p}(\Omega):=\left\{u \in D^{\prime}(\Omega), \quad\|u\|_{W_{b}^{l, p}(\Omega)}:=\sup _{x_{0} \in \Omega}\|u\|_{W^{l, p}\left(\Omega \cap B_{x_{0}}^{1}\right)}<\infty\right\} .
$$

Here $B_{x_{0}}^{1}$ denotes the ball of radius one of $\mathbb{R}^{2}$ centred at $x_{0} \in \mathbb{R}^{2}$ and $W^{l, p}$ means the classical Sobolev space (see Section 1 for details). But here arises the main difficulty: how to obtain a priori estimates for the solution $u(t)$ in the uniformly local spaces?

Indeed, since $u(t)$ is not square integrable any more, we cannot multiply (1.1) simply by $u$ and use identity (1.2) (the integrals do not have sense). So, following the general strategy, we need to multiply it by $\phi u$ where $\phi=\phi(x)$ is an appropriate weight function. But in that case the nonlinear term does not vanish and produces the additional cubic term like $\phi^{\prime} u^{3}$. We note that this cubic term is not sign-defined and the remaining terms in the energy equality are at most quadratic with respect to $u$, so it was not clear how to control this cubic term in order to produce reasonable a priori estimates.

Another obstacle is related with the fact that $\phi u$ is not divergent free, so the pressure $p$ does not disappear in the weighted energy equality and one should be also able to control the term $\left(\phi^{\prime} p, u\right)$. Of course, this problem is closely related to finding the reasonable extension of the Helmholtz projector (to divergent free vector fields) to uniformly local spaces.

The above mentioned difficulties stimulated the developing of the alternative methods to handle the Navier-Stokes equations in unbounded domains. In particular, rather helpful is the so-called vorticity equation

$$
\partial_{t} \omega-\Delta_{x} \omega+\left(u, \nabla_{x}\right) \omega=\partial_{x_{2}} g_{1}-\partial_{x_{1}} g_{2}
$$

where $\omega:=\partial_{x_{2}} u_{1}-\partial_{x_{1}} u_{2}$. Indeed, if $\Omega$ does not contain boundary, e.g. $\Omega=\mathbb{R}^{2}$ or $\Omega=\mathbb{S}^{1} \times \mathbb{R}$ where $\mathbb{S}^{1}$ is a circle (like in the Kolmogorov problem), the maximum principle applied to (1.3) allows us to obtain global a priori estimates for the vorticity $\omega$ which, together with the accurate analysis of the explicit formulae for the Helmholtz projectors, allow us to obtain the global in time a priori estimates for the solution $u(t)$ and, thus, to prove the global solvability of the Navier-Stokes equation in the uniformly local phase spaces (see [2] and [12]). Unfortunately, the a priori estimate for vorticity obtained from the maximum principle grows linearly in time, so all of the further estimates will also grow in time (to the best of our knowledge, for the case $\Omega=\mathbb{R}^{2}$, it gives double exponential $\left(\sim e^{C e^{C t}}\right)$ growth rate and polynomial $\left(\sim t^{3}\right)$ growth rate for $\left.\Omega=\mathbb{S}^{1} \times \mathbb{R}\right)$. The other essential drawback is that this method seems to be non-applicable to the problems with boundary, e.g. in the case where $\Omega$ is a cylindrical domain.

Another attractive possibility to avoid direct weighted energy estimates is to use the bifurcation analysis. Indeed, in the situation where the basic steady state of the NavierStokes problem is slightly above the instability threshold, the solutions remaining close to that steady state can be described in terms of the so-called modulation equations which are essentially simpler than the initial Navier-Stokes problem (usually it is Ginzburg-Landau or Swift-Hohenberg equations); see [1, 13-15, 17] and references 
therein. Since the well-posedness and dissipativity of these modulation equations is well-understood, the standard perturbation methods sometimes allows us to obtain global in time estimates for solutions of the initial Navier-Stokes problem starting from the small neighborhood of the basic steady state. In particular, the global existence and dissipativity of such solutions for the 3D Couette-Taylor flow is obtained in [21] and "almost global solvability" (on the exponentially long with respect to perturbation parameter time interval) for the case of Poiseuille flow can be found in [22].

It is worth emphasizing that, in the case where the domain $\Omega \subset \mathbb{R}^{2}$ possesses the Friedrich's inequality

$$
\|u\|_{L^{2}(\Omega)}^{2} \leq \lambda_{1}\left\|\nabla_{x} u\right\|_{L^{2}(\Omega)}^{2}, \quad u \in W_{0}^{1,2}(\Omega)
$$

with positive $\lambda_{1}$ and under the restrictive assumption that $u$ is square integrable, all of the above mentioned obstacles disappear and Navier-Stokes problem (1.1) possesses a standard (unweighted) energy theory similar to the case of bounded domains, see [5], [24]. We also mention the survey paper [3] on existence of spatially decaying solutions of the Navier-Stokes problem in various domains (not necessarily satisfying (0.4)); see also [11] and [26].

The main aim of the present paper is to develop weighted energy theory for the 2D Navier-Stokes problems in a strip $\Omega:=\mathbb{R} \times(-1,1),\left(x_{1}, x_{2}\right) \in \Omega$ overcoming the obstacles mentioned above. For simplicity, we will mainly consider the model NavierStokes problem

$$
\left\{\begin{array}{l}
\partial_{t} u+\left(u, \nabla_{x}\right) u=\Delta_{x} u-\nabla_{x} p+g \\
\operatorname{div} u=0,\left.u\right|_{\partial \Omega}=0,\left.\quad u\right|_{t=0}=u_{0}
\end{array}\right.
$$

with $v=1$ (the case of arbitrary $v$ can be reduced to $v=1$ by the appropriate scaling, see the end of Section 8). Moreover, in order to make problem (1.5) well posed, we need to add the average flux condition:

$$
\left(S u_{1}\right)\left(t, x_{1}\right):=1 / 2 \int_{-1}^{1} u_{1}\left(t, x_{1}, x_{2}\right) d x_{2} \equiv c
$$

where $c \in \mathbb{R}$ is a given constant (assumption (1.3) can be considered as a kind of "boundary conditions" at $\left.x_{1}= \pm \infty\right)$.

The main result of the paper is a comprehensive study of the Navier-Stokes problem (1.5), (1.6) in the uniformly local spaces (i.e. requiring the solution $u(t)$ be only bounded as $x_{1} \rightarrow \pm \infty$, no decaying conditions are imposed). In particular, we prove the existence of a solution, its uniqueness and regularity, dissipativity and existence of a locally compact global attractors for the Navier-Stokes problem (1.5), (1.6). We emphasize that, in contrast to the previous results on this topic, our phase space contains all of the Poiseuille flows and all known structures bifurcating from them. Moreover, our result allows us to embed the 2D Navier-Stokes problem in a strip into a general scheme of investigating dissipative PDEs in unbounded domains mentioned above, including the study of the dimension and Kolmogorov's entropy of attractors, topological entropies, spatial and temporal chaos, etc. We return to these questions in the forthcoming paper [31].

The paper is organized as follows. We recall in Sections 2 and 3 some basic facts on the theory of weighted spaces and the regularity of elliptic boundary value problems in these spaces which will be systematically used throughout the paper. 
Section 4 is devoted to study the Helmholtz projector $\Pi$ and the Stokes operator $A:=\Pi \Delta_{x}$ in weighted and uniformly local Sobolev spaces. The results of this section are similar to [4] and [5] (and are, actually, inspired by these papers).

In Section 5, we study the auxiliary linear non-divergent free problem

$$
-\partial_{t} v=\Delta_{x} v+\nabla_{x} q,\left.\quad \Pi v\right|_{t=T}=0, \quad \operatorname{div} u=\phi^{\prime} u,\left.\quad v\right|_{\partial \Omega}=0
$$

where $\phi(x)$ is the appropriate weight function and $u(t)$ is a solution of the Navier-Stokes problem. This auxiliary problem is necessary in order to overcome the obstacle related with the appearance of the term containing pressure in the weighted energy equality. Roughly speaking, we will multiply equation (1.5) by the function $\phi u(t)-v(t)$ where $v$ solves (1.7). Then, since $\operatorname{div} u(\phi u-v)=0$ the pressure term disappears (and the derivative of our weights will be small, so the corrector $v$ will be also small and will not produce any essential difficulties in its estimating, see Sections 5 and 6 for the details).

We note that it is not clear how to overcome this obstacle in more simple way. Indeed, the "most natural" multiplication by $\Pi(\phi u)$ does not work since $\Pi(\phi u)$ has nonzero trace at the boundary which leads to additional uncontrollable boundary terms under the integration by parts in $\left(\Delta_{x} u, \Pi(\phi u)\right)$. Another possibility is to construct a new "projector" $Q$ to divergent free vector fields which preserves the boundary conditions, and multiply the equation by $Q(\phi u)$. This, however, leads to essential difficulties with the term $\left(\partial_{t} u, Q(\phi u)\right)$ which should be a complete time derivative from something. We also note that the multiplication of the equation by the combination of $\phi \partial_{t} u$ and $\phi \Pi \Delta_{x} u$ (as in [4] and [5]) is useless for us, since it works only if the unweighted $L^{2}$-norm of $\Delta_{x} u$ is a priori known.

In Section 6 we overcome the main obstacle to the weighted energy theory for Navier-Stokes equations - the cubic term $\phi^{\prime} u^{3}$ mentioned above. In order to do so, we use the special weights

$$
\theta_{\varepsilon, x_{0}}(x):=\left(1+\varepsilon^{2}\left|x-x_{0}\right|^{2}\right)^{1 / 2}
$$

with very small $\varepsilon$ which actually depends on the solution $u$ considered. Then, the careful analysis of the obtained weighted energy inequality allows us to obtain the globally in time bounded a priori estimate of the $L_{b}^{2}$-norm of $u(t)$. Based on this a priori estimate, we then establish the existence of such solution. In fact, we first consider the case of zero flux $c=0$ (see Theorem 6.5) and, after that, reduce the general case to that particular one using the trick with the auxiliary "energy stable" equilibrium (see Theorem 6.6).

The uniqueness of such a solution is verified in Section 7 (see Theorem 7.1). Moreover, we also verify here the $L_{b}^{2}-W_{b}^{1,2}$ smoothing property for that solution which is necessary for global attractors (see Theorem 7.4).

Finally, in Section 8, we prove the dissipative estimate (=existence of an absorbing ball) for solutions of Navier-Stokes problem in the uniformly local phase space (Theorem 8.1) and establish the existence of a global attractor $\mathcal{A}$. Moreover, using the scaling arguments, we obtain the following estimate for the size of attractor in $L_{b}^{2}$-norm in terms of the kinematic viscosity $v$ :

$$
\|\mathcal{A}\|_{L_{b}^{2}(\Omega)} \leq C v^{-3}\left(c^{3} v+\|g\|_{L_{b}^{2}(\Omega)}^{2}+v^{4}\right)
$$

where the constant $C$ is independent of $v, c$ and $g$. We recall that in bounded domains (in square integrable case), the best known estimate is the following one:

$$
\|\mathcal{A}\|_{L^{2}(\Omega)} \leq C v^{-1}\|g\|_{L^{2}(\Omega)} .
$$


We see that, although estimate (1.9) is "worse" than (1.10), it remains polynomial as $v \rightarrow 0$ (with a reasonable degree 3 ). Thus, our method is not "extremely rough" and can be used in order to obtain reasonable quantitative bounds for the solutions.

To conclude, we mention that our method seems to be applicable to more general 2D domains satisfying (1.4) and even to 3D cylindrical domains (of course, up to the uniqueness problem). We return to these topics somewhere else.

2. Functional spaces. In this section, we briefly recall the definitions and basic properties of weight functions and weighted functional spaces which will be systematically used throughout the paper (see also [9], [28] for more details). We start with the class of admissible weight functions.

DEFINITION 2.1. A function $\phi \in C_{l o c}\left(\mathbb{R}^{n}\right)$ is a weight function of exponential growth rate $\mu>0$ if the following inequalities hold:

$$
\phi(x+y) \leq C_{\phi} \phi(x) e^{\mu|y|}, \phi(x)>0,
$$

for all $x, y \in \mathbb{R}^{n}$.

The following proposition collects the evident properties of these weights.

Proposition 2.2. (1). Let $\phi$ be a weight function with exponential growth rate $\mu$. Then, for every $\varepsilon>\mu, \phi$ is a weight function of exponential growth rate $\varepsilon$ (with the same constant $C_{\phi}$ ).

(2). Let $\phi$ and $\psi$ be weight functions of exponential growth rate $\mu$. Then the functions $\Psi_{1}=\phi(x) \psi(x)$ and $\Psi_{2}=\phi(x) / \psi(x)$ are weight functions of exponential growth rate $2 \mu$ with the constant $C_{\Psi_{i}} \leq C_{\phi} C_{\psi}$.

(3). Let $\phi$ be a weight function of exponential growth rate $\mu$ and let $\psi \in C_{\text {loc }}\left(\mathbb{R}^{n}\right)$ satisfy

$$
C_{1} \phi(x) \leq \psi(x) \leq C_{2} \phi(x), \quad x \in \mathbb{R}^{n}
$$

Then $\psi$ is also a weight function of exponential growth rate $\mu$ and $C_{\psi} \leq C_{1}^{-1} C_{2} C_{\phi}$.

(4). Let $\varepsilon>0$ and $\phi(x)$ be a weight function of exponential growth rate $\mu$. Then the function $\phi_{\varepsilon}(x):=\phi(\varepsilon x)$ is of exponential growth rate $\varepsilon \mu$ and with $C_{\phi_{\varepsilon}}=C_{\phi}$.

All of the assertions of the proposition are simple corollaries of estimate (2.1).

The natural example of such weights is the following one:

$$
\phi_{\mu, x_{0}}(x):=e^{-\mu\left|x-x_{0}\right|}, \quad x_{0} \in \mathbb{R}^{n}, \quad \mu \in \mathbb{R} .
$$

Obviously, they are of exponential growth rate $|\mu|$ and the constant $C_{\phi_{\mu, x_{0}}}=1$ (independent of $x_{0} \in \mathbb{R}^{n}$ ). However, these weights are nonsmooth at $x=x_{0}$. In order to overcome this drawback, it is natural to use the following equivalent weights:

$$
\varphi_{\mu, x_{0}}(x):=e^{-\mu \sqrt{1+\left|x-x_{0}\right|^{2}}}, \quad x_{0} \in \mathbb{R}^{n} .
$$

Indeed, since $|x| \leq \sqrt{x^{2}+1} \leq|x|+1$, then these weights satisfy

$$
e^{-|\mu|} \phi_{\mu, x_{0}}(x) \leq \varphi_{\mu, x_{0}}(x) \leq e^{|\mu|} \phi_{\mu, x_{0}}(x), \quad x \in \mathbb{R}^{n}
$$


and, consequently, $\varphi_{\mu, x_{0}}$ are also weight functions of exponential growth rate $\mu$ (with $C_{\varphi_{\mu, x_{0}}}=e^{2|\mu|}$ ). Moreover, in contrast to (2.3) these weights are smooth and satisfy, for $\mu \leq 1$, the additional obvious inequality

$$
\left|D_{x}^{k} \varphi_{\mu, x_{0}}(x)\right| \leq C_{k}|\mu| \varphi_{\mu, x_{0}}(x), \quad x \in \mathbb{R}^{n}
$$

where $k \in \mathbb{N}, D_{x}^{k}$ denotes a collection of all $x$-derivatives of order $k$ and the constant $C_{k}$ is independent of $x$ and $\mu$. This inequality is crucial for obtaining the regularity estimates in weighted spaces (see [9-10, 27-30] and Section 3 below).

Another important class of weight functions is the so-called polynomial class:

$$
\theta_{x_{0}}^{m}(x):=\left(1+\left|x-x_{0}\right|^{2}\right)^{-m / 2}, \quad m \in \mathbb{R} .
$$

It is not difficult to verify that these weights are of exponential growth rate $\mu$ for every $\mu>0$ with the constant $C_{\theta_{m, x_{0}}}$ depending on $\mu$ and $m$, but independent of $x_{0} \in \Omega$.

We now introduce a class of weighted Sobolev spaces in a regular unbounded domain $\Omega$ associated with weights introduced above. Since we actually need below only the case where $\Omega:=\mathbb{R} \times(-1,1)$ is a strip which obviously has regular boundary, in order to avoid the technicalities we do not formulate precise assumptions on the boundary $\partial \Omega$ (which can be found e.g. in [9] or [10]).

DEFINITION 2.3. Let $\Omega$ be a regular domain and let $\phi$ be a weight function of exponential growth rate. Then, for every $1 \leq p \leq \infty$, we set

$$
L_{\phi}^{p}(\Omega):=\left\{u \in L_{l o c}^{p}(\Omega), \quad\|u\|_{L_{\phi}^{p}}^{p}:=\int_{\Omega} \phi(x)^{p}|u(x)|^{p} d x<\infty\right\}
$$

and

$$
L_{b, \phi}^{p}(\Omega):=\left\{u \in L_{l o c}^{p}(\Omega), \quad\|u\|_{L_{b, \phi}^{p}}:=\sup _{x_{0} \in \Omega}\left(\phi\left(x_{0}\right)\|u\|_{L^{p}\left(\Omega \cap B_{x_{0}}^{1}\right)}\right)<\infty\right\} .
$$

Here and below $B_{x_{0}}^{r}$ denotes an $r$-ball of $\mathbb{R}^{n}$ centred at $x_{0}$ and we write $L_{b}^{p}$ instead of $L_{b, 1}^{p}$.

Moreover, for every $l \in \mathbb{N}$, we define the weighted Sobolev spaces $W_{\phi}^{l, p}(\Omega)$ and $W_{b, \phi}^{l, p}(\Omega)$ as spaces of distributions whose derivatives up to order $l$ belong to $L_{\phi}^{p}(\Omega)$ and $L_{b, \phi}^{p}(\Omega)$ respectively.

Furthermore, the weighted Sobolev spaces $W_{\phi}^{l, p}(\partial \Omega)$ and $W_{b, \phi}^{l, p}(\partial \Omega)$ on the boundary $\partial \Omega$ can be defined analogously, only the integral over $\Omega$ (resp. supremum in (2.9)) in (2.8) should be naturally replaced by the integral (resp. supremum) over the boundary $\partial \Omega$, see [9], [10].

REMARK 2.4. In the sequel, we will also use the functions $u(t)$ with values in the weighted Sobolev spaces defined above. In slight abuse of the notation, we denote by $L_{b}^{p}\left(\mathbb{R}, W_{b}^{l, p}\right)$ the space generated by the following norm:

$$
\|u\|_{L_{b}^{p}\left(\mathbb{R}, W_{b}^{l, p}\right)}:=\sup _{x_{0} \in \Omega} \sup _{T \in \mathbb{R}}\|u\|_{L^{p}\left([T, T+1], W^{l, p}\left(\Omega \cap B_{x_{0}}^{1}\right)\right)} .
$$

The following proposition collects some useful facts on the spaces introduced above. 
PROPOSITION 2.5. Let $\Omega$ be a regular domain and $\phi$ be a weight of exponential growth rate $\mu$.

(1) For every $r>0$ and every $u \in L_{\phi}^{p}(\Omega), 1 \leq p<\infty$,

$$
C_{r}^{-1}\|u\|_{L_{\phi}^{p}(\Omega)} \leq\left(\int_{x_{0} \in \Omega} \phi^{p}\left(x_{0}\right)\|u\|_{L^{p}\left(\Omega \cap B_{x_{0}}^{r}\right)}^{p} d x_{0}\right)^{1 / p} \leq C_{r}\|u\|_{L_{\phi}^{p}(\Omega)}
$$

where the constant $C_{r}$ depends on $r, \mu$ and on the constant $C_{\phi}$ from eq (2.1), but is independent of $u$ and of the concrete choice of the weight $\phi$.

(2) For every $\alpha>\mu$, every $q \in[1, \infty]$ and every $u \in L_{\phi}^{1}(\Omega)$, we have

$$
\left(\int_{x_{0} \in \Omega} \phi\left(x_{0}\right)^{q}\left(\int_{x \in \Omega} e^{-\alpha\left|x-x_{0}\right|}|u(x)| d x\right)^{q} d x_{0}\right)^{1 / q} \leq C_{\alpha}\|u\|_{L_{\phi}^{1}(\Omega)}
$$

where the constant $C_{\alpha}$ depends on $\alpha, \mu$ and on the constant $C_{\phi}$, but is independent of $u$ and of the concrete choice of $\phi$ and $q$.

(3) For every $\alpha>\mu$ and every $u \in L_{b, \phi}^{p}(\Omega)$, we have

$$
C_{\alpha}^{-1}\|u\|_{L_{b, \phi}^{p}(\Omega)}^{p} \leq \sup _{x_{0} \in \Omega}\left\{\phi\left(x_{0}\right)^{p} \int_{x \in \Omega} e^{-\alpha p\left|x-x_{0}\right|}|u(x)|^{p} d x\right\} \leq C_{\alpha}\|u\|_{L_{b, \phi}^{p}(\Omega)}^{p}
$$

where the constant $C_{\alpha}$ depends on $\alpha, \mu$ and on the constant $C_{\phi}$, but is independent of $u$ and of the concrete choice of $\phi$.

The proof of these estimates is given in [9] (see also [10], [26]).

REMARK 2.6. As we will see below, estimate (2.11) allows us to reduce the proofs of embedding and interpolation theorems for weighted Sobolev spaces to the classical unweighted case in a bounded domain. Estimates (2.12) and (2.13) allow, in turn, to obtain the elliptic regularity in weighted spaces with arbitrary weights of exponential growth rate if the analogous result for the special weights $e^{-\alpha\left|x-x_{0}\right|}$ (or which is the same, for the equivalent smooth weights (2.4)) is known; see Section 3. Moreover, these estimates allow us to control the dependence of the constants in embedding, interpolation and regularity theorems on the concrete choice of the weights which is crucial for our study of the nondecaying solutions of NS equations.

We need now to introduce also the weighted Sobolev spaces with fractional derivatives. To this end, we first recall that in the unweighted case the space $W^{l+s, p}(\Omega)$ for $s \in(0,1)$ and $l \in \mathbb{Z}_{+}$is usually defined via

$$
\|u\|_{W^{l+s, p}(\Omega)}^{p}:=\|u\|_{W^{l, p}(\Omega)}^{p}+\int_{x \in \Omega} \int_{y \in \Omega} \frac{\left|D_{x}^{l} u(x)-D_{x}^{l} u(y)\right|^{p}}{|x-y|^{n+s p}} d x d y
$$

and, for negative $l$, the space $W^{l, p}(\Omega)$ is defined as a conjugate space to $W_{0}^{-l, q}(\Omega)$ where $1 / p+1 / q=1$, see [16], [25]. Then, estimate (2.11) justifies the following definition.

DEFINITION 2.7. Let $\Omega$ be a regular domain and $\phi$ be a weight function of exponential growth rate. For every $1<p \leq \infty$ and every $l \in \mathbb{R}$, we define the space $W_{\phi}^{l, p}(\Omega)$ as a subspace of distributions for which the following norm is finite:

$$
\|u\|_{W_{\phi}^{l, p}(\Omega)}^{p}:=\int_{x_{0} \in \Omega} \phi\left(x_{0}\right)^{p}\|u\|_{W^{l, p}\left(\Omega \cap B_{x_{0}}^{r}\right)}^{p} d x_{0}
$$


where $r$ is some positive number (it is not difficult to verify that this space is independent of $r$ ). Analogously the norm in $W_{b, \phi}^{l, p}$ is defined via

$$
\|u\|_{W_{b, \phi}^{l, p}(\Omega)}^{p}:=\sup _{x_{0} \in \Omega}\left\{\phi\left(x_{0}\right)^{p}\|u\|_{W^{l, p}\left(\Omega \cap B_{x_{0}}^{r}\right)}^{p}\right\} .
$$

For simplicity, we fix $r=1$ in definitions (2.15) and (2.16) of the weighted norms.

Indeed, according to (2.11), we see that, for $l \in \mathbb{Z}_{+}$the spaces thus defined coincide with the spaces from Definition 2.1. Moreover, it is not difficult to verify, using the explicit formula (2.14), that in the unweighted case $\phi=1$, the norm (2.15) is equivalent to $(2.14)$.

The following proposition describes the weighted negative Sobolev spaces in terms of conjugate spaces.

Proposition 2.8. Let $\Omega$ be a regular domain and let $\phi$ be a weight function of exponential growth rate $\mu$. Then, for every $l>0$, and every $1<p, q<\infty$ with $1 / p+$ $1 / q=1$,

$$
W_{\phi}^{-l, p}(\Omega)=\left[W_{0, \phi^{-1}}^{l, q}(\Omega)\right]^{*}
$$

where $W_{0, \phi}^{l, q}(\Omega)$ denotes the closure of $C_{0}^{\infty}(\Omega)$ in the $W_{\phi}^{l, q}$-norm and $*$ means the conjugate space (with respect to the standard inner product in $L^{2}(\Omega)$ ). Moreover,

$$
C_{1}\|u\|_{W_{\phi}^{-l, p}(\Omega)} \leq\|u\|_{\left[W_{0, \phi^{-1}}^{l, q}(\Omega)\right]^{*}} \leq C_{2}\|u\|_{W_{\phi}^{-l, p}(\Omega)}
$$

where the constants $C_{1}$ and $C_{2}$ depend on $\mu, l, p$ and $C_{\phi}$, but are independent of the concrete choice of $u$ and $\phi$.

Proof. In order to avoid the technicalities, we give below the proof of (2.18) only for the case of a cylindrical domain $\Omega:=\mathbb{R} \times \omega$ where $\omega$ is a smooth bounded domain of $\mathbb{R}^{n-1}$ (only that case will be used in the sequel) although the slightly modified proof works for a general regular domain. In that particular case, we can restrict ourselves to consider only one dimensional weights $\phi \in C_{\text {loc }}(\mathbb{R})$. Indeed, since $\omega$ is bounded, (2.1) implies that

$$
C_{1} \phi\left(s, \xi_{0}\right) \leq \phi(s, \xi) \leq C_{2} \phi\left(s, \xi_{0}\right), \quad s \in \mathbb{R}, \quad \xi \in \omega
$$

where $\xi_{0} \in \omega$ is some fixed point and, consequently, the weight $\phi(s, \xi)$ is equivalent to $\phi_{\xi_{0}}(s):=\phi\left(s, \xi_{0}\right)$. Moreover, it is more convenient to use, instead of balls $B_{x_{0}}^{r}$, the finite cylinders $\Omega_{s}:=(s, s+1) \times \omega$, i.e. to define the norm in $W_{\phi}^{l, p}(\Omega)$ via

$$
\|u\|_{W_{\phi}^{l, p}(\Omega)}^{p}=\int_{s \in \mathbb{R}} \phi(s)^{p}\|u\|_{W^{l, p}\left(\Omega_{s}\right)}^{p} d s
$$

(since the norms (2.15) are equivalent for different $r$ and $\omega$ is bounded then (2.15) and (2.20) are also equivalent). 
We first verify the right inequality of (2.18). To this end, we introduce a partition of unity $\left\{\psi_{y}\right\}_{y \in \mathbb{R}} \in C_{0}^{\infty}(\mathbb{R})$ such that

$$
\begin{aligned}
& \operatorname{supp} \psi_{y} \subset(y, y+1), \\
& \int_{y \in \mathbb{R}} \psi_{y}(s) d y \equiv 1, \\
& \left|D_{s}^{k} \psi_{y}(s)\right| \leq C_{k},
\end{aligned}
$$

where the constant $C_{k}$ is independent of $s \in \mathbb{R}$ (obviously such a partition of unity exists and can be chosen in a smooth way with respect to $y \in \mathbb{R})$.

Now let $u \in\left[W_{0, \phi^{-1}}^{l, q}(\Omega)\right]^{*}$ be a functional over $W_{0, \phi^{-1}}^{l, q}(\Omega)$ and let $v$ be an arbitrary test function from that space. Then, using (2.21) and Hölder inequality, we have

$$
\begin{aligned}
|\langle u, v\rangle| & \leq \int_{y \in \mathbb{R}}\left|\left\langle u, \psi_{y} v\right\rangle\right| d y \leq \int_{y \in \mathbb{R}}\|u\|_{W^{-l, p}\left(\Omega_{y}\right)}\left\|\psi_{y} v\right\|_{W^{l, q}\left(\Omega_{y}\right)} d y \\
& \leq C \int_{y \in \mathbb{R}} \phi(y)\|u\|_{W^{-l, p}\left(\Omega_{y}\right)} \cdot \phi(y)^{-1}\|v\|_{W^{l, q}\left(\Omega_{y}\right)} d y \leq C\|u\|_{W_{\phi}^{-l, p}(\Omega)}\|v\|_{W_{\phi^{-1}}^{l, q}(\Omega)}
\end{aligned}
$$

which, together with the definition of the norm in a conjugate space gives the right-hand side of inequality (2.18).

Let us now verify the left-hand side of that inequality. Indeed, let $u \in W_{\phi}^{-l, p}(\Omega)$. We fix a family of functions $v_{y} \in W_{0}^{l, q}\left(\Omega_{y}\right)$, such that

$$
\left\langle u, v_{y}\right\rangle=\|u\|_{W^{-l, p}\left(\Omega_{y}\right)}\left\|v_{y}\right\|_{W^{l, q}\left(\Omega_{y}\right)}
$$

and normalize these functions as follows:

$$
\left\|v_{y}\right\|_{W^{l, q}\left(\Omega_{y}\right)}=\phi(y)^{p}\|u\|_{W^{-l, p}\left(\Omega_{y}\right)}^{p-1} .
$$

Since the spaces $W^{l, q}\left(\Omega_{y}\right)$ are uniformly convex, these family are uniquely defined and, moreover, continuous with respect to $y \in \mathbb{R}$.

Let us also define the function $v(x)$ as follows

$$
v(x):=\int_{y \in \mathbb{R}} v_{y}(x) d y .
$$

We claim that $v \in W_{0, \phi^{-1}}^{l, q}(\Omega)$. Indeed, since $v_{y} \in W_{0}^{l, q}\left(\Omega_{y}\right)$, it can be naturally continued by zero to the function $v_{y} \in W_{0}^{l, q}(\Omega)$ with supp $v_{y} \subset \Omega_{y}$. Thus, the integral (2.25) is well posed and defines a function $v \in W_{l o c}^{l, q}(\bar{\Omega})$ vanishing at the boundary $\partial \Omega$. So, we only need to estimate the $W_{\phi^{-1}}^{l, q}(\Omega)$-norm of it.

Using now the fact that $\left\|v_{y}\right\|_{W^{l, q}\left(\Omega_{s}\right)}=0$ if $|s-y| \geq 1$, we have

$$
\begin{aligned}
\|v\|_{W^{l, q}\left(\Omega_{s}\right)} & \leq \int_{|s-y| \leq 1}\left\|v_{y}\right\|_{W^{l, q}\left(\Omega_{y}\right)} d y=\int_{|s-y| \leq 1} \phi(y)^{p}\|u\|_{W^{-l, p}\left(\Omega_{y}\right)}^{p-1} d y \\
& \leq C \phi(s)^{p} \int_{|s-y| \leq 1}\|u\|_{W^{-l, p}\left(\Omega_{y}\right)}^{p-1} d y \leq C_{1} \phi(s)^{p} \int_{y \in \mathbb{R}} e^{-\alpha|s-y|}\|u\|_{W^{-l, p}\left(\Omega_{y}\right)}^{p-1} d y
\end{aligned}
$$


where the constant $\alpha>2 p \mu / q$ can be arbitrary (here we have implicitly used (2.1) in order to estimate $\phi(y)$ via $\phi(s))$. Taking the $q$-th power of both sides of that relation, applying the Hölder inequality and using that $q(p-1)=p$, we arrive at

$$
\phi(s)^{-q}\|v\|_{W^{l, q}\left(\Omega_{s}\right)}^{q} \leq C \phi(s)^{p} \int_{y \in \mathbb{R}} e^{\alpha q|s-y| / 2}\|u\|_{W^{-l, p}\left(\Omega_{y}\right)}^{p} d y .
$$

Integrating this relation over $s \in \mathbb{R}$ and using (2.12), we finally obtain

$$
\|v\|_{W_{\phi^{-1}}^{l, q}(\Omega)}^{q} \leq C_{2}\|u\|_{W_{\phi}^{-l, p}(\Omega)}^{p} .
$$

We are now ready to finish the proof of the proposition. Indeed, due to (2.23)-(2.25), we have

$$
\langle u, v\rangle=\int_{y \in \Omega}\|u\|_{W^{-l, p}\left(\Omega_{y}\right)}\left\|v_{y}\right\|_{W^{l, q}\left(\Omega_{y}\right)} d y=\|u\|_{W_{\phi}^{-l, p}(\Omega)}^{p}
$$

and, consequently, due to (2.27),

$$
\|u\|_{\left[W_{0, \phi^{-1}}^{l, q}(\Omega)\right]^{*}} \geq \frac{\langle u, v\rangle}{\|v\|_{W_{\phi^{-1}}^{l, q}(\Omega)}} \geq C\|u\|_{W_{\phi}^{-l, p}(\Omega)}^{p(1-1 / q)} .
$$

Since $p(1-1 / q)=1,(2.28)$ implies the left-hand side of inequality (2.18). Proposition 2.8 is proved.

Remark 2.9. Proposition 2.8 shows, in particular, that in the case $\phi=1$, the spaces $W^{l, p}(\Omega)$ introduced in Definition 2.7 coincide with the standard Sobolev spaces for any $l \in \mathbb{R}$. Moreover, arguing analogously to the proof of Proposition 2.8, one can verify the interpolation representation of the weighted spaces $W_{\phi}^{l+\alpha, p}(\Omega)$ with fractional derivatives $(l \in \mathbb{Z}, \alpha \in(0,1))$

$$
W_{\phi}^{l+\alpha, p}(\Omega)=\left(W_{\phi}^{l, p}(\Omega), W_{\phi}^{l+1, p}(\Omega)\right)_{\alpha, p}
$$

in a complete analogy with the unweighted case, see e.g. [25].

We now recall also the embedding and trace theorems for the weighted functional spaces.

Proposition 2.10. Let $\Omega$ be a regular domain and $\phi$ be a weight function of exponential growth rate $\mu$. Then

(1) For every $1<p_{1} \leq p_{2}<\infty$ and every $0 \leq l_{2} \leq l_{1}$ satisfying

$$
\frac{1}{p_{2}}-\frac{l_{2}}{n} \geq \frac{1}{p_{1}}-\frac{l_{1}}{n}
$$

there is a continuous embedding $W_{\phi}^{l_{1}, p_{1}}(\Omega) \subset W^{l_{2}, p_{2}}(\Omega)$ and the norm of the embedding operator depends on $l_{i}, p_{i}, \mu$ and $C_{\phi}$, but is independent of the concrete form of the weight function $\phi$. If the inequality (2.30) is strict, then we can take also $p_{2}=\infty$.

(2) For every $m \in \mathbb{Z}_{+}, 1<p<\infty$ and $l>m+1 / p$ the trace operator $\Pi_{\partial \Omega}^{m}$

$$
\Pi_{\Omega}^{m} u:=\left(\left.u\right|_{\partial \Omega},\left.\partial_{n} u\right|_{\partial \Omega}, \cdots,\left.\partial_{n}^{m} u\right|_{\partial \Omega}\right)
$$

(where $\partial_{n} u$ denotes the normal derivative of the function $u$ at the boundary $\partial \Omega$ ) maps $W_{\phi}^{l, p}(\Omega)$ to $\otimes_{k=0}^{m} W_{\phi}^{l-k-1 / p, p}(\partial \Omega)$ and there exists the associated extension operator 
$\left[\Pi_{\partial \Omega}^{m}\right]^{-1}$ (right inverse to $\Pi_{\partial \Omega}^{m}$ ) and the norms of that operators depend on $l, m, p, \mu$ and $C_{\phi}$, but are independent of the concrete choice of the weight $\phi$.

Furthermore, the above results hold also for the family of spaces $W_{b, \phi}^{l, p}(\Omega)$.

Proof. As in the proof of Proposition 2.8, we restrict ourselves to consider only the case of a cylindrical domain $\Omega:=\mathbb{R} \times \omega$, one dimensional weights and the equivalent norms (2.20). Moreover, we will consider below only the case of spaces $W_{\phi}^{l, p}$ (the spaces $W_{b, \phi}^{l, p}$ can be considered analogously).

Indeed, let $u \in W_{\phi}^{l_{1}, p_{1}}(\Omega)$. Then, according to the classical Sobolev embedding theorem (see [25]), we have

$$
\|u\|_{W^{l_{2}, p_{2}\left(\Omega_{s}\right)}} \leq C\|u\|_{W^{l_{1}, p_{1}\left(\Omega_{s}\right)}}
$$

where the constant $C$ is independent of $s$. Taking the power $p_{2}$ of both sides of that inequality, we transform it to the following form (for simplicity, we consider only the case $\left.p_{2}<\infty\right)$

$$
\|u\|_{W^{l_{2}, p_{2}\left(\Omega_{s}\right)}}^{p_{2}} \leq C^{p_{2}}\|u\|_{W^{l_{1}, p_{1}\left(\Omega_{s}\right)}}^{p_{2}} \leq C_{1}\left(\int_{s \in \mathbb{R}} e^{-\alpha p_{1}|s-y|}\|u\|_{W^{l_{1}, p_{1}\left(\Omega_{y}\right)}}^{p_{1}} d y\right)^{p_{2} / p_{1}}
$$

where $\alpha>\mu$ is arbitrary and the constant $C_{1}$ is independent of $u$. Multiplying this relation by $\phi(s)^{p_{2}}$ integrating by $s \in \mathbb{R}$ and using inequality (2.12), we obtain

$$
\|u\|_{W_{\phi}^{l_{2}, p_{2}}(\Omega)}^{p_{2}} \leq C_{2}\|u\|_{W_{\phi}^{l_{1}, p_{1}}(\Omega)}^{p_{2}}
$$

which proves the first part of the proposition.

Let us verify the second assertion of the proposition. Indeed, the existence and boundedness of the trace operator $\Pi_{\partial \Omega}^{m}$ can be verified based on the analogous property for domains $\Omega_{s}$ exactly as before (so we leave it to the reader). Thus, we only need to construct the extension operator $\left[\Pi_{\partial \Omega}^{m}\right]^{-1}$. Indeed, let $U:=\left\{u_{k}\right\}_{k=0}^{m} \in$ $\otimes_{k=0}^{m} W_{\phi}^{l-k-1 / p, p}(\partial \Omega)$ be arbitrary. Using now the partition of unity (2.21), we construct the family $U_{s}:=\psi_{s} U=\left\{\psi_{s} u_{k}\right\}_{k=0}^{m}$. Then, since all of these functions vanish at the origins of the cylinder $\Omega_{s}$, there exists an extension operator $\left[\Pi_{\partial \Omega_{s}}^{m}\right]^{-1}$ for bounded domain $\Omega_{s}$ which maps $U_{s}$ to $W^{l, p}\left(\Omega_{s}\right)$ and its norm is independent of $U$ and $s$, see [25]. The required extension operator $\left[\Pi_{\partial \Omega}^{m}\right]^{-1}$ can be now constructed as follows:

$$
\left[\Pi_{\partial \Omega}^{m}\right]^{-1} U:=\int_{s \in \mathbb{R}}\left[\Pi_{\partial \Omega_{s}}^{m}\right]^{-1} U_{s} d s .
$$

Indeed, the fact that this operator is well defined, and the required uniform (with respect to $\phi)$ estimate for its norm as the map from $\otimes_{k=0}^{m} W_{\phi}^{l-k-1 / p, p}(\partial \Omega)$ to $W_{\phi}^{l, p}(\Omega)$, can be verified exactly as estimate (2.27) for the function (2.25) from the proof of Proposition 2.8., Proposition 2.10 is proved.

Our next task is to formulate some trace theorems for classes of less smooth functions which are closely related to the theory of NS equations. To this end, we need the following definition.

Definition 2.11. Let $\Omega$ be a regular domain of $\mathbb{R}^{n}, \phi$ be a weight function of exponential growth rate $\mu$ and $1<p<\infty$. Let us define the space $E_{\phi}^{p}(\Omega)$ of 
vector-valued functions $u:=\left(u^{1}, \cdots, u^{n}\right) \in\left[D^{\prime}(\Omega)\right]^{n}$ by the following norm:

$$
\|u\|_{E_{\phi}^{p}(\Omega)}^{p}:=\|u\|_{\left[L_{\phi}^{p}(\Omega)\right]^{n}}^{p}+\|\operatorname{div} u\|_{L_{\phi}^{p}(\Omega)}^{p} .
$$

The spaces $E_{b, \phi}^{p}(\Omega)$ are defined analogously. Moreover, for every sufficiently smooth vector-valued function $u:=\left(u^{1}, \cdots, u^{n}\right)$, we denote by $l_{n} u:=\left.(\vec{u}, \vec{n})\right|_{\partial \Omega}$ the normal component of that function at the boundary.

Proposition 2.12. Let $\Omega$ be a regular domain and $\phi$ be a weight function of exponential growth rate $\mu$. Then the operator $l_{n}: E_{\phi}^{p}(\Omega) \rightarrow W_{\phi}^{-1 / p, p}(\partial \Omega)$ is well-defined and

$$
\left\|l_{n} u\right\|_{W_{\phi}^{-1 / p, p}(\partial \Omega)} \leq C\|u\|_{E_{\phi}^{p}(\Omega)}
$$

where the constant $C$ depends on $\mu$ and $C_{\phi}$, but is independent of the concrete choice of the weight function $\phi$. Moreover, the analogous result holds also for the spaces $E_{b, \phi}^{p}(\Omega)$.

Proof. As before, we verify estimate (2.35) only for the cylindrical domains. Indeed, let $u$ and $v_{s}$ be smooth functions in $\Omega_{s}$. Then, due to Green's formula,

$$
\left(l_{n} u, v\right)_{\partial \Omega_{s}}:=(\operatorname{div} u, v)_{\Omega_{s}}+\left(u, \nabla_{x} v\right)_{\Omega_{s}} .
$$

As usual, we see that the right-hand side of (2.36) is well-defined for all $u \in E^{p}\left(\Omega_{s}\right)$ and $v \in W^{1, q}\left(\Omega_{s}\right)$ where $1 / p+1 / q=1$. Moreover, due to the classical trace theorems, there exists an extension operator $\left[\Pi_{s}\right]^{-1}: W^{1-1 / q, q}\left(\partial \Omega_{s}\right) \rightarrow W^{1, q}\left(\Omega_{s}\right)$ whose norm is obviously independent of $s$. Thus, (2.36) shows that the functional $l_{n} u$ is well-defined and satisfies

$$
\left\|l_{n} u\right\|_{W^{-1 / p, p}\left(\partial \Omega_{s}\right)}=\left\|l_{n} u\right\|_{\left[W^{1-1 / q, q}\left(\partial \Omega_{s}\right)\right]^{*}} \leq C\|u\|_{E^{p}\left(\Omega_{s}\right)} .
$$

Multiplying this relation by $\phi(s)^{p}$ and integrating over $s \in \mathbb{R}$, we deduce (2.35) and finish the proof of the proposition. Here we have implicitly used that

$$
\left\|l_{n} u\right\|_{W^{-1 / p, p}((s, s+1) \times \partial \omega)} \leq\left\|l_{n} u\right\|_{W^{-1 / p, p}\left(\partial \Omega_{s}\right)} .
$$

The estimate for $E_{b, \phi}^{p}(\Omega)$ can be obtained analogously using the supremum instead of integral over $s \in \mathbb{R}$.

As we have already mentioned, estimates of Proposition 2.5 allow us to reduce the proofs of elliptic regularity in arbitrary weighted spaces to the particular case of special weights (2.4). The following evident proposition will be useful in order to reduce the case of these special weights to the classical unweighted case $\phi=1$.

PROPOSITION 2.13. Let $\Omega$ be a regular domain and let $\mathbb{T}_{\mu, x_{0}}$ be a multiplication operator by the weight $\varphi_{\mu, x_{0}}(x)$ (i.e. $\left.\left(\mathbb{T}_{\mu, x_{0}} u\right)(x):=\varphi_{\mu, x_{0}}(x) u(x)\right)$. Then, for every $l \in \mathbb{R}$ and $1 \leq p \leq \infty$, this operator realizes an isomorphism between the spaces $W_{\varphi_{\mu, x_{0}}}^{l, p}(\Omega)$ and $W^{l, p}(\Omega)$. Moreover,

$$
C^{-1}\|u\|_{W_{\varphi \mu, x_{0}}^{l, p}(\Omega)} \leq\left\|\mathbb{T}_{\mu, x_{0}} u\right\|_{W^{l, p}(\Omega)} \leq C\|u\|_{W_{\varphi_{\mu, x_{0}}}^{l, p}(\Omega)}
$$

where the constant $C$ depends on $l, p$ and $\mu$, but is independent of $u$ and $x_{0} \in \mathbb{R}^{n}$. 
Indeed, this estimate is an immediate corollary of inequalities (2.6) and Definition 2.7 of the corresponding weighted spaces.

We now formulate the weighted analogue of one standard interpolation inequality which is crucial for the theory of 2D Navier-Stokes equation.

Proposition 2.14. Let $\Omega:=\mathbb{R} \times(0,1)$ be a strip $\left(\left(x_{1}, x_{2}\right) \in \Omega\right)$ and let $\phi_{i}, i=1,2$ be weight functions of the exponential growth rate $\mu$. Then the following interpolation inequality holds:

$$
\|u\|_{L_{\left(\phi_{1} \phi_{2}\right)^{1 / 2}}^{4}(\Omega)} \leq C\|u\|_{L_{\phi_{1}}^{2}(\Omega)}^{1 / 2}\|u\|_{W_{\phi_{2}}^{1,2}(\Omega)}^{1 / 2}
$$

where the constant $C$ depends on $C_{\phi_{i}}$ and $\mu$, but is independent of the concrete choice of weights $\phi_{i}$. Moreover, the analogous estimate holds for the spaces $W_{b, \phi}^{l, 2}(\Omega)$ as well.

Proof. Indeed, due to the interpolation inequality, we have

$$
\|u\|_{L^{4}\left(\Omega_{s}\right)}^{4} \leq\|u\|_{L^{2}\left(\Omega_{s}\right)}^{2}\|u\|_{W^{1,2}\left(\Omega_{s}\right)}^{2}
$$

where the constant $C$ is independent of $s$, see e.g. [16]. We transform this inequality as follows:

$$
\begin{aligned}
\|u\|_{L^{4}\left(\Omega_{s}\right)}^{4} & \leq C\left(\|u\|_{L^{2}\left(\Omega_{s}\right)}\|u\|_{W^{1,2}\left(\Omega_{s}\right)}\right)^{2} \\
& \leq C_{1}\left(\int_{s \in \mathbb{R}} e^{-\alpha|s-y|}\|u\|_{L^{2}\left(\Omega_{y} \cup \Omega_{y+1}\right)}\|u\|_{W^{1,2}\left(\Omega_{y} \cup \Omega_{y+1}\right)} d y\right)^{2} .
\end{aligned}
$$

Multiplying this relation by $\phi_{1}(s)^{2} \phi_{2}(s)^{2}$ and using estimate (2.12) and the Hölder inequality, we obtain

$$
\begin{aligned}
\|u\|_{L_{\left(\phi_{1} \phi_{2}\right)^{1 / 2}}^{4}(\Omega)}^{4} & \leq C_{2}\left(\int_{s \in \mathbb{R}} \phi_{1}(s) \phi_{2}(s)\|u\|_{L^{2}\left(\Omega_{s} \cup \Omega_{s+1}\right)}\|u\|_{W^{1,2}\left(\Omega_{s} \cup \Omega_{s+1}\right)} d s\right)^{2} \\
& \leq C_{2} \int_{s \in \mathbb{R}} \phi_{1}(s)^{2}\|u\|_{L^{2}\left(\Omega_{s} \cup \Omega_{s+1}\right)}^{2} d s \cdot \int_{s \in \mathbb{R}} \phi_{2}(s)^{2}\|u\|_{W^{1,2}\left(\Omega_{s} \cup \Omega_{s+1}\right)}^{2} d s \\
& \leq C_{3} \int_{s \in \mathbb{R}} \phi_{1}(s)^{2}\|u\|_{L^{2}\left(\Omega_{s}\right)}^{2} d s \cdot \int_{s \in \mathbb{R}} \phi_{2}(s)^{2}\|u\|_{W^{1,2}\left(\Omega_{s}\right)}^{2} d s
\end{aligned}
$$

which implies (2.40). The case of spaces $W_{b, \phi}^{l, 2}$ can be considered analogously. Proposition 2.14 is proven.

REMARK 2.15. The proof of Proposition 2.14 shows a general way of proving the weighted analogue of various interpolation inequalities. The most important for us here is the fact that the constants in these inequalities will depend only on the exponential growth rate $\mu$ and on the constants $C_{\phi}$ and will be independent of the concrete choice of the weights.

We conclude by formulating some useful results on the weighted and local topologies on bounded sets of $W_{b}^{l, p}(\Omega)$.

Proposition 2.16. Let $\Omega$ be a bounded domain $l \in \mathbb{R}$ and $p \in[1, \infty]$ and let $\mathbb{B}$ be a bounded subset of $W_{b}^{l, p}(\Omega)$. Then, for every weight function $\phi$ of exponential growth rate $\mu$ satisfying

$$
\|\phi\|_{L^{p}\left(\mathbb{R}^{n}\right)}<\infty
$$


the set $\mathbb{B}$ belongs to $W_{\phi}^{l, p}(\Omega)$ and the topology generated on $\mathbb{B}$ by this embedding is independent of the weight $\phi$ and coincides with the local topology on $\mathbb{B}$ generated by embedding to $W_{\text {loc }}^{l, p}(\bar{\Omega})$.

Proof. Indeed, due to (2.43), we have

$$
\|u\|_{W_{\phi}^{l, p}(\Omega)}^{p}=\int_{x_{0} \in \Omega} \phi^{p}\left(x_{0}\right)\|u\|_{W^{l, p}\left(\Omega \cap B_{x_{0}}^{1}\right)}^{p} d x_{0} \leq\|\phi\|_{L^{p}\left(\mathbb{R}^{n}\right)}^{p}\|u\|_{W_{b}^{l, p}(\Omega)}^{p}
$$

which shows that $W_{b}^{l, p}(\Omega) \subset W_{\phi}^{l, p}(\Omega)$. Suppose now the sequence $u_{n} \rightarrow u$ in $W_{l o c}^{l, p}(\bar{\Omega})$. This means that, for every $x_{0} \in \Omega$ and every $R \in \mathbb{R}_{+}$,

$$
\lim _{n \rightarrow \infty}\left\|u_{n}-u\right\|_{W^{l, p}\left(\Omega \cap B_{x_{0}}^{R}\right)}=0
$$

Let also $u_{n}, u \in \mathbb{B}$ and $\phi$ be an integrable (in the sense of (2.43)) weight. Then, since the set $\mathbb{B}$ is assumed to be bounded in $W_{b}^{l, p}(\Omega)$,

$$
\lim _{R \rightarrow \infty}\left\|u_{n}\right\|_{W_{\phi}^{l, p}\left(\Omega \backslash B_{0}^{R}\right)}=0
$$

uniformly with respect to $n \in \mathbb{N}$. Assertions (2.44) and (2.45) imply in a standard way that $u_{n} \rightarrow u$ in $W_{\phi}^{l, p}(\Omega)$. Since the embedding $W_{\phi}^{l, p}(\Omega) \subset W_{l o c}^{l, p}(\bar{\Omega})$ is obvious, then Proposition 2.16 is proven.

3. Elliptic regularity in weighted spaces. In this section, we recall some standard elliptic regularity results in weighted Sobolev spaces which are necessary to deal with the Navier-Stokes equations in unbounded domains. For simplicity, we restrict ourselves to consider only the case of a strip $\Omega:=\mathbb{R} \times(-1,1)\left(x:=\left(x_{1}, x_{2}\right) \in \Omega\right)$ although some of the results of this section remain true for general regular domains; see $[9-10,27-30]$ for details. We start with the weighted regularity estimate for the Laplacian with Dirichlet boundary conditions.

Proposition 3.1. Let us consider the following Dirichlet problem in a strip $\Omega$ :

$$
\Delta_{x} u=h,\left.\quad u\right|_{\partial \Omega}=0 .
$$

Then, for every $1<p<\infty$ and $l=-1,0,1$, there exists positive $\mu_{0}=\mu_{0}(p)$ such that, for every weight function $\phi$ with sufficiently small exponential growth rate $\mu\left(\mu \leq \mu_{0}\right)$ and every $h \in W_{\phi}^{l, p}(\Omega)$, equation (2.1) possesses a unique solution $u \in W_{\phi}^{l+2, p}(\Omega)$ and the following estimate holds:

$$
\|u\|_{W_{\phi}^{l+2, p}(\Omega)} \leq C\|h\|_{W_{\phi}^{l, p}(\Omega)}
$$

where the constant $C$ depends on $C_{\phi}$, but is independent of the concrete choice of the weight $\phi$. Moreover, the analogous estimate holds also for the spaces $W_{b, \phi}^{l, p}(\Omega)$.

Proof. We restrict ourselves to verify a priori estimate (3.2) only (the existence and uniqueness of a solution can be then verified in a standard way, see e.g. [9], [10]).

As we have already mentioned, due to estimates (2.12) and (2.13), it is sufficient to verify estimate (3.2) only for the special class of weights $\varphi_{\mu_{0}, x_{0}}(x)$ introduced in (2.4). Indeed, if we have estimate (3.2) for such weights with the constant $C$ independent of 
$x_{0}$, then we obviously have the following estimate:

$$
\begin{aligned}
\|u\|_{W^{l+2, p}\left(\Omega_{s}\right)}^{p} & \leq C_{\mu_{0}}\|u\|_{W_{\varphi \mu_{0}, s}^{l+2, p}(\Omega)}^{p} \leq C_{1}\|h\|_{W_{\varphi_{\mu_{0}, s}}^{l, p}(\Omega)}^{p} \\
& \leq C_{2} \int_{y \in \mathbb{R}} e^{-p \mu_{0}|s-y|}\|h\|_{W^{l, p}\left(\Omega_{y}\right)}^{p} d y
\end{aligned}
$$

where the constant $C_{2}$ is also independent of $s \in \mathbb{R}$. Multiplying now estimate (3.3) by $\phi(s)^{p}$ (where $\phi$ is a weight function with exponential growth rate $\mu<\mu_{0}$ ), integrating over $s \in \mathbb{R}$ and using estimate (2.12), we infer the required estimate (2.2). Analogously, estimate (3.2) for the spaces $W_{b, \phi}^{l, p}$ can be obtained by multiplication (3.3) by $\phi(s)^{p}$, taking the supremum over $s \in \mathbb{R}$ and using estimate (2.13).

Thus, it only remains to verify (3.2) for the special weights $\varphi_{\mu_{0}, s}$ with a sufficiently small positive $\mu_{0}$ and every $s \in \mathbb{R}$. In turns, due to Proposition 2.13 and estimates (2.6), the case of special weights $\varphi_{\mu_{0}, s}$ can be easily reduced to the unweighted case $\phi \equiv 1$. Indeed, the function $u \in W_{\varphi_{\mu_{0}}}^{l+2, p}(\Omega)$ solves (3.3) if and only if the function $v:=\varphi_{\mu_{0}, s} u \in W^{l+2, p}(\Omega)$ solves the following perturbed version of problem (3.2):

$$
\Delta_{x} v=\varphi_{\mu_{0}, s} h-\varphi_{\mu_{0}, s} \varphi_{-\mu_{0}, s}^{\prime \prime} v-2 \phi_{-\mu_{0}, s}^{\prime} \phi_{\mu_{0}, s} \partial_{x_{1}} v:=\mathbb{T}_{\mu_{0}, s} h+h_{\mu_{0}}(v),\left.v\right|_{\partial \Omega}=0 .
$$

We recall that, due to (2.6),

$$
\left\|h_{\mu_{0}}(v)\right\|_{W^{l, p}(\Omega)} \leq C \mu_{0}\|v\|_{W^{l+2, p}(\Omega)}
$$

where the constant $C$ is independent of $s$ and $\mu_{0}$. Thus, if estimate (3.2) for $\phi \equiv 1$ is known, then applying it to equation (3.4) and using (3.5), we infer

$$
\left\|\mathbb{T}_{\mu_{0}, s} u\right\|_{W^{l+2, p}(\Omega)} \leq C\left(\left\|\mathbb{T}_{\mu_{0}, s} h\right\|_{W^{l, p}(\Omega)}+\mu_{0}\|v\|_{W^{l+2, p}(\Omega)}\right)
$$

with the constant $C$ independent of $\mu_{0}$ and $s$. Fixing $\mu_{0}$ now to be small enough that $C \mu_{0}<1 / 2$, we deduce from the last estimate that

$$
\|v\|_{W^{l+2, p}(\Omega)} \leq 2 C\left\|\mathbb{T}_{\mu_{0}, s} h\right\|_{W^{l, p}(\Omega)}
$$

which together with Proposition 2.13 implies estimate (3.2) for special weights $\varphi_{\mu_{0}, s}$.

Thus, we have reduced the verifying of the regularity estimate (3.2) in weighted spaces to the unweighted case $\phi \equiv 1$. It only remains to note that (3.2) with $\phi \equiv 1$ is a classical $L^{p}$-regularity estimate for the solutions of the Laplace operator, see e.g. [16], [25]. Proposition 3.1 is proved.

REMARK 3.2. Surely, regularity estimate (3.2) holds not only for $l=-1,0,1$, but we will need it in the sequel only for these values of $l$. We also note that estimate (3.2) holds for the unweighted space since the spectrum of the Laplacian in a strip with Dirichlet boundary conditions is strictly negative.

The next proposition gives the elliptic regularity for the biLaplace operator in a strip $\Omega$.

Proposition 3.3. Let $\Omega$ be a strip and let us consider the following boundary value problem in $\Omega$ :

$$
\begin{aligned}
\Delta_{x}^{2} u & =h, \\
\left.u\right|_{\partial \Omega} & =h_{0},\left.\quad \partial_{n} u\right|_{\partial \Omega}=h_{1} .
\end{aligned}
$$


Then, for every $1<p<\infty$ and $l=0,1,2$, there exists $\mu_{0}=\mu_{0}(p)$ such that, for every weight function of a sufficiently small exponential growth rate $\mu\left(\mu \leq \mu_{0}\right)$ and every

$$
\left(h, h_{0}, h_{1}\right) \in W_{\phi}^{l-2, p}(\Omega) \times W_{\phi}^{l+2-1 / p, p}(\partial \Omega) \times W_{\phi}^{l+1-1 / p, p}(\partial \Omega)
$$

problem (3.7) has a unique solution $u \in W_{\phi}^{l+2, p}(\Omega)$ and the following estimate holds:

$$
\|u\|_{W_{\phi}^{l+2, p}(\Omega)} \leq C\left(\|h\|_{W_{\phi}^{l-2, p}(\Omega)}+\left\|h_{0}\right\|_{W_{\phi}^{l+2-1 / p, p}(\partial \Omega)}+\left\|h_{1}\right\|_{W_{\phi}^{l+1-1 / p, p}(\partial \Omega)}\right)
$$

where the constant $C$ depends on $C_{\phi}$, but is independent of the concrete choice of weight function $\phi$. Moreover, the analogous result holds for the spaces $W_{b, \phi}^{l, p}$ as well.

Proof. We first note that, due to the embedding (trace) theorem for weighted spaces formulated in Proposition 2.10, we can assume without loss of generality that $h_{0}=h_{1}=0$. Moreover, arguing as in the proof of Proposition 3.1, we can reduce the derivation of estimate (3.8) to the unweighted case $\phi \equiv 1$. After that it only remains to note that the spectrum of the biLaplacian $-\Delta^{2}$ in a strip $\Omega$ with homogeneous Dirichlet boundary conditions $\left.u\right|_{\partial \Omega}=\left.\partial_{n} u\right|_{\partial \Omega}=0$ is strictly negative. Thus, for the unweighted case (3.8) is just a classical $L^{p}$-regularity result for the 4 th order elliptic operator $-\Delta_{x}^{2}$, see [25]. Proposition 3.3 is proved.

We are now going to consider the Newmann-type boundary value problems for the Laplacian in a strip $\Omega$. The main difficulty here is the fact that, in contrast to the Dirichlet problems considered above, the Newmann problem for the Laplacian has an essential spectrum at $\lambda=0$, which makes the situation much more delicate. We however start with the regularized Newmann-type problem where the spectrum remains strictly negative.

Proposition 3.4. Let $\Omega$ be a strip and let us consider the following boundary value problem in $\Omega$ :

$$
\Delta_{x} u-u=0,\left.\quad \partial_{n} u\right|_{\partial \Omega}=h_{0} .
$$

Then, for every $1<p<\infty$ and $l=0,1,2$, there exists $\mu_{0}=\mu_{0}(p)$ such that, for every weight function of sufficiently small exponential growth rate $\mu\left(\mu \leq \mu_{0}\right)$ and every $h_{0} \in W_{\phi}^{l-1 / p, p}(\partial \Omega)$, problem (3.9) has a unique solution $u \in W_{\phi}^{l+1, p}(\Omega)$ and the following estimate holds:

$$
\|u\|_{W_{\phi}^{l+1, p}(\Omega)} \leq C\left\|h_{0}\right\|_{W_{\phi}^{l-1 / p, p}(\partial \Omega)}
$$

where the constant $C$ depends on $C_{\phi}$, but is independent of the concrete choice of weight function $\phi$. Moreover, the analogous result holds for the spaces $W_{b, \phi}^{l, p}$ as well.

Proof. Indeed, in the case $l=1,2$ estimate (3.10) can be verified exactly as in Propositions 3.1 and 3.3 (by reducing to the homogeneous and unweighted case), so we leave it to the reader. In the case $l=0$ the situation is slightly more delicate since we do not formulate the extension theorem for the space $W_{\phi}^{-1 / p, p}(\Omega)$ in Proposition 2.10 and, consequently, we need to work with the nonhomogeneous boundary value problem. Nevertheless, the reduction to the unweighted case based on introducing the 
function $v:=\varphi_{\mu_{0}, s} u$ works in this case as well. Indeed, this function obviously satisfies

$$
\Delta_{x} v-v=h_{\mu_{0}}(v),\left.\quad \partial_{n} v\right|_{\partial \Omega}:=\mathbb{T}_{\mu_{0}, s} h_{0}
$$

and

$$
\left\|h_{\mu_{0}}(v)\right\|_{L^{p}(\Omega)} \leq C \mu_{0}\|v\|_{W^{1, p}(\Omega)} .
$$

Thus, we can split the solution $v$ of (3.11) as follows: $v=v_{1}+v_{2}$ where $v_{1}$ solves the homogeneous problem

$$
\Delta_{x} v_{1}-v_{1}=h_{\mu_{0}}(v),\left.\quad \partial_{n} v_{1}\right|_{\partial \Omega}=0
$$

and the remainder $v_{2}$ solves the analogue of (3.9) with $h_{0}$ replaced by $\mathbb{T}_{\mu_{0}, s} h_{0}$. We see also that the right-hand side of (3.11) belongs to $L^{p}(\Omega)$ and, consequently, due to the classical $L^{p}$-regularity, we have

$$
\left\|v_{1}\right\|_{W^{2, p}(\Omega)} \leq C\left\|h_{\mu_{0}}(v)\right\|_{L^{p}(\Omega)} \leq C_{1} \mu_{0}\|v\|_{W^{1, p}(\Omega)} .
$$

If we assume now that estimate (3.10) for the unweighted case $\phi=1$ and $l=0$ is known, then, due to (3.14), we obtain

$$
\|v\|_{W^{1, p}(\Omega)} \leq\left\|v_{1}\right\|_{W^{1, p}(\Omega)}+\left\|v_{2}\right\|_{W^{1, p}(\Omega)} \leq C\left\|\mathbb{T}_{\mu_{0}, s} h_{0}\right\|_{W^{-1 / p . p}(\partial \Omega)}+C \mu_{0}\|v\|_{W^{1, p}(\Omega)}
$$

which implies the estimate

$$
\|v\|_{W^{1, p}(\Omega)} \leq 2 C\left\|\mathbb{T}_{\mu_{0}, s} h_{0}\right\|_{W^{-1 / p, p}(\partial \Omega)}
$$

if $\mu_{0}$ is small. Thus, the case of general weight naturally reduces to the case of $\phi \equiv 1$ for $l=0$ as well. It remains to recall that, for $\phi \equiv 1$, estimate (3.10) is a classical $L^{p}$-regularity result for the Laplacian, see [25]. Proposition 3.4 is proved.

In order to treat the case of the Newmann problem without the regularizing term $-u$, we need to introduce the following averaging operator with respect to the variable $x_{2}\left(\left(x_{1}, x_{2}\right) \in \mathbb{R} \times(-1,1):=\Omega\right)$ :

$$
(\mathrm{S} u)\left(x_{1}\right):=\frac{1}{2} \int_{-1}^{1} u\left(x_{1}, s\right) d s .
$$

The next proposition gives the solvability of the Newmann problem for some natural closed subspace of the the space of external forces $h$.

Proposition 3.5. Let $\Omega$ be a strip and let us consider the following boundary value problem in $\Omega$ :

$$
\Delta_{x} u=h,\left.\quad \partial_{n} u\right|_{\partial \Omega}=0 .
$$

Then, for every $1<p<\infty$ and $l=0,1,2$, there exists $\mu_{0}=\mu_{0}(p)$ such that, for every weight function of a sufficiently small exponential growth rate $\mu\left(\mu \leq \mu_{0}\right)$ and every $h \in W_{\phi}^{l, p}(\Omega)$ satisfying

$$
\mathbb{S} h \equiv 0,
$$


problem (3.17) has a unique solution $u \in W_{\phi}^{l+2, p}(\Omega)$, $\mathbb{S} u \equiv 0$ and the following estimate holds:

$$
\|u\|_{W_{\phi}^{l+2, p}(\Omega)} \leq C\|h\|_{W_{\phi}^{l, p}(\Omega)}
$$

where the constant $C$ depends on $C_{\phi}$, but is independent of the concrete choice of weight function $\phi$. Moreover, the analogous result holds for the spaces $W_{b, \phi}^{l, p}$ as well.

Proof. We first note that the operator $\$$ commutes with the multiplication operator $\mathbb{T}_{\mu_{0}}$ and with the $x_{1}$-derivatives $\partial_{x_{1}}$. Thus, arguing exactly as before, we can reduce the proof of (3.18) to the unweighted case $\phi \equiv 1$. So, we will prove below (3.18) for the case $\phi \equiv 1$ only.

To this end, we first consider the case $p=2$. In that case we can multiply equation (3.17) by $u$ and obtain, after integration by parts,

$$
\left\|\nabla_{x} u\right\|_{L^{2}(\Omega)}^{2} \leq\|h\|_{L^{2}(\Omega)}\|u\|_{L^{2}(\Omega)} .
$$

Since we have assumed additionally that $\mathbb{S} u \equiv 0$, we have the Friedrich's inequality

$$
\|u\|_{W^{1,2}(\Omega)} \leq C\left\|\nabla_{x} u\right\|_{L^{2}(\Omega)}
$$

which together with (3.19) implies that

$$
\|u\|_{W^{1,2}(\Omega)} \leq C\|h\|_{L^{2}(\Omega)} .
$$

In order to prove estimate (3.18) for $p=2$ and $\phi \equiv 1$, we now use the following standard interior regularity estimate:

$$
\begin{aligned}
\|u\|_{W^{l+2,2}\left(\Omega_{s}\right)}^{2} & \leq C\left(\|u\|_{W^{1,2}\left(\Omega_{s-1} \cup \Omega_{s} \cup \Omega_{s+1}\right)}^{2}+\|h\|_{W^{1,2}\left(\Omega_{s}\right)}^{2}\right) \\
& \leq C_{1} \int_{y \in \Omega} e^{-\alpha|s-y|}\left(\|u\|_{W^{1,2}\left(\Omega_{y}\right)}^{2}+\|h\|_{W^{l, 2}\left(\Omega_{y}\right)}^{2}\right) d y .
\end{aligned}
$$

Integrating this estimate over $s \in \mathbb{R}$ and using (2.12) and (3.21), we deduce the unweighted estimate (3.18) for $p=2$. Thus, due to the trick with the multiplication operator $\mathbb{T}_{\mu_{0}, s}$, estimate (3.18) is verified for $p=2$ and all weights with sufficiently small exponential growth rate. Moreover, we have also the analogue of estimate (3.18) with $p=2$ for the spaces $W_{b, \phi}^{l, p}(\Omega)$.

Let us now consider the case $p \neq 2$. We first consider the case $p>2$ and will prove estimate (3.18) for the spaces $W_{b}^{l, p}(\Omega)$. Indeed, since $W_{b}^{l, p}(\Omega) \subset W_{b}^{l, 2}(\Omega)$, then we already have the estimate

$$
\|u\|_{W_{b}^{1,2}(\Omega)} \leq C\|h\|_{L_{b}^{2}(\Omega)} \leq C_{1}\|h\|_{L_{b}^{p}(\Omega)} .
$$

Using now the interior regularity estimate

$$
\begin{aligned}
\|u\|_{W^{l+2, p}\left(\Omega_{s}\right)} & \leq C\left(\|u\|_{W^{1,2}\left(\Omega_{s-1} \cup \Omega_{s} \cup \Omega_{s+1}\right)}+\|h\|_{W^{l, p}\left(\Omega_{s}\right)}\right) \\
& \leq C_{1} \sup _{y \in \mathbb{R}}\left\{e^{-\alpha|s-y|}\left(\|u\|_{W^{1,2}\left(\Omega_{y}\right)}+\|h\|_{W^{l, p}\left(\Omega_{y}\right)}\right)\right\},
\end{aligned}
$$


taking a supremum over $s \in \mathbb{R}$ from the both parts of that inequality and using (2.3) and (3.23), we finally obtain

$$
\|u\|_{W_{b}^{l+2, p}(\Omega)} \leq C\|h\|_{W_{b}^{l, p}(\Omega)} .
$$

Now let $1<p<2$. Then we split the solution $u$ of (3.17) as follows: $u=u_{1}+u_{2}$ where $u_{1}$ solves problem

$$
\Delta_{x} u_{1}-u_{1}=h,\left.\quad \partial_{n} u_{1}\right|_{\partial \Omega}=0
$$

and the remainder $u_{2}$ solves

$$
\Delta_{x} u_{2}=-u_{1},\left.\quad \partial_{n} u_{2}\right|_{\partial \Omega}=0 .
$$

We first note that, due to the $L^{p}$-regularity (see Proposition 3.4), for equation (3.25), we have

$$
\left\|u_{1}\right\|_{W_{b}^{l+2, p}(\Omega)} \leq C\|h\|_{W_{b}^{l, p}(\Omega)} .
$$

Moreover, applying the operator $\mathbb{S}$ to both sides of equation (3.25) and using that Sh $\equiv 0$, we have

$$
\left(\mathbb{S} u_{1}\right)^{\prime \prime}-\mathbb{S} u_{1} \equiv 0 \text { and, consequently, } \mathbb{S} u_{1} \equiv 0 .
$$

Furthermore, due to the embedding theorem (see Proposition 2.10), we have

$$
\left\|u_{1}\right\|_{W^{l, 2}(\Omega)} \leq C\left\|u_{1}\right\|_{W_{b}^{l+2, p}(\Omega)},
$$

for every $1<p<2$. Thus, we can apply estimate (3.23) for equation (3.26) which together with (3.27) gives estimate (3.24) for $1<p<2$ as well.

Thus, estimate (3.24) is verified for all $1<p<\infty$. Then, due to the above described trick with the multiplication operator $\mathbb{T}_{\mu_{0}, s}$, we deduce estimate (3.18) for the spaces $W_{b, \phi}^{l+2, p}(\Omega)$ for all weight functions of sufficiently small exponential growth rate.

So, it only remains to obtain it for the spaces $W_{\phi}^{l, p}(\Omega)$. To this end, we note that (3.18) for the spaces $W_{b, \varphi_{\mu_{0}, s}}^{l, p}(\Omega)$ implies, in particular, that

$$
\|u\|_{W^{l+2, p}\left(\Omega_{s}\right)}^{p} \leq C \sup _{y \in \mathbb{R}}\left\{e^{-\mu_{0} p|s-y|}\|h\|_{W^{l, p}\left(\Omega_{y}\right)}^{p}\right\} \leq C_{1} \int_{y \in \Omega} e^{-\mu_{0} p|s-y|}\|h\|_{W^{l, p}(\Omega)}^{p} d y .
$$

Multiplying (3.30) by $\phi(s)^{p}$, integrating over $s \in \mathbb{R}$ and using (3.12), we deduce finally estimate (3.18) and finish the proof of Proposition 3.5.

REMARK 3.6. As we see from the proof of Proposition 3.5, the weighted regularity estimates can be deduced not only from the unweighted estimates in $W^{l, p}(\Omega)$, but also from its analogies in the spaces $W_{b}^{l, p}(\Omega)$. The last scale of spaces is sometimes (e.g., in the proof of Proposition 3.5) more convenient, since, in contrast to spaces $L^{p}(\Omega)$, the spaces $L_{b}^{p}(\Omega)$ have usual (for bounded domains) embedding properties $\left(L_{b}^{p_{1}}(\Omega) \subset L_{b}^{p_{2}}(\Omega)\right.$, for $\left.p_{1} \geq p_{2}\right)$.

We now note that assumption $\mathbb{S} h \equiv 0$ in Proposition 3.5 is essential for the weighted estimate (3.18). Indeed, in general case $\mathbb{S} h \neq 0$, for the quantity 
$\mathbb{S} u=(\mathbb{S} u)\left(x_{1}\right)$ we have the following equation:

$$
(\mathbb{S} u)\left(x_{1}\right)^{\prime \prime}=(\mathbb{S} h)\left(x_{1}\right), \quad x_{1} \in \mathbb{R}
$$

whose solution $\mathbb{S} u$, obviously, does not possess any weighted regularity estimates for general $h$. Fortunately, for problems arising in the weighted regularity theory for the Helmholtz operator, the function $\mathbb{S} h$ has a special structure which allows us to take one primitive of it remaining in weighted Sobolev classes. To be more precise, the following proposition holds.

Proposition 3.7. Let $\Omega$ be a strip and let us consider the following Newmann boundary value problem in $\Omega$ :

$$
\Delta_{x} u=0,\left.\quad \partial_{n} u\right|_{\partial \Omega}=l_{n} g
$$

where $g \in\left[L^{p}(\Omega)\right]^{2}$ is a divergent free vector field

$$
\operatorname{div} g \equiv 0 \text {. }
$$

Then, for every $1<p<\infty$ and $l=0,1,2$, there exists $\mu_{0}=\mu_{0}(p)$ such that, for every weight function of a sufficiently small exponential growth rate $\mu\left(\mu \leq \mu_{0}\right)$ and every $g \in W_{\phi}^{l, p}(\Omega)$ satisfying (3.33), problem (3.32) has a unique solution (up to adding a constant) satisfying $\nabla_{x} u \in W_{\phi}^{l, p}(\Omega)$, and

$$
(\mathbb{S} u)\left(x_{1}\right)^{\prime}=\left(\mathbb{S} g_{1}\right)\left(x_{1}\right), \quad x_{1} \in \mathbb{R}
$$

and the following estimate holds:

$$
\left\|\nabla_{x} u\right\|_{W_{\phi}^{l, p}(\Omega)} \leq C\|g\|_{W_{\phi}^{l, p}(\Omega)}
$$

where the constant $C$ depends on $C_{\phi}$, but is independent of the concrete choice of weight function $\phi$. Moreover, the analogous result holds for the spaces $W_{b, \phi}^{l, p}$ as well.

Proof. For simplicity, we deduce below only a priori estimate (3.35). The existence and uniqueness of a solution can be verified in a standard way (see also [4].

We first define an auxiliary function $v$ as a solution of the following problem:

$$
\Delta_{x} v-v=0,\left.\quad \partial_{n} v\right|_{\partial \Omega}=l_{n} g .
$$

Then, due to Propositions 3.4 and 2.12, we have

$$
\|v\|_{W_{\phi}^{l+1, p}(\Omega)} \leq C\left\|l_{n} g\right\|_{W_{\phi}^{l-1 / p, p}(\partial \Omega)} \leq C_{2}\|g\|_{W_{\phi}^{l, p}(\Omega)} .
$$

Moreover, applying the $x_{2}$-averaging operator $\mathbb{S}$ to equation (3.36), we have

$$
(\mathbb{S} v)\left(x_{1}\right)^{\prime \prime}-(\mathbb{S} v)\left(x_{1}\right)=-1 / 2\left(g_{2}\left(x_{1}, 1\right)-g_{2}\left(x_{1},-1\right)\right), \quad x_{1} \in \mathbb{R} .
$$

Furthermore, since the vector field $g$ is divergence free, we have

$$
1 / 2\left(g_{2}\left(x_{1}, 1\right)-g_{2}\left(x_{1},-1\right)\right)=\left(\mathbb{S}\left[\partial_{x_{2}} g_{2}\right]\right)\left(x_{1}\right)=-\left(\mathbb{S} g_{1}\right)\left(x_{1}\right)^{\prime}
$$

and, consequently,

$$
(\mathbb{S} v)\left(x_{1}\right)^{\prime \prime}-(\mathbb{S} v)\left(x_{1}\right)=\left(\mathbb{S} g_{1}\right)\left(x_{1}\right)^{\prime}
$$


Let us consider now the remainder $w:=u-v$ which obviously satisfies the following equation:

$$
\Delta_{x} w=-v,\left.\quad \partial_{n} w\right|_{\partial \Omega}=0 .
$$

Then, according to Proposition 3.5, the function $\bar{w}:=w-\mathbb{S} w$ satisfies the following estimate:

$$
\|\bar{w}\|_{W_{\phi}^{l+1, p}(\Omega)} \leq C\|\bar{v}\|_{W_{\phi}^{l, p}(\Omega)} \leq C_{1}\|g\|_{W_{\phi}^{l, p}(\Omega)} .
$$

So, it only remains to consider the equation for $\mathbb{S} w$, i.e.

$$
(\mathbb{S} w)\left(x_{1}\right)^{\prime \prime}=-(\mathbb{S} v)\left(x_{1}\right)
$$

which together with (3.39) gives

$$
(\mathbb{S} u)\left(x_{1}\right)^{\prime \prime}=(\mathbb{S} g)\left(x_{1}\right)^{\prime} .
$$

This relation shows that we can indeed take one primitive and satisfy condition (3.34). It only remains to note that the function $(\mathbb{S} u)\left(x_{1}\right)$ is independent of $x_{2}$ and, consequently,

$$
\nabla_{x} u=\nabla_{x} \bar{u}+\left((\mathbb{S} u)^{\prime}, 0\right) .
$$

Thus, estimates (3.37), (3.41) together with the obvious fact that

$$
\|S g\|_{W_{\phi}^{l, p}(\mathbb{R})} \leq C\|g\|_{W_{\phi}^{l, p}(\Omega)}
$$

yield (3.35) and finish the proof of Proposition 3.7.

4. Weighted spaces and the Helmholtz projector. In this Section, we discuss the weighted analogue of the classical Helmholtz decomposition of the space $\left[L^{2}(\Omega)\right]^{2}$ to divergent free and gradient vector fields, which is necessary for excluding the pressure from Navier-Stokes equations. To this end, we first need to define the corresponding spaces of divergent free vector fields.

Definition 4.1. Let $\Omega$ be a strip. Then, for every $l \geq 0,1<p<\infty$ and every weight function $\phi$ of exponential growth rate, we define the following space of divergent free vector fields:

$$
\mathcal{H}_{\phi}^{l, p}(\Omega):=\left\{v \in\left[W_{\phi}^{l, p}(\Omega)\right]^{2}, \operatorname{div} u v \equiv 0,\left.\quad l_{n} v\right|_{\partial \Omega}=0, \quad S v_{1} \equiv 0\right\}
$$

which is considered as a closed subspace of $W_{\phi}^{l, p}(\Omega)$ and endowed by the norm induced by this embedding. Here the normal component $l_{n} v$ of the trace on the boundary is well-defined due to Proposition 2.12 and the $x_{2}$-averaging operator $\mathbb{S}$ is defined by (3.16). The spaces $\mathcal{H}_{b, \phi}^{l, p}(\Omega)$ can be defined analogously. Moreover, for simplicity, we will henceforth write $\mathcal{H}_{\phi}^{p}(\Omega)$ and $\mathcal{H}_{b, \phi}^{p}(\Omega)$ instead of $\mathcal{H}_{\phi}^{0, p}(\Omega)$ and $\mathcal{H}_{b, \phi}^{0, p}(\Omega)$ respectively.

We also define the space $\mathcal{V}_{\phi}^{p}(\Omega)$ as follows:

$$
\mathcal{V}_{\phi}^{p}(\Omega):=\left\{v \in \mathcal{H}_{\phi}^{1, p}(\Omega),\left.\quad v\right|_{\partial \Omega}=0\right\}
$$

and the analogous space $\mathcal{V}_{b, \phi}^{p}(\Omega)$. 
The following natural proposition clarifies the additional conditions $\left.l_{n} v\right|_{\partial \Omega}=0$ and $\mathbb{S} v_{1} \equiv 0$ in formula (4.1).

PROPOSITION 4.1. Let $\Omega$ be a strip and $\phi$ be a weight function of exponential growth rate $\mu$ and $1<p<\infty$. Then the space $\mathcal{H}^{p}(\Omega)$ coincides with the closure of all divergent free vector fields $v \in[\mathcal{D}(\Omega)]^{2}$ in the topology of $\left[L_{\phi}^{p}(\Omega)\right]^{2}$ :

$$
\mathcal{H}_{\phi}^{p}(\Omega)=\left[v \in[\mathcal{D}(\Omega)]^{2}, \quad \operatorname{div} v=0\right]_{\left[L_{\phi}^{p}(\Omega)\right]^{2}}
$$

where $[\cdot]_{V}$ denotes the closure in the topology of the space $V$.

Proof. Indeed, let $v$ be a divergent free vector field from $[D(\Omega)]^{2}$. Then, obviously, $\left.l_{n} v\right|_{\partial \Omega}=0$. Moreover, integrating the relation $\partial_{x_{1}} v_{1}=-\partial_{x_{2}} v_{2}$, we infer that $\mathbb{S} v_{1} \equiv$ constant $=0$ (since $v_{1}$ has a finite support). Since all these properties preserve under the closure (see Proposition 2.12), then the right-hand side of (4.2) is a subset of the left one.

Thus, it only remains to approximate every function from $u \in \mathcal{H}_{\phi}^{p}(\Omega)$ by divergent free vector fields belonging to $[\mathcal{D}(\Omega)]^{2}$. In order to do so, it is natural to use the stream function $\Phi$ of a divergent free vector field $u$ :

$$
u_{1}=\partial_{x_{2}} \Phi, \quad u_{2}=-\partial_{x_{1}} \Phi
$$

which can be defined by the following natural formula:

$$
\Phi\left(x_{1}, x_{2}\right):=\int_{-1}^{x_{2}} u_{1}\left(x_{1}, \theta\right) d \theta .
$$

Indeed, obviously, $\Phi \in L_{\phi}^{p}(\Omega)$ and

$$
\|\Phi\|_{L_{\phi}^{p}(\Omega)} \leq C\left\|u_{1}\right\|_{L_{\phi}^{p}(\Omega)} .
$$

Moreover, $\partial_{x_{2}} \Phi=u_{1}$ and

$$
\partial_{x_{1}} \Phi\left(x_{1}, x_{2}\right)=\int_{-1}^{x_{2}} \partial_{x_{1}} u_{1}\left(x_{1}, s\right) d s=-\int_{-1}^{x_{2}} \partial_{x_{2}} u_{2}\left(x_{1}, s\right) d s=-u_{2}\left(x_{1}, x_{2}\right)
$$

(here we have implicitly used that $\operatorname{div} u \equiv 0$ and $\left.u_{2}\left(x_{1},-1\right) \equiv u_{2}\left(x_{1}, 1\right)=0\right)$. Thus, the function $\Phi$ indeed satisfies relations (4.3) and, consequently, $\Phi \in W_{\phi}^{1, p}(\Omega)$ and

$$
\|\Phi\|_{W_{\phi}^{l, p}(\Omega)} \leq C\|u\|_{L_{\phi}^{p}(\Omega)} .
$$

Finally, since $\mathbb{S} u_{1} \equiv 0$, then $\Phi\left(x_{1},-1\right)=\Phi\left(x_{1}, 1\right) \equiv 0$ and, consequently, $\Phi \in W_{0, \phi}^{1, p}$. It only remains to note that every function $\Phi$ from $W_{0, \phi}^{1, p}(\Omega)$ can be approximated by the smooth functions with compact supports. Then, formula (4.3) gives the required approximation of the vector field $u$. Proposition (4.2) is proved.

REMARK 4.3. In Proposition 4.2, we have actually proved that formulae (4.3) and (4.4) realize the isomorphism between spaces $W_{0, \phi}^{1, p}(\Omega)$ and $\mathcal{H}_{\phi}^{p}(\Omega)$ (and also between the spaces $W_{0, b, \phi}^{1, p}(\Omega)$ and $\left.\mathcal{H}_{b, \phi}^{p}(\Omega)\right)$. Moreover, it is not difficult to see that this map also realizes an isomorphism between $\mathcal{V}_{\phi}^{p}(\Omega)$ and $W_{0, \phi}^{2, p}(\Omega)$ (and between $\mathcal{V}_{b, \phi}^{p}(\Omega)$ and $W_{0, b, \phi}^{2, p}(\Omega)$ as well). 
As usual, we define the operator $\Pi:\left[L^{2}(\Omega)\right]^{2} \rightarrow \mathcal{H}^{2}(\Omega)$ as an orthoprojector to the divergent free vector fields. Then, as known (see e.g. [23] or [24]), every vector field $u \in\left[L^{2}(\Omega)\right]^{2}$ can be split in a unique way as a sum of a divergent free vector field $v \in \mathcal{H}^{2}(\Omega)$ and a potential one $\nabla_{x} p \in\left[L^{2}(\Omega)\right]^{2}$ for the appropriate $p \in H_{\text {loc }}^{1}(\bar{\Omega})$ :

$$
u=v+\nabla_{x} p, \quad \operatorname{div} v=0, \quad v:=\Pi u .
$$

The next theorem shows that the analogous splitting holds in weighted spaces as well.

THEOREM 4.4. Let $\Omega$ be a strip and let $\Pi$ be the orthoprojector defined above. Then, for every $1<p<\infty$ and $l=0,1,2$, there exists a sufficiently small positive $\mu_{0}$ such that, for every weight function with exponential growth rate $\mu \leq \mu_{0}$, this projector can be uniquely extended by continuity to a bounded operator from $\left[\bar{W}_{\phi}^{l, p}(\Omega)\right]^{2}$ to $\mathcal{H}_{\phi}^{l, p}(\Omega)$ and the following estimate holds:

$$
\|\Pi u\|_{\mathcal{H}_{\phi}^{l, p}(\Omega)} \leq C\|u\|_{\left[W_{\phi}^{l, p}(\Omega)\right]^{2}}
$$

where the constant $C$ depends only on $p$, land $C_{\phi}$, but is independent of the concrete choice of the weight $\phi$. Thus, for every $u \in\left[W_{\phi}^{l, p}(\Omega)\right]^{2}$ there exists a unique decomposition in the form of (4.7) with $v \in \mathcal{H}_{\phi}^{l, p}(\Omega)$ and $p \in W_{\text {loc }}^{l+1, p}(\bar{\Omega})$. In this formula $v=\Pi u$. Moreover, the analogous result holds also for the spaces $W_{b, \phi}^{l, p}$.

Proof. Indeed, assume that (4.7) is satisfied for some functions $u, v$ and $p$. Let us also introduce the stream function $\Phi \in W_{0, \phi}^{1, p}(\Omega)$ associated with the divergent free vector field $v$ via (4.4). Then,

$$
\Delta_{x} \Phi=\partial_{x_{2}} v_{1}-\partial_{x_{1}} v_{2}=\partial_{x_{2}} u_{1}-\partial_{x_{1}} u_{2},\left.\quad \Phi\right|_{\partial \Omega}=0 .
$$

Thus, due to Proposition 3.1, there exists a unique $\Phi \in W_{0, \phi}^{l+1, p}(\Omega)$ which satisfies (4.9). Moreover, the following estimate holds:

$$
\|\Phi\|_{W_{\phi}^{l+1, p}(\Omega)} \leq C\left\|\partial_{x_{2}} u_{1}-\partial_{x_{1}} u_{2}\right\|_{W_{\phi}^{l-1, p}(\Omega)} \leq C_{1}\|u\|_{W_{\phi}^{l, p}(\Omega)}
$$

for every weight function of a sufficiently small exponential growth rate. Since the vector field $v$ can be found via $\Phi$ by (4.3), then (4.10) shows that the projector $\Pi$ is really well defined for every $u \in\left[W_{\phi}^{l, p}(\Omega)\right]^{2}$ and satisfies estimate (4.8). So, we only need to verify decomposition (4.7).

Indeed, let $u \in\left[W_{\phi}^{l, p}(\Omega)\right]^{2}$ be arbitrary and let $v:=\Pi u$ and $w:=u-v$. Then, obviously, $w \in\left[W_{l o c}^{l, p}(\bar{\Omega})\right]^{2}$ and satisfies (in the sense of distributions) the following relation:

$$
\partial_{x_{2}} w_{1}=\partial_{x_{1}} w_{2}
$$

Consequently, since $\Omega$ is simply connected, there exists a potential $p \in W_{l o c}^{l+1, p}(\bar{\Omega})$ such that $w=\nabla_{x} p$ (this potential is obviously defined up to a constant, see [23]). Thus splitting (4.7) is also verified and Theorem 4.4 is proved.

REMARK 4.5. There exists a more general (and a slightly more complicated) way to find the potential $p$ from relation (4.7). Indeed, taking a divergence from the both 
parts of (4.7), we get

$$
\Delta_{x} p=\operatorname{div} u
$$

and using the fact that $\left.l_{n} v\right|_{\partial \Omega}=0$, we infer the boundary condition for $p$ :

$$
\left.\partial_{n} p\right|_{\partial \Omega}=\left.l_{n} u\right|_{\partial \Omega}
$$

We note however that the right-hand side of (4.13) is ill-posed for general $u \in\left[L^{p}(\Omega)\right]^{2}$. In order to overcome this difficulty, we introduce an auxiliary function $p_{1}$ which solves

$$
\Delta_{x} p_{1}=\operatorname{div} u,\left.\quad p_{1}\right|_{\partial \Omega}=0
$$

and then the remainder $\bar{p}:=p-p_{1}$ solves

$$
\Delta_{x} \bar{p}=0,\left.\quad \partial_{n} \bar{p}\right|_{\partial \Omega}=\left.l_{n}\left(h-\nabla_{x} p\right)\right|_{\partial \Omega} .
$$

We now note that div $\left(h-\nabla_{x} p_{1}\right)=0$ and, consequently, due to Proposition 2.12 , the trace $l_{n}\left(h-\nabla_{x} p_{1}\right)$ on the boundary is well-defined and we can apply Proposition 3.7 which gives a unique solvability (up to a constant) of (4.15) and estimate (3.35) for the gradient of $\bar{p}$. It remains to note that condition (3.34) now reads

$$
\partial_{x_{1}} \mathbb{S} \bar{p}=\mathbb{S} u_{1}-\partial_{x_{1}} \mathbb{S} p_{1} \text { and, thus } \mathbb{S} \partial_{x_{1}} p=\mathbb{S} u_{1}
$$

which shows that $p$ is indeed correctly defined $\left(\mathbb{S} v_{1}=\mathbb{S} u_{1}-\mathbb{S} \partial_{x_{1}} p=0\right.$, div $v=0$ and $\left.l_{n} v=0\right)$.

The advantage of this method is that, in contrast to the scheme used in the proof of Theorem 4.4, it works not only for 2D strips, but also for 3D cylindrical domains, see [4] and [32] for the details.

COROLlaRY 4.6. Let the assumptions of Theorem 4.4 hold and let $v \in \mathcal{H}_{\phi}^{p}(\Omega)$. Then, for every potential vector field $w=\nabla_{x}$ p such that $w \in\left[L_{\phi^{-1}}^{q}(\Omega)\right]^{2}$, we have

$$
(v, w)_{\left[L^{2}(\Omega)\right]^{2}}=0 .
$$

Indeed, according to Proposition 4.2, the function $v$ can be approximated (in the metric of $L_{\phi}^{p}(\Omega)$ ) by a sequence of smooth divergent free vector fields with a compact support. Since for such vector fields (4.16) is obvious, then passing to the limit, we obtain (4.16) for all $v \in \mathcal{H}_{\phi}^{p}(\Omega)$.

The next proposition gives the estimate for the weighted norms of the commutator of $\Pi$ and the multiplication operator $\mathbb{T}_{\mu, x_{0}}$ introduced in Proposition 2.13.

Proposition 4.7. Let $\Omega$ be a strip, $1<p<\infty, l=0,1,2$ and $\mathbb{T}_{\mu, x_{0}}$ a multiplication by the special weight $\varphi_{\mu, x_{0}}\left(x_{1}\right)$. Then there exists $\mu_{0}=\mu_{0}(p)>0$ such that, for every weight function of exponential growth rate $\varepsilon \leq \mu_{0}$, every $\mu \leq \mu_{0}$ and every $x_{0} \in \mathbb{R}$, we have

$$
\left\|\left(\mathbb{T}_{\mu, x_{0}} \circ \Pi-\Pi \circ \mathbb{T}_{\mu, x_{0}}\right) u\right\|_{W_{\phi\left(\varphi_{\mu, x_{0}}\right)^{-1}}^{l+1, p}(\Omega)} \leq C \mu\|u\|_{W_{\phi}^{l, p}(\Omega)}
$$

where the constant $C$ depends on $C_{\phi}$, but is independent of $\mu, u, x_{0}$ and on the concrete choice of the weight $\phi$. Moreover, the analogous result holds for the spaces $W_{b, \phi}^{l, p}(\Omega)$ as well. 
Proof. Indeed, let $\Phi$ and $\Phi_{\varphi}$ be the stream functions associated with divergent free vector fields $v:=\Pi u$ and $v_{\varphi}:=\Pi\left(\varphi_{\mu, x_{0}} u\right)$ respectively. Then, according to equation (4.9), the function $W:=\varphi_{\mu, x_{0}} \Phi-\Phi_{\varphi}$ solves

$$
\begin{aligned}
& \Delta_{x} W=h:=2 \varphi_{\mu, x_{0}}^{\prime} \partial_{x_{1}} \Phi+\varphi_{\mu, x_{0}}^{\prime \prime} \Phi+\varphi_{\mu, x_{0}}^{\prime} u_{2}, \\
& \left.W\right|_{\partial \Omega}=0 .
\end{aligned}
$$

Now using estimates (2.6) for the derivatives of $\varphi_{\mu, x_{0}}$ and estimate (4.10) for $\Phi$, we obtain

$$
\|h\|_{W_{\phi\left(\varphi_{\mu, x_{0}}\right)^{-1}}^{l, p}(\Omega)} \leq C \mu\|u\|_{W_{\phi}^{l, p}(\Omega)}
$$

where the constant $C$ is independent of $\mu, x_{0}$ and on the concrete choice of the weight $\phi$. Applying now Proposition 3.1 to equation (4.18), we arrive at

$$
\|W\|_{W_{\phi\left(\varphi, x_{0}\right)^{-1}}^{l+2, p}(\Omega)} \leq C_{1} \mu\|u\|_{W_{\phi}^{l, p}(\Omega)} .
$$

In order to verify estimate (4.17) it is now sufficient to recall that

$$
\varphi_{\mu, x_{0}} v_{1}-v_{\varphi, 1}=\partial_{x_{2}} W, \varphi_{\mu, x_{0}} v_{2}-v_{\varphi, 2}=-\partial_{x_{1}} W+\varphi_{\mu, x_{0}}^{\prime} \Phi
$$

Thus, (4.17) is proved. For the case of the spaces $W_{b, \phi}^{l, p}(\Omega)$, the proof of (4.17) is completely analogous. Proposition 4.7 is proved.

We now consider the elliptic operator $A:=\Pi \Delta_{x}$ defined on the space of divergent free vector fields and formulate the natural regularity result for this operator.

Proposition 4.8. Let $\Omega$ be a strip and let $A:=\Pi \Delta_{x}$. Then, for every $1<p<\infty$ and $l=0,1$ there exists positive $\mu_{0}=\mu_{0}(p)$ such that, for every weight function of $a$ sufficiently small exponential growth rate $\left(\mu \leq \mu_{0}\right)$, operator A realizes an isomorphism between spaces $\mathcal{V}_{\phi}^{p}(\Omega) \cap \mathcal{H}_{\phi}^{l+2, p}(\Omega)$ and $\mathcal{H}_{\phi}^{l, p}(\Omega)$ and the following estimate holds:

$$
C^{-1}\|u\|_{\mathcal{H}_{\phi}^{l+2, p}(\Omega)} \leq\left\|\Pi \Delta_{x} u\right\|_{\mathcal{H}_{\phi}^{l, p}(\Omega)} \leq C\|u\|_{\mathcal{H}_{\phi}^{l+2, p}(\Omega)}
$$

where the constant $C$ depends on $C_{\phi}$, but is independent of the concrete choice of the weight function $\phi$. Moreover, the analogous result holds for the spaces $\mathcal{H}_{b, \phi}^{l, p}(\Omega)$ as well.

Proof. We first note that the right-hand side of (4.21) is an immediate corollary of Theorem 4.4, so, we only need to verify the left one.

Indeed, let $g=\Pi \Delta_{x} u, u \in \mathcal{H}_{\phi}^{l+2, p}(\Omega)$. Due to the decomposition (4.7) and Theorem 4.4, that is equivalent to the following stationary Stokes equation in $\Omega$ :

$$
\begin{aligned}
& \Delta_{x} u+\nabla_{x} p=g, \\
& \left.u\right|_{\partial \Omega}=0, \quad \operatorname{div} u=0 .
\end{aligned}
$$

In order to solve (4.22), we use again the stream function $\Phi$ for the divergent free vector field $u$. Then, the function $\Phi$ should satisfy the following biLaplace equation in $\Omega$ :

$$
\Delta_{x}^{2} \Phi=\partial_{x_{2}} g_{1}-\partial_{x_{1}} g_{2},\left.\quad \Phi\right|_{\partial \Omega}=\left.\partial_{n} \Phi\right|_{\partial \Omega}=0
$$


Vice versa, let $\Phi$ solve (4.23) and let $u=\left(\partial_{x_{2}} \Phi,-\partial_{x_{1}} \Phi\right)$. Then, the vector field $w:=$ $\Delta_{x} u-g$ satisfies (4.11) and, consequently, it is potential and $u$ solves (4.22). Thus, problems (4.22) and (4.23) are equivalent.

It only remains to note that, due to Proposition 4.7, problem (4.23) is uniquely solvable and

$$
\|\Phi\|_{W_{\phi}^{l+3, p}(\Omega)} \leq C\left\|\partial_{x_{2}} g_{1}-\partial_{x_{1}} g_{2}\right\|_{W_{\phi}^{l-1, p}(\Omega)} \leq C_{1}\|g\|_{\mathcal{H}_{\phi}^{l, p}(\Omega)}
$$

which, together with (4.3) and the fact that $g=\Pi \Delta_{x} u$ gives the left-hand side of (4.21) and finishes the proof of Proposition 4.8.

COROLlary 4.9. Let the assumptions of Proposition 4.8 hold and let $p=2$. Then, for every weight function with a sufficiently small growth rate $\mu$, we have

$$
C^{-1}\left(\phi \Delta_{x} u, \phi \Delta_{x} u\right) \leq\left(\phi \Pi \Delta_{x} u, \phi \Pi \Delta_{x} u\right) \leq C\left(\phi \Delta_{x} u, \phi \Delta_{x} u\right)
$$

where $(\cdot, \cdot)$ denotes the standard inner product in $\left[L^{2}(\Omega)\right]^{2}$ and the constant $C$ is independent of the concrete choice of the weight $\phi$ and $u \in \mathcal{V}_{\phi}^{2}(\Omega) \cap \mathcal{H}_{\phi}^{2,2}(\Omega)$.

Indeed, estimate (4.25) is an immediate corollary of (4.21) with $p=2$ and the following elliptic regularity estimate for the Laplacian in $\Omega$ with Dirichlet boundary conditions:

$$
C^{-1}\|u\|_{W_{\phi}^{2,2}(\Omega)} \leq\left\|\Delta_{x} u\right\|_{L_{\phi}^{2}(\Omega)} \leq C\|u\|_{W_{\phi}^{2,2}(\Omega)},
$$

see Proposition 3.1.

We conclude this section by considering the action of operator $A:=\Pi \Delta_{x}$ in weaker spaces $\mathcal{V}_{\phi}^{p}$ which will be used in the sequel in order to define weak weighted energy solutions of the Navier-Stokes equations and derive the appropriate energy estimates. To this end, we need to define the corresponding functional spaces.

Definition 4.10 . Let $\Omega$ be a strip and let $\mathcal{D}_{\text {div }}(\Omega)$ be the space of all smooth divergent free vector fields in $\Omega$ with compact support. As usual, we denote by $\mathcal{D}_{\text {div }}^{\prime}(\Omega)$ the space of all linear continuous functionals on $\mathcal{D}_{\text {div }}(\Omega)$. We denote also by $\mathcal{H}^{-1, p}\left(\Omega_{s}\right) \subset \mathcal{D}_{\text {div }}^{\prime}\left(\Omega_{s}\right)$ the conjugate space to $\mathcal{V}^{q}\left(\Omega_{s}\right)$ with the standard norm.

Finally, for every weight function $\phi$ of exponential growth rate $\mu$, we define the spaces $\mathcal{H}_{\phi}^{-1}(\Omega)$ and $\mathcal{H}_{b, \phi}^{-1}(\Omega)$ as subspaces of $\mathcal{D}_{\text {div }}^{\prime}(\Omega)$ with the following finite norms:

$$
\begin{aligned}
\|u\|_{\mathcal{H}_{\phi}^{-1, p}(\Omega)}^{p} & :=\int_{s \in \Omega} \phi(s)^{p}\|u\|_{\mathcal{H}^{-1, p}\left(\Omega_{s}\right)}^{p} d s<\infty, \\
\|u\|_{\mathcal{H}_{b, \phi}^{-1, p}(\Omega)} & :=\sup _{s \in \mathbb{R}}\left\{\phi(s)\|u\|_{\mathcal{H}^{-1, p}\left(\Omega_{s}\right)}\right\}<\infty .
\end{aligned}
$$

Arguing exactly as in Proposition 2.8, one can show that

$$
\mathcal{H}_{\phi}^{-1, p}(\Omega)=\left[\mathcal{V}_{\phi^{-1}}^{q}(\Omega)\right]^{*}
$$

We note however that the spaces $\mathcal{H}_{\phi}^{-1, p}(\Omega)$ are not the subspaces of distributions and, in fact larger than the corresponding spaces $\left[W_{\phi}^{-1, p}(\Omega)\right]^{2}$ of distributions. Nevertheless, there is a natural map of $\left[W_{\phi}^{-1, p}(\Omega)\right]^{2}$ to $\mathcal{H}_{\phi}^{-1, p}(\Omega)$ (which is usually considered as an 
extension of the projector $\Pi$ to the negative Sobolev spaces and is also denoted by $\Pi$ )

$$
\langle\Pi u, v\rangle_{\operatorname{div}}:=\langle u, v\rangle, \operatorname{div} v=0
$$

where in the left-hand side we have the pairing in $\mathcal{D}_{\text {div }}^{\prime}(\Omega) \times \mathcal{D}_{\text {div }}(\Omega)$ and in the righthand side the standard pairing in distributional sense is written.

Thus, the Stokes operator $A=\Pi \Delta_{x}$ can be naturally extended to the operator from $\mathcal{V}_{\phi}^{p}(\Omega)$ to $\mathcal{H}_{\phi}^{-1, p}(\Omega)$ (and, analogously, in the spaces $\mathcal{V}_{b, \phi}^{p}(\Omega)$ ). The last result of that section shows that this operator is an isomorphism, i.e., Proposition 4.8 holds for $l=-1$ as well.

Proposition 4.11. Let $\Omega$ be a strip and let $A=\Pi \Delta_{x}$ be as above. Then, for every $1<p<\infty$, there exists positive $\mu_{0}=\mu_{0}(p)$ such that, for every weight function $\phi$ with a sufficiently small exponential growth rate $\mu\left(\mu \leq \mu_{0}\right)$, the operator $A$ realizes an isomorphism between spaces $\mathcal{V}_{\phi}^{p}(\Omega)$ and $\mathcal{H}_{\phi}^{-1, p}(\Omega)$ and the following estimate holds:

$$
C^{-1}\|u\|_{\mathcal{V}_{\phi}^{p}(\Omega)} \leq\left\|\Pi \Delta_{x} u\right\|_{\mathcal{H}_{\phi}^{-1, p}(\Omega)} \leq C\|u\|_{\mathcal{V}_{\phi}^{p}(\Omega)}
$$

where the constant $C$ depends on $C_{\phi}$, but is independent of $u$ and of the concrete choice of the weight $\phi$. Moreover, the analogous result holds for the spaces $\mathcal{V}_{b, \phi}^{p}(\Omega)$ as well.

Proof. As it was established in Proposition 4.2, maps (4.3) and (4.4) give an isomorphism between spaces $\mathcal{V}_{\phi^{-1}}^{q}(\Omega)$ and $W_{0, \phi^{-1}}^{2, q}(\Omega)$. This isomorphism naturally generates an isomorphism of the conjugated spaces, namely, between $W_{\phi}^{-2, p}(\Omega)$ and $\mathcal{H}_{\phi}^{-1, p}(\Omega)$. Indeed, let $g \in \mathcal{H}_{\phi}^{-1, p}(\Omega)$. Then, the associated functional $\bar{g} \in W_{\phi}^{-2, p}(\Omega)$ is defined via

$$
\langle\bar{g}, \Phi\rangle:=\left\langle g,\left(\partial_{x_{2}} \Phi,-\partial_{x_{1}} \Phi\right)\right\rangle_{\mathrm{div}}=-\left\langle\partial_{x_{2}} g_{1}-\partial_{x_{1}} g_{2}, \Phi\right\rangle
$$

Vice versa, for every $\bar{g} \in W_{\phi}^{-2, p}(\Omega)$, one defines the associated functional $g \in \mathcal{H}_{\phi}^{-1, p}(\Omega)$ by

$$
\langle g, v\rangle_{\operatorname{div}}:=\left\langle\bar{g}, \Phi_{v}\right\rangle
$$

where $\Phi_{v}$ is a stream function associated with $v$. Moreover, the operator $A=\Pi \Delta_{x}$ is conjugated to the biLaplacian under that isomorphism. Indeed, we have

$$
\left\langle\Delta_{x} u, v\right\rangle_{\mathrm{div}}=-\left\langle\Delta_{x}^{2} \Phi_{u}, \Phi_{v}\right\rangle
$$

and, consequently, equality $\Pi \Delta_{x} u=g$ reads

$$
\Delta_{x}^{2} \Phi_{u}=-\bar{g}=\partial_{x_{2}} g_{1}-\partial_{x_{1}} g_{2},\left.\quad \Phi\right|_{\partial \Omega}=\left.\partial_{n} \Phi\right|_{\partial \Omega}=0 .
$$

Thus, due to Proposition 3.3, we have

$$
\left\|\Phi_{u}\right\|_{W_{\phi}^{2, p}(\Omega)} \leq C\|\bar{g}\|_{W_{\phi}^{-2, p}(\Omega)} \leq C_{1}\|g\|_{\mathcal{H}_{\phi}^{-1, p}(\Omega)}
$$

and the analogous estimate for the spaces $W_{b, \phi}^{2, p}(\Omega)$. This estimate finishes the proof of the left-hand side of (4.29). Since the right-hand side of it is obvious, Proposition 4.11 is proved. 
5. An auxiliary linear Stokes problem. In this section, we study the following nonstationary linear Stokes problem in a strip $\Omega$ :

$$
\begin{aligned}
\partial_{t} w & =\Delta_{x} w-\nabla_{x} q, \\
\operatorname{div} w & =h(t), \quad S w_{1} \equiv 0 \\
\left.w\right|_{\partial \Omega} & =0,\left.\quad \Pi w\right|_{t=0}=0
\end{aligned}
$$

where $h(t)=h(t, x)$ is a given function satisfying

$$
\operatorname{Sh}(t)\left(x_{1}\right) \equiv 0, \quad t \in[0, T], \quad x_{1} \in \mathbb{R} .
$$

This auxiliary problem will be essentially used in the next section in order to obtain the weighted energy estimates for weak solutions of the nonlinear Navier-Stokes system. (5.1).

The following theorem gives a priori estimates and the solvability result for problem

THEOREM 5.1. There exists a positive $\mu_{0}$ such that, for every weight function $\phi$ of sufficiently small exponential growth rate $\mu\left(\mu \leq \mu_{0}\right)$ and every

$$
h \in L^{2}\left([0, T], W_{\phi}^{1,2}(\Omega)\right) \cap C\left([0, T], L_{\phi}^{2}(\Omega)\right)
$$

for which (5.2) is satisfied, problem (5.1) possesses a unique solution $w$ from the class

$$
\begin{aligned}
& w \in L^{2}\left([0, T], W_{\phi}^{2,2}(\Omega)\right) \cap C\left([0, T], W_{\phi}^{1,2}(\Omega)\right), \\
& \partial_{t} \Pi w \in L^{2}\left([0, T], L_{\phi}^{2}(\Omega)\right), \quad q \in \mathcal{D}^{\prime}([0, T] \times \Omega)
\end{aligned}
$$

and satisfying the following estimates:

$$
\begin{gathered}
\int_{0}^{T} e^{-\alpha|t-s|}\left(\left\|\partial_{t} \Pi w(s)\right\|_{L_{\phi}^{2}(\Omega)}^{2}+\|w(s)\|_{W_{\phi}^{2,2}(\Omega)}^{2}\right) d s \leq C \int_{0}^{T} e^{-\alpha|t-s|}\|h(s)\|_{W_{\phi}^{1,2}(\Omega)}^{2} d s, \\
\|w(t)\|_{W_{\phi}^{1,2}(\Omega)}^{2} \leq C\left(\|h(t)\|_{L_{\phi}^{2}(\Omega)}^{2}+\int_{0}^{T} e^{-\alpha|t-s|}\|h(s)\|_{W_{\phi}^{1,2}(\Omega)}^{2} d s\right)
\end{gathered}
$$

where $\alpha$ is a sufficiently small positive constant depending only on $\mu_{0}$ and the constant $C$ depends on $C_{\phi}$, but is independent of the concrete choice of the weight $\phi$.

Proof. In order to solve (5.1), we are going to reduce it to the divergent free case. To this end, we need the following lemma.

LEMMA 5.2. Let the above assumptions hold and let us consider the following stationary Stokes problem:

$$
\Delta_{x} v-\nabla_{x} r=0, \quad \operatorname{div} v=h,\left.\quad v\right|_{\partial \Omega}=0 .
$$

Then there exists positive $\mu_{0}$ such that, for every weight function of sufficiently small exponential growth rate $\mu\left(\mu \leq \mu_{0}\right)$ and every $h \in W_{\phi}^{l, 2}(\Omega), l=0,1$ satisfying (5.2), equation (5.6) possesses a unique solution $v \in W_{\phi}^{l+1,2}(\Omega)$, $\mathbb{S} v_{1} \equiv 0$, satisfying (5.6) in the sense of distributions and the following estimate holds:

$$
\|v\|_{W_{\phi}^{l+1,2}(\Omega)} \leq C\|h\|_{W_{\phi}^{l, 2}(\Omega)}
$$

where the constant $C$ depends on $C_{\phi}$, but is independent of the concrete choice of the weight $\phi$. 
Proof. Let us define the function $K$ as a unique solution of the following problem:

$$
\Delta_{x} K=h,\left.\quad \partial_{n} K\right|_{\partial \Omega}=0, \quad \mathbb{S} K \equiv 0
$$

(which exists due to condition (5.2) and Proposition (3.5) and let $\theta:=\nabla_{x} K$. Then, obviously,

$$
\operatorname{div} \theta=h, \quad l_{n} \theta=\left.\theta_{2}\right|_{\partial \Omega}=0
$$

and, due to Propositions 2.13 and 3.5 , we have

$$
\|\theta\|_{W_{\phi}^{l+1,2}(\Omega)}+\left\|\left.\theta\right|_{\partial \Omega}\right\|_{W_{\phi}^{l+1 / 2,2}(\partial \Omega)} \leq C\|h\|_{W_{\phi}^{l, 2}(\Omega)} .
$$

Let us now put $\bar{v}:=v-\theta$. Then, this function should satisfy

$$
\Delta_{x} \bar{v}-\nabla_{x} \bar{r}=0, \operatorname{div} \bar{v}=0,\left.l_{n} \bar{v}\right|_{\partial \Omega}=0,\left.\bar{v}_{1}\right|_{\partial \Omega}=-\left.\theta_{1}\right|_{\partial \Omega}, \quad S v_{1} \equiv 0 .
$$

In particular, we see that $\bar{v} \in \mathcal{H}_{\phi}^{l, 2}(\Omega)$ and, consequently, we can use transformation (4.3) and (4.4) to the associated stream function $\Phi$ which should satisfy the following equation:

$$
\Delta_{x}^{2} \Phi=0,\left.\quad \Phi\right|_{\partial \Omega}=0,\left.\quad \partial_{n} \Phi\right|_{\partial \Omega}=-\left.\theta_{1}\right|_{\partial \Omega} .
$$

The assertion of the lemma is now an immediate corollary of estimate (5.10) and Proposition 3.3.

We are now ready to finish the proof of the theorem. To this end, we introduce a new dependent variable $\bar{w}(t):=w(t)-v(t)$ where, for every $t \in[0, T]$ function $v(t)$ solves the stationary problem (5.6) with $h$ replaced by $h(t)$. This function obviously satisfies the following equation:

$$
\partial_{t}(\bar{w}+v)=\Delta_{x} \bar{w}-\nabla_{x} \bar{q}, \quad \operatorname{div} \bar{w}=0,\left.\bar{w}\right|_{\partial \Omega}=0,\left.\bar{w}\right|_{t=0}=-\left.\Pi v\right|_{t=0} .
$$

Applying the projector $\Pi$ to both parts of (5.13), we obtain

$$
\partial_{t}(\bar{w}+\Pi v)=\Pi \Delta_{x} \bar{w}, \quad \operatorname{div} \bar{w}=0,\left.\quad \bar{w}\right|_{\partial \Omega}=0,\left.\quad \bar{w}\right|_{t=0}=-\left.\Pi v\right|_{t=0} .
$$

In order to obtain an a priori estimate for solutions of (5.14), we multiply it by the expression $\varphi_{2 \mu, x_{0}}\left(x_{1}\right) \partial_{x_{2}}^{2}(\bar{w}+\Pi v)+\partial_{x_{1}}\left[\varphi_{2 \mu, x_{0}}\left(x_{1}\right) \partial_{x_{1}}(\bar{w}+\Pi v)\right]$ where $x_{0} \in \mathbb{R}$ is arbitrary, $\mu>0$ is small enough and the weight $\varphi$ is defined by (2.4). Then, we get

$$
\begin{aligned}
& 1 / 2 \partial_{t}\left(\varphi_{2 \mu, x_{0}},\left|\nabla_{x}(\bar{w}+\Pi v)\right|^{2}\right)+\left(\varphi_{2 \mu, x_{0}} \Pi \Delta_{x} \bar{w}, \Delta_{x} \bar{w}\right) \\
& \quad=-\left(\varphi_{2 \mu, x_{0}}^{\prime} \Pi \Delta_{x} \bar{w}, \partial_{x_{1}} \bar{w}\right)-\left(\varphi_{2 \mu, x_{0}} \Pi \Delta_{x} \bar{w}, \Delta_{x} \Pi v\right)-\left(\varphi_{2 \mu, x_{0}}^{\prime} \Pi \Delta_{x} \bar{w}, \partial_{x_{1}} \Pi v\right) .
\end{aligned}
$$

We estimate the second term in the left-hand side of (5.15) using estimates (4.17), (4.8) and (4.25) in the following way:

$$
\begin{aligned}
\left(\varphi_{2 \mu, x_{0}} \Pi \Delta_{x} \bar{w}, \Delta_{x} \bar{w}\right) & =\left(\varphi_{2 \mu, x_{0}},\left|\Pi \Delta_{x} \bar{w}\right|^{2}\right)-\left(\Pi \Delta_{x} \bar{w},\left(\varphi_{2 \mu, x_{0}} \circ \Pi-\Pi \circ \varphi_{2 \mu, x_{0}}\right) \Delta_{x} \bar{w}\right) \\
& \geq C\left(\varphi_{2 \mu, x_{0}},\left|\Delta_{x} \bar{w}\right|^{2}\right)-C_{1}\left(\varphi_{-2 \mu, x_{0}},\left|\left(\varphi_{2 \mu, x_{0}} \circ \Pi-\Pi \circ \varphi_{2 \mu, x_{0}}\right) \Delta_{x} \bar{w}\right|^{2}\right) \\
& \geq\left(C_{2}-C_{3} \mu\right)\left\|\Delta_{x} \bar{w}\right\|_{L_{\varphi_{\mu, x}}^{2}(\Omega)}^{(5.16)}
\end{aligned}
$$


where the constants $C_{i}$ are independent of $\mu$ and $x_{0}$. Fixing $\mu$ now to be small enough, estimating the right-hand side of (5.15) by Hölder inequality and using (4.8) and (4.21), we have

$$
\begin{aligned}
\partial_{t}\left(\left\|\nabla_{x}(\bar{w}+\Pi v)\right\|_{L_{\varphi_{\mu, x_{0}}}^{2}(\Omega)}^{2}\right) & +\alpha\left(\left\|\Delta_{x} \bar{w}\right\|_{L_{\varphi_{\mu}, x_{0}}^{2}(\Omega)}^{2}\right. \\
& \left.+\left\|\nabla_{x}(\bar{w}+\Pi v)\right\|_{L_{\varphi_{\mu, x_{0}}}^{2}(\Omega)}^{2}\right) \leq C\|v\|_{W_{\varphi_{\mu}, x_{0}}^{2,2}(\Omega)}^{2},
\end{aligned}
$$

where the positive constants $\alpha$ and $C$ are independent of $x_{0} \in \mathbb{R}$ (here we have also implicitly used $\left.\left\|\nabla_{x}(\bar{w}+\Pi v)\right\|_{L_{\varphi_{\mu}, x_{0}}^{2}(\Omega)} \leq C\left(\left\|\nabla_{x} \bar{w}\right\|_{L_{\varphi_{\mu, x_{0}}}^{2}(\Omega)}+\|v\|_{W_{\varphi_{\mu, x_{0}}^{2}(\Omega)}^{2,2}}\right)\right)$.

Applying the Gronwall inequality to (5.17) and using estimate (5.7) with $l=1$ (for every fixed $t$ ), we arrive at

$$
\begin{aligned}
\left\|\nabla_{x}(\bar{w}(t)+\Pi v(t))\right\|_{L_{\varphi_{\mu, x_{0}}}^{2}(\Omega)}^{2} & +\int_{0}^{t} e^{-\alpha(t-s)}\|\bar{w}(s)\|_{W_{\varphi_{\mu, x_{0}}}^{2,2}(\Omega)}^{2} d s \\
& \leq C \int_{0}^{t} e^{-\alpha(t-s)}\|h(s)\|_{W_{\varphi_{\mu, x_{0}}}^{1,2}(\Omega)}^{2} d s
\end{aligned}
$$

(here we have also used $\bar{w}(0)+\Pi v(0)=\Pi u(0)=0)$. Moreover, since the constant $C$ in (5.18) is independent of $x_{0} \in \mathbb{R}$, then, multiplying (5.18) by $\phi^{2}\left(x_{0}\right)$, integrating over $x_{0} \in \mathbb{R}$ and using (2.12), we obtain (exactly as in Section 3 ) the analogue of estimate (5.18) not only for the special weights $\varphi_{\mu, x_{0}}$, but also for arbitrary weight $\phi$ of exponential growth rate $\varepsilon<\mu$.

In order to deduce a priori estimate (5.5) from (5.18), it only remains to recall that $w=\bar{w}+v$ and (due to (5.7) with $l=0$ )

$$
\|w(t)\|_{W_{\phi}^{1,2}(\Omega)} \leq C\left(\left\|\nabla_{x}(\bar{w}(t)+\Pi v(t))\right\|_{L_{\phi}^{2}(\Omega)}+\|h(t)\|_{L_{\phi}^{2}(\Omega)}\right) .
$$

Indeed, this estimate together with (5.18) gives the required estimate for the $W_{\phi}^{1,2}$-norm of $w(t)$; the estimate for the $W_{\phi}^{2,2}$-norm of $w$ is also an immediate corollary of (5.18) and (5.7) with $l=1$. Finally, the required estimate for $\partial_{t} \Pi w=\partial_{t}(\bar{w}+\Pi v)$ can be now obtained from equation (5.14). Thus, a priori estimate (5.5) is proved.

We also note that, due to our construction, we have

$$
v(t)=\Pi v(t)+\nabla_{x} K(t)
$$

where $K(t, x)=K_{h(t)}(x)$ solves problem (5.8) for every fixed $t$. Thus,

$$
(\mathrm{Id}-\Pi) \partial_{t} w(t)=\partial_{t} \nabla_{x} K_{h}(t)=\nabla_{x} K_{\partial_{t}} h(t),
$$

and we see that, in contrast to the divergence free component of $\partial_{t} u$ its potential component does not belong to $L_{\phi}^{2}(\Omega)$ for general external forces $h$, but if, in addition, we have $\partial_{t} h \in L_{\phi}^{2}(\Omega)$, then (5.20) and Lemma 5.2 show that $\partial_{t} u$ will be also in $L_{\phi}^{2}(\Omega)$ and equation (5.1) can be naturally understood as an equality in $L^{2}\left([0, T], L_{\phi}^{2}(\Omega)\right)$.

The above observation gives a natural way to construct the required solution $w(t)$ of (5.1) based on the obtained a priori estimate. Indeed, let us approximate the external force $h \in C\left([0, T], L_{\phi}^{2}(\Omega)\right) \cap L^{2}\left([0, T], W_{\phi}^{1,2}(\Omega)\right)$ by a sequence of smooth (with respect to $t$ and $x$ ) functions $h^{n}$ having the compact support in $x_{1}$ and satisfying (5.2). Having such $h^{n}$, we construct the associated functions $v^{n} \in C^{1}\left([0, T], W^{2,2}(\Omega)\right)$ by Lemma 5.2. 
Then, the associated equation (5.13) for $\bar{w}^{n}$ will be the standard nonstationary Stokes equation with the external forces $\partial_{t} v(t)$ belonging to the unweighted space $C\left([0, T], W^{2,2}(\Omega)\right)$.

It is well-known that, for such external forces the nonstationary Stokes equation possesses a unique solution $\bar{w}^{n} \in W^{1,2}\left([0, T], L^{2}(\Omega)\right) \cap L^{2}\left([0, T], W^{2,2}(\Omega)\right)$, see e.g. [4] or [5]. Thus, the approximating sequence of solutions $w^{n}$ is constructed. We also note that, since $w^{n}(t)$ belongs to $L^{2}(\Omega)$ and is divergent free, one has

$$
\mathfrak{S} \bar{w}_{1}^{n} \equiv 0 \text { and, consequently } \mathbb{S} w_{1}^{n} \equiv 0 .
$$

Moreover, since the $h^{n}$ have compact support in $x_{1}$, then a priori estimate (5.5) holds for $w^{n}$ uniformly with respect to $n \rightarrow \infty$. Passing now to the limit $n \rightarrow \infty$ and using (5.21) we construct the required solution $w(t)$. Theorem 5.1 is proved.

REMARK 5.3. Condition $\mathbb{S} w_{1} \equiv 0$ is essential for the uniqueness part of Theorem 5.1. As we will see below, for every function $c(t) \in C_{b}(R)$, equation (5.1) possesses a solution $w$ satisfying $\mathbb{S} w_{1}(t) \equiv c(t)$.

The next corollary gives one more estimate for the solutions of problem (5.1) which will be used in Section 7 in order to verify the uniqueness theorem for the nonlinear Navier-Stokes problem.

COROLlary 5.4. Let the assumptions of Theorem 5.1 hold. Then, the solution $w(t)$ of problem (5.1) satisfies the following estimate:

$$
\int_{0}^{t}\|w(\tau)\|_{L_{\phi}^{2}(\Omega)}^{2} d \tau \leq C \int_{0}^{t}\|h(\tau)\|_{L_{\phi}^{2}(\Omega)}^{2} d \tau
$$

where $\phi$ is a weight function of sufficiently small exponential growth rate and the constant $C$ depends on $C_{\phi}$, but is independent of the concrete choice of $h$ and $\phi$.

Proof. Indeed, due to Lemma 5.2, it is sufficient to verify estimate (5.22) only for the solution $\bar{w}(t)$ of equation (5.14). In order to do so, we multiply this equation by $e^{-(s-t)}$, integrate over $t \in[0, s]$ and introduce a new dependent variable $Z(s):=$ $\int_{0}^{s} e^{-(s-t)} \bar{w}(t) d t$. Then, this function satisfies the following equation:

$$
\partial_{s} Z(s)-\Pi \Delta_{x} Z(s)=H(s), \operatorname{div} Z(s)=0, \quad Z(0)=0
$$

where $H(s):=\int_{0}^{s} e^{-(s-t)} \Pi v(t) d t-\Pi v(s)$.

Arguing now as in the end of the proof of Theorem 5.1 (see (5.15)-(5.18)), we deduce that the solution $Z(s)$ of equation (5.23) satisfies

$$
\int_{0}^{s}\left\|\partial_{s} Z(\tau)\right\|_{L_{\phi}^{2}(\Omega)}^{2}+\|Z(\tau)\|_{W_{\phi}^{2,2}(\Omega)}^{2} d \tau \leq C \int_{0}^{s}\|H(\tau)\|_{L_{\phi}^{2}(\Omega)}^{2} d \tau .
$$

Moreover, due to Lemma 5.2, we have also the estimate

$$
\int_{0}^{s}\|H(\tau)\|_{L_{\phi}^{2}(\Omega)}^{2} d \tau \leq C \int_{0}^{s}\|\Pi v(\tau)\|_{L_{\phi}^{2}(\Omega)}^{2} d \tau \leq C_{1} \int_{0}^{s}\|h(\tau)\|_{L_{\phi}^{2}(\Omega)}^{2} d \tau .
$$

Combining estimates (5.24) and (5.25) and taking into account the evident relation $\bar{w}(s)=\partial_{s} Z(s)+Z(s)$, we derive estimate (5.22) for the function $\bar{w}(t)$. Corollary 5.4 is proved. 
We conclude this section by preparing some technical tools for obtaining the energy estimates for the nonlinear Navier-Stokes equation in a strip. To this end, we need to introduce some more functional spaces.

DEFINITION 5.5. Let $\Omega$ be a strip and let $\mathbb{W}_{b}([0, T] \times \Omega)$ consists of vector fields $u \in L_{b}^{2}\left([0, T], \mathcal{V}_{b}^{2}(\Omega)\right) \cap L_{b}^{4}\left([0, T], \mathcal{H}_{b}^{4}(\Omega)\right.$ ) (see Remark 2.4) such that the $t$-derivative $\partial_{t} u$ belongs to $\mathcal{D}_{\text {div }}^{\prime}(\Omega)$ a.e. and satisfies

$$
\partial_{t} u \in L_{b}^{2}\left([0, T], \mathcal{H}_{b}^{-1,2}(\Omega)\right)+L_{b}^{4 / 3}\left([0, T], \mathcal{H}_{b}^{4 / 3}(\Omega)\right) .
$$

We recall that, as usual, the space $U+V$ is defined as the space of functions (functionals) $\phi$ which can be presented in the form $\phi=u+v$ where $u \in U, v \in V$ with the norm

$$
\|\phi\|_{U+V}:=\inf \left\{\|u\|_{U}+\|v\|_{V} ; u \in U, v \in V, u+v=\phi\right\} .
$$

Let us consider also an arbitrary weight function $\theta$ of a sufficiently small exponential growth rate $\mu$ and a smooth nonnegative function $\phi$ satisfying the following assumptions:

$$
\left|\phi^{\prime}(s)\right|+\phi(s) \leq C \theta(s), \quad s \in \mathbb{R}, \quad \int_{s \in \mathbb{R}} \theta^{2}(s) d s<\infty .
$$

In order to obtain the weighted energy estimates for the solution $u \in \mathbb{W}_{b}([0, T] \times \Omega)$ of the Navier-Stokes equation in $L_{\phi}^{2}(\Omega)$ (which contains $L_{b}^{2}(\Omega)$ due to the integrability assumption on $\phi$ ), it would be natural to multiply it by the function $\phi^{2} u$ and integrate over $\Omega$, but, unfortunately, this function is no more divergent free and, consequently, this way does not allow to exclude the pressure. Instead of that, we will multiply it by the function $\phi^{2} u-v$ where $v(t):=\left(\mathbb{P}_{\phi} u\right)(t)$ is the appropriate corrector which makes this multiplier divergent free. To this end, the function $v(t)$ should satisfy

$$
\operatorname{div} v(t) \equiv h_{u}(t):=2 \phi \phi^{\prime} u_{1}(t)
$$

(here we have used that $\operatorname{div} u=0$ ). Due to the integrability assumption on $\phi$, the function $h \in L^{2}\left([0, T], W_{\theta^{-1}}^{1,2}(\Omega)\right)$ and, moreover, since $\mathbb{S} u_{1} \equiv 0$, we have $\mathbb{S} h \equiv 0$ and (5.2) is satisfied.

Furthermore, it is convenient for us to fix the corrector $v(t):=\left(\mathbb{P}_{\phi} u\right)(t)$ as a solution of the following auxiliary nonstationary Stokes problem in $\Omega$ :

$$
-\partial_{t} v=\Delta_{x} v-\nabla_{x} q, \quad \operatorname{div} v(t)=h_{u}(t),\left.\quad v\right|_{\partial \Omega}=0,\left.\quad \Pi v\right|_{t=T}=0 .
$$

This equation, obviously, can be reduced to (5.1) by the time change $t \rightarrow T-t$. Thus, Theorem 5.1 and estimate (5.5) hold for this equation as well. The following theorem justifies our choice of the corrector $\mathbb{P}_{\phi}$ and gives the main technical tool for the weighted energy estimates of the Navier-Stokes equations.

THEOREM 5.6. Let $\Omega$ be a strip and let $\phi$ be a smooth nonnegative function, satisfying (5.28) for some square integrable weight $\theta$ of sufficiently small exponential growth rate $\mu$. Then

$$
\mathbb{W}_{b}([0, T] \times \Omega) \subset C\left([0, T], L_{\theta}^{2}(\Omega)\right)
$$


Let $\mathbb{P}_{\phi}$ be defined as the solving operator for problem (5.30). Then the following equality holds:

$$
\begin{aligned}
& \frac{d}{d t}\left[1 / 2\left(\phi^{2} u(t), u(t)\right)-\left(u(t),\left(\mathbb{P}_{\phi} u\right)(t)\right)\right]+\left(\nabla_{x} u(t), \nabla_{x}\left(\phi^{2} u(t)\right)\right) \\
& \quad=\left(\partial_{t} u(t)-\Pi \Delta_{x} u(t), \phi^{2} u-\left(\mathbb{P}_{\phi} u\right)(t)\right)
\end{aligned}
$$

which means that the function $1 / 2\left(\phi^{2} u, u\right)-\left(u, \mathbb{P}_{\phi} u\right)$ is absolutely continuous as a scalar function on $[0, T]$ and (5.32) holds almost everywhere.

Proof. Let $u \in \mathbb{W}_{b}([0, T] \times \Omega)$ be arbitrary and first let $\phi=\varphi_{\mu, x_{0}}$. Let us approximate $u$ by a uniformly bounded sequence $u_{n} \in \mathbb{W}_{b}([0, T] \times \Omega)$ of smooth with respect to $t$ functions such that

$$
\begin{gathered}
u_{n} \rightarrow u \text { in } L^{2}\left([0, T], \mathcal{V}_{\phi}^{2}(\Omega)\right) \cap L_{\phi}^{4}([0, T] \times \Omega) \text { and } \\
\partial_{t} u_{n} \rightarrow \partial_{t} u \text { in } L^{2}\left([0, T], \mathcal{H}_{\phi}^{-1,2}(\Omega)\right)+L_{\phi}^{4 / 3}([0, T] \times \Omega) .
\end{gathered}
$$

We recall that the initial function $u$ belongs to the spaces involved in (5.33) since $\phi=\varphi_{\mu, x_{0}}$ is exponentially decaying and, consequently, arguing in a standard way, it is not difficult to show that such approximation exists. Indeed, in order to do so, it is sufficient to extend the initial function $u \in \mathbb{W}_{b}([0, T] \times \Omega)$ till the function $\tilde{u} \in \mathbb{W}_{b}(\mathbb{R} \times \Omega$ ) (using e.g., the even extension through $t=0$ and $t=T$ ). The required approximating sequence $u_{n}$ can be constructed after that using the standard convolution operator with respect to $t$ :

$$
u_{n}(t):=\varepsilon_{n}^{-1} \int_{\tau \in \mathbb{R}} \Phi\left(\tau \varepsilon_{n}^{-1}\right) \tilde{u}(t-\tau) d \tau
$$

where $\varepsilon_{n} \rightarrow 0$ as $n \rightarrow \infty, \int_{\tau \in \mathbb{R}} \Phi(\tau) d \tau=1$ and supp $\Phi \subset[-1,1]$.

For smooth with respect to $t$ functions $u_{n}$ we can freely integrate by parts with respect to $t$ and verify (5.33). Indeed, since $\partial_{t} \Pi v_{n}+\Pi \Delta_{x} v_{n} \equiv 0$ and $\operatorname{div} u_{n}=\operatorname{div}\left(\phi^{2} u_{n}-\right.$ $\left.v_{n}\right)=0$, we have

$$
\begin{aligned}
& \left(\partial_{t} u_{n}-\Pi \Delta_{x} u_{n}, \phi^{2} u_{n}-v_{n}\right)=\left(\partial_{t} u_{n}-\Delta_{x} u_{n}, \phi^{2} u_{n}-v_{n}\right) \\
& \quad=\partial_{t}\left[1 / 2\left(\phi^{2} u_{n}, u_{n}\right)-\left(u_{n}, v_{n}\right)\right]+\left(\nabla_{x} u_{n}, \nabla_{x}\left(\phi^{2} u_{n}\right)\right)+\left(u_{n}, \partial_{t} v_{n}+\Delta_{x} v_{n}\right) \\
& \quad=\partial_{t}\left[1 / 2\left(\phi^{2} u_{n}, u_{n}\right)-\left(u_{n}, v_{n}\right)\right]+\left(\nabla_{x} u_{n}, \nabla_{x}\left(\phi^{2} u_{n}\right)\right) .
\end{aligned}
$$

Let us now prove embedding (5.31). Indeed, equality (5.34) has the form of $\frac{d}{d t} R_{n}(t)=$ $Q_{n}(t)$. Consequently, using the standard estimate

$$
\left|R_{n}(t)\right| \leq C \int_{0}^{T}\left(\left|Q_{n}(s)\right|+\left|R_{n}(s)\right|\right) d s, \quad T \geq 1
$$

for that equation, we deduce

$$
\begin{aligned}
\left|R_{n}(t)\right| \leq & C \int_{0}^{T}\left[\left(\nabla_{x}\left(\phi^{2} u_{n}(s)\right), \nabla_{x} u_{n}(s)\right.\right. \\
& \left.+\left\|v_{n}\right\|_{L_{\phi^{-1}}^{2}(\Omega)}^{2}+\left|\left(\partial_{t} u_{n}(s)-\Delta_{x} u_{n}(s), \phi^{2} u_{n}(s)-v_{n}(s)\right)\right|\right] d s
\end{aligned}
$$


where $R_{n}(s):=1 / 2\left(\phi^{2} u_{n}(s), u_{n}(s)\right)-\left(u_{n}(s), v_{n}(s)\right)$. We now recall that, due to Theorem 5.1 and (2.6), we have the following estimate:

$$
\begin{aligned}
& \left\|v_{n}(t)\right\|_{W_{\phi^{-1}}^{1,2}(\Omega)}^{2}+\int_{0}^{T} e^{-\alpha|t-s|}\left\|v_{n}(s)\right\|_{W_{\phi^{-1}}^{2,2}(\Omega)}^{2} d s \\
& \quad \leq C \mu\left(\left\|u_{n}(t)\right\|_{L_{\phi}^{2}(\Omega)}^{2}+\int_{0}^{T} e^{-\alpha|t-s|}\left\|u_{n}(s)\right\|_{W_{\phi}^{1,2}(\Omega)}^{2} d s\right) .
\end{aligned}
$$

This estimate shows that

$$
\begin{aligned}
R_{n}(s) & \geq 1 / 4\left\|u_{n}(s)\right\|_{L_{\phi}^{2}(\Omega)}^{2}-\left\|v_{n}(s)\right\|_{L_{\phi^{-1}}^{2}(\Omega)}^{2} \\
& \geq(1 / 4-C \mu)\left\|u_{n}(s)\right\|_{L_{\phi}^{2}(\Omega)}^{2}-C \mu \int_{0}^{T}\left\|u_{n}(s)\right\|_{W_{\phi}^{1,2}(\Omega)}^{2} d s .
\end{aligned}
$$

Fixing the constant $\mu$ to be small enough, inserting (5.37) into (5.35) and using (5.36) again, we get

$$
\left\|u_{n}(t)\right\|_{L_{\phi}^{2}(\Omega)}^{2} \leq C_{1} \int_{0}^{T}\left\|u_{n}(s)\right\|_{W_{\phi}^{1,2}(\Omega)}^{2} d s+\int_{0}^{T}\left|\left(\partial_{t} u_{n}(s), \phi^{2} u_{n}(s)-v_{n}(s)\right)\right| d s .
$$

Using now the fact that

$$
\begin{aligned}
& {\left[L^{2}\left([0, T], \mathcal{V}_{\phi^{-1}}^{2}(\Omega)\right) \cap L^{4}\left([0, T], \mathcal{H}_{\phi^{-1}}^{4}(\Omega)\right)\right]^{*}} \\
& \quad=L^{2}\left([0, T], \mathcal{H}_{\phi}^{-1,2}(\Omega)\right)+L^{4 / 3}\left([0, T], \mathcal{H}_{\phi}^{4 / 3}(\Omega)\right)
\end{aligned}
$$

(due to (2.17), (4.27), and the general topological fact that $[U \cap V]^{*}=U^{*}+V^{*}$, see [20]), we can estimate the last term in the right-hand side of (5.38) as follows:

$$
\begin{aligned}
& \int_{0}^{T}\left|\left(\partial_{t} u_{n}(s), \phi^{2} u_{n}(s)-v_{n}(s)\right)\right| d s \leq C\left\|\partial_{t} u_{n}\right\|_{L^{2}\left([0, T], \mathcal{H}_{\phi}^{-1,2}(\Omega)\right)+L^{4 / 3}\left([0, T], \mathcal{H}^{4 / 3}(\Omega)\right)} \\
& \quad \times\left\|\phi^{2} u_{n}-v_{n}\right\|_{L^{2}\left([0, T], \mathcal{V}_{\phi^{-1}}^{2}(\Omega)\right) \cap L_{\phi^{-1}}^{4}([0, T] \times \Omega)} \leq C\left\|u_{n}\right\|_{\mathbb{W}_{\phi}([0, T] \times \Omega)} \\
& \quad \times\left(\left\|u_{n}\right\| \mathbb{W}_{\phi}([0, T] \times \Omega)+\left\|v_{n}\right\|_{L^{2}\left([0, T], W_{\phi^{-1}}^{1,2}(\Omega)\right)}+\left\|v_{n}\right\|_{L_{\phi^{-1}}^{4}([0, T] \times \Omega)}\right) .
\end{aligned}
$$

The $W_{\phi^{-1}}^{1,2}$-norm of $v_{n}$ can be easily estimated by (5.36), so we only need to estimate its $L_{\phi^{-1}}^{4}$-norm. To this end, we will use the interpolation inequality (2.39) and again estimate (5.36). Then, we get

$$
\begin{aligned}
\left\|v_{n}\right\|_{L_{\phi^{-1}}^{4}([0, T] \times \Omega)}^{4} & \leq C\left\|v_{n}\right\|_{L^{\infty}\left([0, T], L_{\phi^{-1}}^{2}(\Omega)\right)}^{2}\left\|v_{n}\right\|_{L^{2}\left([0, T], W_{\phi^{-1}}^{1,2}(\Omega)\right)}^{2} \\
& \leq C_{1} \mu\left(\left\|u_{n}\right\|_{L^{\infty}\left([0, T], L_{\phi}^{2}(\Omega)\right)}^{4}+\left\|u_{n}\right\|_{L^{2}\left([0, T], W_{\phi}^{1,2}(\Omega)\right)}^{4}\right) .
\end{aligned}
$$

Inserting estimates (5.39) and (5.40) into the right-hand side of (5.38), we arrive at

$$
\left\|u_{n}\right\|_{L^{\infty}\left([0, T], L_{\phi}^{2}(\Omega)\right)}^{2} \leq C\left\|u_{n}\right\|_{\mathbb{W}_{\phi}([0, T] \times \Omega)}\left(\left\|u_{n}\right\|_{\mathbb{W}_{\phi}([0, T] \times \Omega)}+\left\|u_{n}\right\|_{L^{\infty}\left([0, T], L_{\phi}^{2}(\Omega)\right)}\right)
$$


and, consequently,

$$
\left\|u_{n}\right\|_{C\left([0, T], L_{\phi}^{2}(\Omega)\right)} \leq C\left\|u_{n}\right\|_{\mathbb{W}_{\phi}([0, T] \times \Omega)} .
$$

Applying now the same scheme for the function $u_{n}-u_{m}$ instead of $u_{n}$, we will have

$$
\left\|u_{n}-u_{m}\right\|_{C\left([0, T], L_{\phi}^{2}(\Omega)\right)} \leq C\left\|u_{n}-u_{m}\right\|_{\mathbb{W}_{\phi}([0, T] \times \Omega)} .
$$

Since $u_{n} \rightarrow u$ in $\mathbb{W}_{\phi}([0, T] \times \Omega)$ then the right-hand side of inequality (5.42) tends to zero as $m, n \rightarrow \infty$ and, consequently, $u_{n}$ is a Cauchy sequence in $C\left([0, T], L_{\phi}^{2}(\Omega)\right)$. Thus, we have proved that the limit function

$$
u \in C\left([0, T], L_{\varphi_{\mu, x_{0}}}^{2}(\Omega)\right)
$$

and

$$
\|u\|_{L^{\infty}\left([0, T], L_{\varphi_{\mu, x_{0}}}^{2}(\Omega)\right)} \leq C\|u\|_{\mathbb{W}_{\varphi_{\mu, x_{0}}}([0, T] \times \Omega)}
$$

(we recall that we have first considered the case of special weights $\varphi_{\mu, x_{0}}$ defined by (2.4)) where $\mu$ is small enough, $x_{0} \in \mathbb{R}$ and the constant $C$ is independent of $x_{0}$.

Taking now the supremum over $x_{0} \in \mathbb{R}$ from the both parts of (5.44) and using (2.13), we get

$$
\|u\|_{L^{\infty}\left([0, T], L_{b}^{2}(\Omega)\right)} \leq C\|u\|_{\mathbb{W}_{b}([0, T] \times \Omega)}
$$

and, consequently, $\mathbb{W}_{b}([0, T] \times \Omega) \subset L^{\infty}\left([0, T], L_{b}^{2}(\Omega)\right)$.

We now consider the case of general weights satisfying (5.28). We first note that the continuity (5.43) for the special weights together with estimate (5.45) and the fact that $\int_{s} \theta^{2}(s) d s<\infty$ imply in a standard way the continuity of $u(t)$ in the space $L_{\theta}^{2}(\Omega)$ : see [10] and Proposition 2.16. Thus, (5.31) is verified for general weights as well.

Let us now verify equality (5.32). To this end, it is sufficient to pass to the limit $n \rightarrow \infty$ in the integrated version of (5.34)

$$
\begin{aligned}
R_{n}(t)-R_{n}(0)=\int_{0}^{t}\left(\partial_{t} u_{n}(s)\right. & \left.-\Pi \Delta_{x} u_{n}(s), \phi^{2} u_{n}(s)-v_{n}(s)\right) \\
& -\left(\nabla_{x} u_{n}(s), \nabla_{x}\left(\phi^{2} u_{n}(s)\right)\right) d s
\end{aligned}
$$

Indeed, we have proved before that $u_{n} \rightarrow u$ in $C\left([0, T], L_{\varphi_{\mu . x_{0}}}^{2}(\Omega)\right)$. Since $u_{n}$ are uniformly bounded with respect to $n$ in the space $L^{\infty}\left([0, T], L_{b}^{2}(\Omega)\right)$ and $\|\theta\|_{L^{2}\left(\mathbb{R}^{1}\right)}<\infty$, then, due to Proposition 2.16, we establish that $u_{n} \rightarrow u$ in $C\left([0, T], L_{\theta}^{2}(\Omega)\right)$ and, analogously, $u_{n} \rightarrow u$ in $L^{2}\left([0, T], \mathcal{V}_{\theta}^{2}(\Omega)\right)$. Then, estimate (5.36) and Theorem 5.1 gives that

$$
v_{n} \rightarrow v \text { in } C\left([0, T], L_{\theta^{-1}}^{2}(\Omega)\right) \cap L^{2}\left([0, T], W_{\theta^{-1}}^{1,2}(\Omega)\right) .
$$

Thus, $R_{n}(t) \rightarrow R_{\infty}(t):=1 / 2\left(\phi^{2} u(t), u(t)\right)-(u(t), v(t))$ and we can pass to the limit in the left-hand side of (5.46). So, we only need to pass to the limit in the right-hand side of (5.46). The only nontrivial term there is the following one:

$$
\int_{0}^{t}\left|\left(\partial_{t} u_{n}(s), \phi^{2} u_{n}(s)-v_{n}(s)\right)-\left(\partial_{t} u(s), \phi^{2} u(s)-v(s)\right)\right| d s \rightarrow 0 \text { as } n \rightarrow \infty .
$$


The passing to the limit in the other terms is straightforward due to the above established convergences. In order to prove (5.48), we first note that $u_{n}$ are uniformly bounded in $\mathbb{W}_{b}([0, T] \times \Omega)$ and $u_{n} \rightarrow u$ in $\mathbb{W}_{\varphi_{\mu, x_{0}}}([0, T] \times \Omega)$. Thus, analogously to Proposition 2.16,

$$
\partial_{t} u_{n} \rightarrow \partial_{t} u, \quad \text { in } \quad L^{2}\left([0, T], \mathcal{H}_{\theta}^{-1,2}(\Omega)\right)+L^{4 / 3}\left([0, T], \mathcal{H}_{\theta^{3 / 2}}^{4 / 3}(\Omega)\right)
$$

(here we have used that $\left.\left\|\theta^{3 / 2}\right\|_{L^{4 / 3}(\mathbb{R})} \leq C\|\theta\|_{L^{2}(\mathbb{R})}^{3 / 2}<\infty\right)$. Thus, in order to verify the convergence (5.48), it is sufficient to check that

$$
\phi^{2} u_{n}-v_{n} \rightarrow \phi^{2} u-v \text { in } L^{2}\left([0, T], \mathcal{V}_{\theta^{-1}}^{2}(\Omega)\right) \cap L^{4}\left([0, T], \mathcal{H}_{\theta^{-3 / 2}}^{4}(\Omega)\right) .
$$

In order to verify (5.50), we recall that, due to Proposition 2.14 (analogously to (5.40)), the sequence $u_{n}$ is uniformly bounded in $L_{b}^{4}([0, T] \times \Omega)$ and, consequently, $u_{n} \rightarrow u$ in $L_{\theta^{1 / 2}}^{4}([0, T] \times \Omega)\left(\right.$ since $\left.\left\|\theta^{1 / 2}\right\|_{L^{4}(\Omega)}<\infty\right)$. Thus, $\phi^{2} u_{n} \rightarrow \phi^{2} u$ in $L_{\theta^{-3 / 2}}^{4}([0, T] \times \Omega)$ and, consequently,

$$
\phi^{2} u_{n} \rightarrow \phi^{2} u \text { in } L^{2}\left([0, T], W_{\theta^{-1}}^{1,2}(\Omega)\right) \cap L^{4}\left([0, T], L_{\theta^{-3 / 2}}^{4}(\Omega)\right)
$$

(we cannot write $\mathcal{V}_{\theta^{-1}}^{2}$ here $\operatorname{since} \operatorname{div}\left(\phi^{2} u_{n}\right) \neq 0$, but the vector field $\phi^{2} u-v$ is divergent free, so we will automatically obtain (5.50) if we verify separately the convergence of $\phi^{2} u_{n} \rightarrow \phi^{2} u$ and $v_{n} \rightarrow v$ in the space (5.51)).

So, we now need to establish this convergence for $v_{n}$. We also recall that the convergence in $L^{2}\left([0, T], W_{\theta^{-1}}^{1,2}(\Omega)\right)$ is already obtained in (5.47) and we only need to verify the convergence

$$
v_{n} \rightarrow v \text { in } L_{\theta^{-3 / 2}}^{4}([0, T] \times \Omega) .
$$

To this end, we note that the function $h_{n}=2 \phi \phi^{\prime} u_{n}$ in (5.30) is uniformly bounded in the space $L^{2}\left([0, T], W_{b, \theta^{-2}}^{1,2}(\Omega)\right) \cup L^{\infty}\left([0, T], L_{b, \theta^{-2}}^{2}(\Omega)\right)$ and, consequently, due to Theorem 5.1, the sequence $v_{n}$ is uniformly bounded in

$$
L^{\infty}\left([0, T], L_{b, \theta^{-2}}^{2}(\Omega)\right) \cap L^{2}\left([0, T], W_{b, \theta^{-2}}^{1,2}(\Omega)\right)
$$

and, due to Proposition 2.14, we also have that $v_{n}$ is uniformly bounded in the space $L_{b, \theta^{-2}}^{4}([0, T] \times \Omega)$. Moreover, due to (5.47) and Proposition 2.14, we have also the convergence $v_{n} \rightarrow v$ in $L_{\theta^{-1}}^{4}([0, T] \times \Omega)$. Therefore, since $\theta^{-2} \cdot \theta^{1 / 2}=\theta^{-3 / 2}$ and $\left\|\theta^{1 / 2}\right\|_{L^{4}(\mathbb{R})}<\infty$, this, together with Proposition 2.16, give the convergence $v_{n} \rightarrow v$ in $L_{\theta^{-3 / 2}}^{4}([0, T] \times \Omega)$. Thus, convergence (5.52) is verified and, consequently, the convergences (5.50) and (5.48) are also verified. Passing now to the limit $n \rightarrow \infty$ in (5.46), we finally verify that

$$
\begin{aligned}
& 1 / 2\left(\phi^{2} u(t), u(t)\right)-(u(t), v(t))-1 / 2\left(\phi^{2} u(0), u(0)\right)+(u(0), v(0)) \\
& =\int_{0}^{t}\left(\partial_{t} u_{n}(s)-\Pi \Delta_{x} u_{n}(s), \phi^{2} u_{n}(s)-v_{n}(s)\right)-\left(\nabla_{x} u_{n}(s), \nabla_{x}\left(\phi^{2} u_{n}(s)\right)\right) d s
\end{aligned}
$$

which is an integral equivalent of (5.32). Theorem 5.6 is proved. 
6. Nonlinear NS equations: a priori estimate. The aim of this section is to obtain a weighted energy estimate for the solutions of the Navier-Stokes equation

$$
\begin{aligned}
& \partial_{t} u+\left(u, \nabla_{x}\right) u=\Delta_{x} u-\nabla_{x} p+g, \\
& \left.u\right|_{\partial \Omega}=0, \quad \operatorname{div} u=0, \\
& \left.u\right|_{t=0}=u_{0}
\end{aligned}
$$

in a strip $\Omega$. Moreover, this problem is endowed by the natural additional flux assumption

$$
\mathrm{Su}_{1}(t) \equiv c
$$

where $c$ is a given constant which plays the role of a "boundary" condition at $x_{1}= \pm \infty$.

For simplicity we start our consideration with the case of zero flux

$$
\mathrm{S} u_{1}(t) \equiv 0
$$

and the case of general flux $c$ will be considered at the end of this section. We assume also that

$$
g \in L_{b}^{2}\left(\mathbb{R}_{+}, L_{b}^{2}(\Omega)\right), \quad u_{0} \in \mathcal{H}_{b}^{2}(\Omega)
$$

and the solution $u$ satisfies

$$
u \in \mathbb{W}_{b}([0, T] \times \Omega)
$$

(see Definition 5.5), and satisfies equation (6.1) in the sense of distributions $\mathcal{D}_{\text {div }}^{\prime}(\Omega)$ over the divergent free vector fields.

REMARK 6.1. Due to Theorem 5.6, $u \in L^{\infty}\left([0, T], \mathcal{H}_{b}^{2}(\Omega)\right) \cap C\left([0, T], \mathcal{H}_{\phi}^{2}(\Omega)\right)$ for every square integrable weight function of exponential growth rate, so the initial condition $\left.u\right|_{t=0}=u_{0}$ is well-defined. Moreover, since $u \in L^{\infty}\left([0, T], L_{b}^{2}(\Omega)\right) \cap$ $L_{b}^{2}\left([0, T], \mathcal{V}_{b}^{2}(\Omega)\right)$ then, due to the interpolation inequality of Proposition 2.14 (analogously to (5.40)), we have

$$
u \in L_{b}^{4}\left([0, T], \mathcal{H}_{b}^{4}(\Omega)\right) .
$$

Then, due to the Hölder inequality, the inertial term $\left(u, \nabla_{x} u\right)$ satisfies

$$
\begin{aligned}
\left\|\left(u, \nabla_{x}\right) u\right\|_{L_{b}^{4 / 3}([0, T] \times \Omega)} & \leq\left\||u| \cdot\left|\nabla_{x} u\right|\right\|_{L_{b}^{4 / 3}([0, T] \times \Omega)} \\
& \leq C\|u\|_{L_{b}^{4}([0, T] \times \Omega)}\left\|\nabla_{x} u\right\|_{L_{b}^{2}([0, T] \times \Omega)}
\end{aligned}
$$

and, consequently, $\left(u, \nabla_{x} u\right) \in L_{b}^{4 / 3}([0, T] \times \Omega)$. Theorem 4.4 now implies that

$$
\Pi\left[\left(u, \nabla_{x}\right) u\right] \in L_{b}^{4 / 3}\left([0, T], \mathcal{H}_{b}^{4 / 3}(\Omega)\right)
$$

(where $\Pi$ is the projector on the divergent free vector fields introduced in Section 4). Thus, applying this projector to equation (6.1), we obtain

$$
\partial_{t} u=\Pi \Delta_{x} u-\Pi\left[\left(u, \nabla_{x}\right) u\right]+\Pi g
$$


which shows that, indeed, the derivative $\partial_{t} u$ should belong to the space:

$$
\partial_{t} u \in L_{b}^{2}\left([0, T], \mathcal{H}_{b}^{-1,2}(\Omega)\right)+L_{b}^{4 / 3}\left([0, T], \mathcal{H}_{b}^{4 / 3}(\Omega)\right)
$$

(see Proposition 4.11 for the term $\Pi \Delta_{x} u$ ). This shows that the definition of a solution $u$ in the form (6.5) is not contradictive and equation (6.1) can be understood as equality (6.9) in the space (6.10). We also note that zero flux assumption (6.3) is now incorporated into the definition of the space $\mathbb{W}_{b}([0, T] \times \Omega)$.

We now introduce a special family of polynomial weight functions $\theta_{\varepsilon}(s)=\theta_{\varepsilon, x_{0}}(s)$ by the following expression:

$$
\theta_{\varepsilon, x_{0}}(s):=\left(1+\varepsilon^{2}\left|s-x_{0}\right|^{2}\right)^{-1 / 2}, \quad \varepsilon>0, \quad s, x_{0} \in \mathbb{R} .
$$

Obviously these functions are weight functions of exponential growth rate $\mu$, for every $\mu>0$ with the constant $C_{\theta_{\varepsilon}}$ depending on $\mu$, but independent of $x_{0} \in \Omega$ and $\varepsilon \in[0,1]$. This means that all of the weighted estimates formulated in previous sections will hold for weights (6.11) with the constants independent of $\varepsilon \rightarrow 0$ which is crucial for our method. Moreover, these weights also satisfy the following improved version of (5.28):

$$
\left|\phi_{\varepsilon, x_{0}}^{\prime}(s)\right| \leq \varepsilon\left[\phi_{\varepsilon, x_{0}}(s)\right]^{2},\left\|\phi_{\varepsilon}\right\|_{L^{2}\left(\mathbb{R}^{n}\right)}<\infty .
$$

Thus, Theorem 5.6 holds for these weights as well. The next proposition gives basic a priori estimate for the solutions of (6.1).

PROPOSITION 6.2. Let the above assumptions hold and let $u \in \mathbb{W}_{b}([0, T] \times \Omega)$ be a solution of the Navier-Stokes problem (6.1). Then, the following estimate holds:

$$
\begin{aligned}
& \sup _{s \in[0, T]}\left\{e^{-\alpha|t-s|}\|u(s)\|_{L_{\theta_{\varepsilon}}^{2}(\Omega)}^{2}\right\}+\left(C_{1}-C_{2} \varepsilon\|u\|_{L^{\infty}\left([0, T], L_{\theta_{\varepsilon}}^{2}(\Omega)\right)}\right) \times \int_{0}^{T} e^{-\alpha|t-s|}\|u(s)\|_{W_{\theta_{\varepsilon}}^{1,2}(\Omega)}^{2} d s \\
& \quad \leq C_{3} e^{-\alpha t}\|u(0)\|_{L_{\theta_{\varepsilon}}^{2}(\Omega)}^{2}+C_{3} \int_{0}^{T} e^{-\alpha|t-s|}\|g(s)\|_{L_{\theta_{\varepsilon}}^{2}(\Omega)}^{2} d s
\end{aligned}
$$

where the positive constants $\alpha$ and $C_{i}, i=1,2,3$ are independent of $u, u_{0}, g, \varepsilon \rightarrow 0, T$ and $x_{0}$ (we recall that we write for brevity $\theta_{\varepsilon}$ instead of $\theta_{\varepsilon, x_{0}}$ ).

Proof. Indeed, let $u$ be a solution of (6.9) belonging to the above class. Then, due to Theorem 5.6, we have the following identity:

$$
\begin{aligned}
& \frac{d}{d t}\left[1 / 2\left(\theta_{\varepsilon}^{2} u(t), u(t)\right)-(u(t), v(t))\right]+\left(\nabla_{x} u(t), \nabla_{x}\left(\theta_{\varepsilon}^{2} u(t)\right)\right) \\
& \quad=-\left(\theta_{\varepsilon}^{2} u(t)-v(t),\left(u(t), \nabla_{x}\right) u(t)-g(t)\right)
\end{aligned}
$$

where $v:=\mathbb{P}_{\theta_{\varepsilon}} u$ solves the auxiliary problem (5.30). Using (6.12) and the inequality $\|u\|_{L_{\theta_{\varepsilon}}^{2}(\Omega)} \leq\left\|\nabla_{x} u\right\|_{L_{\theta_{\varepsilon}}^{2}(\Omega)}$, we transform (6.14) as follows:

$$
\begin{aligned}
& \frac{d}{d t} R_{u}(t)+\alpha R_{u}(t)+1 / 2\|u(t)\|_{W_{\theta_{\varepsilon}}^{1,2}(\Omega)}^{2} \leq \mid\left(\theta_{\varepsilon}^{2} u(t),\left(u(t), \nabla_{x} u(t)\right) \mid\right. \\
& \quad+\left|\left(v(t),\left(u(t), \nabla_{x}\right) u(t)\right)\right|+C\|g(t)\|_{L_{\theta_{\varepsilon}}^{2}(\Omega)}^{2}+C\|v\|_{L_{\left[\theta_{\varepsilon}\right]^{-1}}^{2}(\Omega)}^{2}:=H_{u}(t)
\end{aligned}
$$


where $R_{u}(t):=1 / 2\|u(t)\|_{L_{\theta_{\varepsilon}}^{2}(\Omega)}^{2}-(u(t), v(t))$. Applying now the Gronwall inequality to (6.15), we infer

$$
R_{u}(t)+\int_{0}^{t} e^{-\alpha(t-s)}\|u(s)\|_{W_{\theta_{\varepsilon}}^{1,2}(\Omega)}^{2} d s \leq C e^{-\alpha t} R_{u}(0)+C \int_{0}^{t} e^{-\alpha(t-s)} H_{u}(s) d s .
$$

We now need to estimate the auxiliary function $v(t)$. To this end, we note that, due to (6.11), the function $h_{u}(t):=2 \theta_{\varepsilon} \theta_{\varepsilon}^{\prime} u(t)$ satisfies

$$
\left\|h_{u}(t)\right\|_{W_{\left[\theta_{\varepsilon}\right]^{-2}}^{l, 2}(\Omega)} \leq C \varepsilon\|u(t)\|_{W_{\theta_{\varepsilon}}^{l, 2}(\Omega)}
$$

where the constant $C$ is independent of $\varepsilon \rightarrow 0$. Applying now Theorem 5.1 to the auxiliary equation (5.30), we deduce the following estimates:

$$
\begin{gathered}
\|v(t)\|_{W_{\left[\theta_{\varepsilon}\right]^{-2}}^{1,2}(\Omega)}^{2} \leq C \varepsilon^{2}\|u(t)\|_{L_{\theta_{\varepsilon}}^{2}(\Omega)}^{2}+C \varepsilon^{2} \int_{0}^{T} e^{-\alpha|t-s|}\|u(s)\|_{W_{\theta_{\varepsilon}}^{1,2}(\Omega)}^{2} d s, \\
\int_{0}^{T} e^{-\alpha|t-s|}\|v(s)\|_{W_{\left[\theta_{\varepsilon}\right]^{2}}^{2,2}(\Omega)}^{2} d s \leq C \varepsilon^{2} \int_{0}^{T} e^{-\alpha|t-s|}\|u(s)\|_{W_{\theta_{\varepsilon}}^{1,2}(\Omega)}^{2} d s
\end{gathered}
$$

where $\alpha>0$ is small enough and the constants $C$ and $\alpha$ are independent of $\varepsilon \rightarrow 0$. Inserting these estimates into (6.16) and arguing analogously to (5.38), we get

$$
\begin{aligned}
\|u(t)\|_{L_{\theta_{\varepsilon}}^{2}(\Omega)}^{2} & +\int_{0}^{t} e^{-\alpha(t-s)}\|u(s)\|_{W_{\theta_{\varepsilon}}^{1,2}(\Omega)}^{2} d s \leq C e^{-\alpha t}\left\|u_{0}\right\|_{L_{\theta_{\varepsilon}}^{2}(\Omega)}^{2} \\
& +C \varepsilon^{2} \int_{0}^{T} e^{-\alpha|t-s|}\|u(s)\|_{W_{\theta_{\varepsilon}}^{1,2}(\Omega)}^{2} d s+\int_{0}^{T} e^{-\alpha|t-s|} H_{u}(s) d s .
\end{aligned}
$$

This estimate, in turn, implies in a standard way that, for sufficiently small $\varepsilon>0$,

$$
\begin{aligned}
& \sup _{s \in[0, T]}\left\{e^{-\alpha|t-s|}\|u(t)\|_{L_{\theta_{\varepsilon}}^{2}(\Omega)}^{2}\right\}+C^{\prime} \int_{0}^{T} e^{-\alpha|t-s|}\|u(s)\|_{W_{\theta_{\varepsilon}}^{1,2}(\Omega)}^{2} d s \\
& \leq C e^{-\alpha t}\left\|u_{0}\right\|_{L_{\theta_{\varepsilon}}^{2}(\Omega)}^{2}+C \int_{0}^{T} e^{-\alpha|t-s|}\|g(s)\|_{L_{\theta_{\varepsilon}}^{2}(\Omega)}^{2} d s \\
& \quad+C \int_{0}^{T} e^{-\alpha|t-s|}\left|\left(\theta_{\varepsilon}^{2} u(s),\left(u(s), \nabla_{x}\right) u(s)\right)\right| d s \\
& \quad+C \int_{0}^{T} e^{-\alpha|t-s|}\left|\left(v(s),\left(u(s), \nabla_{x}\right) u(s)\right)\right| d s:=I_{u_{0}}+I_{g}+I_{1}+I_{2} .
\end{aligned}
$$

Indeed, in order to obtain the estimate for the first term in the left-hand side of (6.20), it is sufficient to multiply (6.19) by $e^{-\beta\left|t_{1}-t\right|}$, where $\beta<\alpha$, take the supremum over $t \in[0, T]$ and use Proposition 2.5. Analogously, in order to obtain the estimate for the second term, we only need to integrate over $t \in[0, T]$ instead of taking the supremum (rigorously speaking, we obtain (6.20) for some new exponent $\beta$ which is less than $\alpha$ (say, $\beta=\alpha / 2$ ), but, in order to simplify the notations, we denote this new exponent by $\alpha$ as well.

Thus, in order to finish the proof of Proposition 6.2, we only need to estimate the integrals $I_{1}$ and $I_{2}$ in the right-hand side of (6.20). To this end, we note that, integrating 
by parts in the term $\left(\theta_{\varepsilon}^{2} u,\left(u, \nabla_{x}\right) u\right)$ and using $\operatorname{div} u=0$ and inequality (6.12), we have

$$
\begin{aligned}
\left|\left(\theta_{\varepsilon}^{2} u,\left(u, \nabla_{x}\right) u\right)\right| & =\left|\left(2 \theta_{\varepsilon} \theta_{\varepsilon}^{\prime} u,|u|^{2}\right)\right| \leq C \varepsilon\left(\left[\theta_{\varepsilon}\right]^{3}|u|,|u|^{2}\right) \\
& \leq C_{1} \varepsilon\|u\|_{L_{\theta_{\varepsilon}}^{2}(\Omega)}\|u\|_{L_{\theta_{\varepsilon}}^{4}(\Omega)}^{2} \leq C_{2} \varepsilon\|u\|_{L_{\theta_{\varepsilon}}^{2}(\Omega)}\|u\|_{W_{\theta_{\varepsilon}}^{1,2}(\Omega)}^{2}
\end{aligned}
$$

where the constant $C_{2}$ is independent of $\varepsilon$ (here we have implicitly used also the embedding $W_{\theta_{\varepsilon}}^{1,2}(\Omega) \subset L_{\theta_{\varepsilon}}^{4}(\Omega)$ where the embedding constant is independent of $\varepsilon$; see Proposition 2.10).

Inserting this estimate into the expression for $I_{1}$, we arrive at

$$
I_{1} \leq C_{3} \varepsilon\|u\|_{L^{\infty}\left([0, T], L_{\theta_{\varepsilon}}^{2}(\Omega)\right)} \int_{0}^{T} e^{-\alpha|t-s|}\|u(s)\|_{W_{\theta_{\varepsilon}}^{1,2}(\Omega)}^{2} d s .
$$

Let us now estimate the integral $I_{2}$. To this end we will use the following embedding estimate of Proposition 2.10:

$$
\|v\|_{L_{\left[\theta_{\varepsilon}\right]^{-2}}^{\infty}(\Omega)} \leq C\|v\|_{W_{\left[\theta_{\varepsilon}\right]^{-2}}^{2,2}(\Omega)}
$$

where again the constant $C$ is independent of $\varepsilon$. Thus, we can estimate the term $I_{2}$ as follows:

$$
\begin{aligned}
I_{2} & \leq C \int_{0}^{T} e^{-\alpha|t-s|}\|u(s)\|_{L_{\theta_{\varepsilon}}^{2}(\Omega)}\left\|\nabla_{x} u(s)\right\|_{L_{\theta_{\varepsilon}}^{2}(\Omega)}\|v(s)\|_{W_{\left[\theta_{\varepsilon}\right]^{-2}}^{2,2}(\Omega)} d s \\
& \leq C\|u(s)\|_{L^{\infty}\left([0, T], L_{\theta_{\varepsilon}}^{2}(\Omega)\right)} \int_{0}^{T} e^{-\alpha|t-s|}\left(\varepsilon\|u(t)\|_{W_{\theta_{\varepsilon}}^{1,2}(\Omega)}^{2}+\varepsilon^{-1}\|v(s)\|_{W_{\left[\theta_{\varepsilon}\right]^{-2}}^{2,2}(\Omega)}^{2}\right) d s .
\end{aligned}
$$

Using now (6.18), we finally arrive at

$$
I_{2} \leq C_{3} \varepsilon\|u\|_{L^{\infty}\left([0, T], L_{\theta_{\varepsilon}}^{2}(\Omega)\right)} \int_{0}^{T} e^{-\alpha|t-s|}\|u(s)\|_{W_{\theta_{\varepsilon}}^{1,2}(\Omega)}^{2} d s .
$$

Inserting estimates (6.22) and (6.24) into the right-hand side of (6.20), we obtain (6.13) and finish the proof of Proposition 6.2.

In order to deduce the existence of a solution $u \in \mathbb{W}_{b}([0, T] \times \Omega)$ of problem (6.1) from a priori estimate (6.13), we need the following simple proposition.

Proposition 6.3. Let $w \in L_{b}^{2}(\Omega)$ and let the weight $\theta_{\varepsilon}=\theta_{\varepsilon, x_{0}}$ be the weight function defined by (6.11). Then, the following estimate holds:

$$
\|w\|_{L_{\theta_{\varepsilon}}^{2}(\Omega)} \leq C \varepsilon^{-1 / 2}\|w\|_{L_{b}^{2}(\Omega)}
$$

where the constant $C$ is independent of $\varepsilon \rightarrow 0$ and $x_{0} \in \mathbb{R}$.

Proof. Indeed, according to (2.11), we have

$$
\begin{aligned}
\|w\|_{L_{\theta_{\varepsilon}}^{2}(\Omega)}^{2} & \leq C \int_{s \in \mathbb{R}} \theta_{\varepsilon}(s)^{2}\|w\|_{L^{2}\left(\Omega_{s}\right)}^{2} d s \leq C\|w\|_{L_{b}^{2}(\Omega)}^{2} \int_{s \in \mathbb{R}}\left(1+\varepsilon^{2}\left|s-x_{0}\right|^{2}\right)^{-1} d s \\
& =C\|w\|_{L_{b}^{2}(\Omega)}^{2} \varepsilon^{-1} \int_{s \in \mathbb{R}}\left(1+|s|^{2}\right)^{-1} d s=C_{1} \varepsilon^{-1}\|w\|_{L_{b}^{2}(\Omega)}^{2}
\end{aligned}
$$

and Proposition 6.3 is proved. 
Proposition 6.3 allows us to simplify basic a priori estimate (6.13) as follows.

COROLlary 6.4. Let the assumptions of Proposition 6.2 hold and let $u \in \mathbb{W}_{b}([0, T] \times$ $\Omega)$ be a solution of (6.1). Then, the following estimate holds:

$$
\begin{aligned}
& \|u\|_{L^{\infty}\left([0, T], L_{\theta_{\varepsilon}}^{2}(\Omega)\right)}^{2}+\left(C_{1}-C_{2} \varepsilon\|u\|_{L^{\infty}\left([0, T], L_{\theta_{\varepsilon}}^{2}(\Omega)\right)}\right)\|u\|_{L_{b}^{2}\left([0, T], W_{\theta_{\varepsilon}}^{1,2}(\Omega)\right)}^{2} \\
& \quad \leq C_{3} \varepsilon^{-1}\left(\|u(0)\|_{L_{b}^{2}(\Omega)}^{2}+\|g\|_{L_{b}^{2}\left([0, T], L_{b}^{2}(\Omega)\right)}^{2}\right)
\end{aligned}
$$

where the positive constants $\alpha$ and $C_{i}, i=1,2,3$ are independent of $u, u_{0}, g, \varepsilon \rightarrow 0, T$ and $x_{0}$ (we recall that we write for brevity $\theta_{\varepsilon}$ instead of $\theta_{\varepsilon, x_{0}}$ ).

Indeed, in order to deduce (6.26) from (6.13), it is sufficient to use (6.25), take the supremum over $t \in[0, T]$ and use (2.13).

We are now ready to prove the existence of a bounded solution of the Navier-Stokes problem (6.1).

THEOREM 6.5. Let the above assumptions hold. Then problem (6.1) possesses at least one solution $u \in \mathbb{W}_{b}([0, T] \times \Omega)$ which satisfies the following estimate:

$$
\|u\|_{L^{\infty}\left([0, T], L_{b}^{2}(\Omega)\right) \cap L_{b}^{2}\left([0, T], W_{b}^{1,2}(\Omega)\right)} \leq C\left(1+\left\|u_{0}\right\|_{L_{b}^{2}(\Omega)}^{2}+\|g\|_{L_{b}^{2}([0, T] \times \Omega)}^{2}\right)
$$

where the constant $C$ is independent of $T, g$ and $u_{0}$.

Proof. The idea of the proof is based on the following observation: let

$$
K_{u_{0}, g}:=\left(1+\left\|u_{0}\right\|_{L_{b}^{2}(\Omega)}^{2}+\|g\|_{L_{b}^{2}([0, T] \times \Omega)}^{2}\right)^{1 / 2} .
$$

Then, a priori estimate (6.26) gives the following conditional result: let the solution $u$ a priori satisfy

$$
\|u\|_{L^{\infty}\left([0, T], L_{\theta_{\varepsilon}}^{2}(\Omega)\right)} \leq \frac{C_{1}}{2 C_{2} \varepsilon} .
$$

Then, we necessarily have

$$
\|u\|_{L^{\infty}\left([0, T], L_{\theta_{\varepsilon}}^{2}(\Omega)\right)}+C_{1} / 2\|u\|_{L_{b}^{2}\left([0, T], W_{\theta_{\varepsilon}}^{1,2}(\Omega)\right)} \leq C_{3}^{1 / 2} \varepsilon^{-1 / 2} K_{u_{0}, g}
$$

Let us now fix $\varepsilon \ll 1$ in such way that

$$
C_{3}^{1 / 2} \varepsilon^{-1 / 2} K_{g, u_{0}}<\frac{C_{1}}{2 C_{2} \varepsilon}
$$

or, which is the same,

$$
\varepsilon \sim\left[K_{u_{0}, g}\right]^{-2} .
$$

In this case estimates (6.29) and (6.30) allow us to deduce estimate of the form (6.27) using the standard continuation by parameter arguments. Indeed, let $u^{s}, s \in[0,1]$ be a continuous curve of solutions of (6.1) such that

$$
K_{u_{0}^{s}, g^{s}} \leq K_{u_{0}^{1}, g^{1}}
$$


and estimate (6.30) is satisfied for $s=0$. Then it is satisfied for $s=1$ as well, since, due to (6.31), we cannot achieve the bound (6.29) before crossing the bound (6.30) and, consequently, the continuity arguments show that (6.30) holds for every $s \in[0,1]$.

Let us now proceed in more rigorous way. To this end, we first prove estimate (6.27) for the square integrable case:

$$
u_{0} \in \mathcal{H}^{2}(\Omega), \quad g \in L^{2}\left([0, T], L^{2}(\Omega)\right) .
$$

It is well-known that, in this case, the Navier-Stokes problem has a unique square integrable solution $u$ :

$$
u \in C\left([0, T], L^{2}(\Omega)\right) \cap L^{2}\left([0, T], W^{1,2}(\Omega)\right) .
$$

Moreover, this solution depends continuously (in the metric of (6.35)) on the initial data $u_{0}$ and external forces $g$; see e.g. [4], [5], [24].

Thus, the solutions $u^{s}, s \in[0,1]$, associated with the initial data $u_{0}^{s}:=s u_{0}, g^{s}:=s g$, generate a continuous curve in the space (6.35) and, evidently, (6.30) is satisfied for $u^{0} \equiv 0$. Therefore, due to the above continuity arguments, we have estimate (6.30) for $s=1$ as well. Taking into account (6.32), we can rewrite it in the following way:

$$
\|u\|_{L^{\infty}\left([0, T], L_{\theta_{\varepsilon, x_{0}}}^{2}(\Omega)\right) \cap L_{b}^{2}\left([0, T], W_{\theta_{\varepsilon, x_{0}}}^{1,2}(\Omega)\right)} \leq C\left[K_{u_{0}, g}\right]^{2}
$$

where the constant $C$ is independent of $x_{0} \in \mathbb{R}$. Using now the obvious estimate

$$
\|v\|_{W_{b}^{l, 2}(\Omega)} \leq C \sup _{x_{0} \in \mathbb{R}}\|v\|_{W_{\theta_{\varepsilon, x_{0}}}^{l, 2}(\Omega)}, \quad l=0,1
$$

where $C$ is independent of $\varepsilon \ll 1$, we deduce the required estimate (6.27).

Thus, the assertion of the theorem is verified in the square integrable case (6.34). Let us now consider the general case of $u_{0}$ and $g$ satisfying only assumption (6.4). To this end, we approximate the data $u_{0}$ and $g$ by a sequence of square integrable ones $u_{0}^{n}$ and $g^{n}$ satisfying (6.34). Moreover, we assume that

$$
\left\|u_{0}^{n}\right\|_{\mathcal{H}_{b}^{2}(\Omega)}+\left\|g^{n}\right\|_{L_{b}^{2}([0, T] \times \Omega)} \leq C
$$

where $C$ is independent of $n$ and that

$$
u_{0}^{n} \rightarrow u_{0} \text { in } L_{l o c}^{2}(\bar{\Omega}), \quad g^{n} \rightarrow g \text { in } L_{l o c}^{2}([0, T] \times \bar{\Omega}) .
$$

Then, due to already proven part of estimate (6.27), the associated solution $u^{n}$ of the Navier-Stokes equation (belonging to the class (6.35)) satisfies

$$
\left\|u^{n}\right\|_{L^{\infty}\left([0, T], L_{b}^{2}(\Omega)\right)}+\left\|u^{n}\right\|_{L_{b}^{2}\left([0, T], W_{b}^{1,2}(\Omega)\right)}+\left\|u_{n}\right\|_{L_{b}^{4}([0, T] \times \Omega)} \leq C_{1}
$$

where $C_{1}$ is also independent of $n$. Moreover, from equation (6.9), we infer also that

$$
\left\|\partial_{t} u^{n}\right\|_{L_{b}^{2}\left([0, T], \mathcal{H}_{b}^{-1,2}(\Omega)\right)+L_{b}^{4 / 3}\left([0, T], \mathcal{H}_{b}^{4 / 3}(\Omega)\right)} \leq C .
$$

Thus, passing to the subsequence if necessary, we can assume without loss of generality that the sequence $u^{n}$ converges weakly to some $u \in \mathbb{W}_{b}([0, T] \times \Omega)$ in the local topology, i.e., for every square integrable weight $\phi$ satisfying (5.28), we have

$$
u^{n} \rightarrow u \text { weakly in } \mathbb{W}_{\phi}([0, T] \times \Omega) .
$$


Moreover, due to the embedding $\mathbb{W}_{\phi}([0, T] \times \Omega) \subset C\left([0, T], L_{\phi}^{2}(\Omega)\right)$ (which is actually proved in Theorem 5.6), the limit function $u$ satisfies the initial condition $u(0)=u_{0}$.

Thus, we only need to verify that the constructed function $u$ satisfies equation (6.1) (or which is the same, equation (6.9)) in the sense of distributions, i.e., we need to verify that, for every $w \in C_{0}^{\infty}((0, T) \times \Omega)$ with $\operatorname{div} w=0$, we have

$$
-\left\langle u, \partial_{t} w\right\rangle=\left\langle u, \Delta_{x} w\right\rangle-\left\langle\left(u, \nabla_{x}\right) u, w\right\rangle+\langle g, w\rangle .
$$

Indeed, since $u^{n}$ solves the Navier-Stokes equations, we have

$$
-\left\langle u^{n}, \partial_{t} w\right\rangle=\left\langle u^{n}, \Delta_{x} w\right\rangle-\left\langle\left(u^{n}, \nabla_{x}\right) u^{n}, w\right\rangle+\left\langle g^{n}, w\right\rangle .
$$

Moreover, passing to the limit $n \rightarrow \infty$ in all linear terms of (6.43) is evident and we only need to pass to the limit in the inertial term $\left(u^{n}, \nabla_{x}\right) u^{n}$. To this end, it is sufficient to verify that

$$
u^{n} \rightarrow u \text { strongly in the space } L_{l o c}^{2}([0, T] \times \bar{\Omega}) .
$$

Indeed, since $\nabla_{x} u^{n} \rightarrow \nabla_{x} u$ weakly in $L_{l o c}^{2}([0, T] \times \bar{\Omega})$, then (6.44) implies the weak convergence $\left(u^{n}, \nabla_{x}\right) u^{n} \rightarrow\left(u, \nabla_{x}\right) u$ in $L_{l o c}^{1}([0, T] \times \bar{\Omega})$.

In order to prove (6.44), we note that $\mathcal{H}_{b}^{4 / 3}(\Omega) \subset \mathcal{H}_{b}^{-1,2}(\Omega)$ and, consequently, for every square integrable weight function $\phi$, we have

$$
\partial_{t} u^{n} \rightarrow \partial_{t} u \text { weakly in } L^{4 / 3}\left([0, T], \mathcal{H}_{\phi^{2}}^{-1,2}(\Omega)\right)
$$

Furthermore, due to (6.41), we have also

$$
u^{n} \rightarrow u \text { weakly in } L^{2}\left([0, T], \mathcal{V}_{\phi}^{2}(\Omega)\right)
$$

Since we have the standard embeddings

$$
\mathcal{V}_{\phi}^{2}(\Omega) \subset \subset \mathcal{H}_{\phi^{2}}^{2}(\Omega) \subset \mathcal{H}_{\phi^{2}}^{-1,2}(\Omega)
$$

and the first embedding is compact, then, due to the compactness theorem (see e.g. [23]), we have the strong convergence $u^{n} \rightarrow u$ in $L^{2}\left([0, T], \mathcal{H}_{\phi^{2}}^{2}(\Omega)\right)$. Thus, the convergence (6.44) is proved and Theorem 6.5 is also proved.

We now return to the general case of nonzero flux $c \neq 0$ in (6.2). Then the NavierStokes equation (6.1) with $g=0$ possesses the classical Poiseuille solution

$$
v_{c}(x):=\left(\frac{3}{2} c\left(1-x_{2}^{2}\right), 0\right) .
$$

Obviously, if (6.2) is satisfied, then the difference $u-v_{c}$ has zero flux and, consequently, it is natural to define a weak solution of (6.1) as a function $u \in v_{c}+\mathbb{W}_{b}([0, T] \times \Omega)$ which satisfies (6.1) in the sense of distributions over the divergent free vector fields. Moreover, the assumption on $u_{0}$ should be also naturally replaced by

$$
u_{0} \in v_{c}+\mathcal{H}_{b}^{2}(\Omega)
$$

The next theorem is an analogue of Theorem 6.5 for the case of nonzero flux. 
THEOREM 6.6. Let the above assumptions hold. Then, for every $c \in \mathbb{R}, u_{0} \in v_{c}+$ $\mathcal{H}_{b}^{2}(\Omega)$ and $g \in L_{b}^{2}([0, T] \times \Omega)$, the Navier-Stokes problem (6.1), (6.2) possesses at least one weak solution $u \in v_{c}+\mathbb{W}_{b}([0, T] \times \Omega)$ which satisfies the following estimate:

$$
\|u\|_{L^{\infty}\left([0, T], L_{b}^{2}(\Omega)\right) \cap L_{b}^{2}\left([0, T], W_{b}^{1,2}(\Omega)\right)} \leq C\left(1+c^{3}+\left\|u_{0}\right\|_{L_{b}^{2}(\Omega)}^{2}+\|g\|_{L_{b}^{2}([0, T] \times \Omega)}^{2}\right)
$$

where the constant $C$ is independent of $T, u_{0}, g$ and $c$.

Proof. We want to reduce the general case to the particular case of zero flux considered above. The most natural way to do so is to make the variable change $\bar{u}:=u-v_{c}$ where the $v_{c}$ is the Poiseuille flow, but this scheme does not work, since the Poiseuille flow can be unstable. Instead of this, we construct below some special solution of the stationary Navier-Stokes problem $(6.1),(6.2)$ of the form $V_{c}(x):=\left(V_{c}\left(x_{2}\right), 0\right)$, $V_{c}( \pm 1)=0$ (with the appropriate nonzero external force $g_{c}$ ) and introduce a new unknown $\bar{u}:=u-V_{c}$. Then, this function belongs to $\mathbb{W}_{b}([0, T] \times \Omega)$ and solves

$$
\begin{aligned}
& \partial_{t} \bar{u}+\left(\bar{u}, \nabla_{x}\right) \bar{u}=\Delta_{x} \bar{u}+L_{V_{c}} \bar{u}-\nabla_{x} p+g-g_{c}, \\
& \operatorname{div} \bar{u}=0,\left.\quad \bar{u}\right|_{\partial \Omega}=0, \quad \mathbb{S} \bar{u}_{1} \equiv 0, \\
& \left.\bar{u}\right|_{t=0}=\bar{u}_{0}:=u_{0}-V_{c} .
\end{aligned}
$$

which differs from (6.1) by the presence of the additional linear operator $L_{V_{c}}$

$$
L_{V_{c}} w:=\left(V_{c}, \nabla_{x}\right) w+\left(w, \nabla_{x}\right) V_{c} .
$$

The next Lemma specifies the choice of the special function $V_{c}$.

Lemma 6.7. Let $c \in \mathbb{R}$ be arbitrary. Then there exist a vector field $V_{c}(x)=$ $\left(V_{c}\left(x_{2}\right), 0\right), V_{c}( \pm 1)=0$ such that

$$
\left(L_{V_{c}} w, w\right) \leq 1 / 2\|w\|_{W^{1,2}(\Omega)}^{2}, \quad \forall w \in W_{0}^{1,2}(\Omega)
$$

and

$$
\left\|V_{c}\right\|_{C([-1,1])} \leq \kappa|c|, \quad\left\|V_{c}^{\prime}\right\|_{L^{2}([-1,1])} \leq \kappa\left(|c|^{3 / 2}+|c|\right),
$$

where the constant $\kappa$ is independent of $c$ and $g_{c}=-V_{c}^{\prime \prime}$.

Proof. We seek the required function $V_{c}\left(x_{2}\right)$ in the following form:

$$
V_{c}(z)=\left\{\begin{array}{l}
\lambda, \quad z \in[-1+\delta, 1-\delta] \\
\lambda \delta^{-1}(1-z), \quad z \in[1-\delta, 1], \\
\lambda \delta^{-1}(1+z), \quad z \in[-1,-1+\delta]
\end{array}\right.
$$

where $\delta \ll 1$ is small positive constant and $\lambda$ is close to $c$. Obviously, in order to satisfy the flux condition, we need

$$
2 c=\int_{-1}^{1} V_{c}(z) d z=\lambda(2-2 \delta)+\lambda \delta=2 \lambda-\lambda \delta .
$$

So, we now need to fix $\delta$ in such a way that (6.51) will be satisfied. Indeed, let $w \in$ 
$\left[W_{0}^{1,2}(\Omega)\right]^{2}$. Then, direct calculation gives

$$
\begin{aligned}
\left(L_{V_{c}} w, w\right) & =\left(w_{2} \partial_{x_{2}} V_{c}, w_{1}\right)=\left(V_{c}^{\prime}, w_{2} w_{1}\right) \\
& \leq \lambda \delta^{-1} \int_{x_{1} \in \mathbb{R}}\left(\int_{-1}^{-1+\delta}\left|w\left(x_{1}, x_{2}\right)\right|^{2} d x_{2}+\int_{1-\delta}^{1}\left|w\left(x_{1}, x_{2}\right)\right|^{2} d x_{2}\right) d x_{1}
\end{aligned}
$$

We now recall that $w\left(x_{1}, \pm 1\right)=0$ and, consequently,

$$
\int_{-1}^{-1+\delta}\left|w\left(x_{1}, x_{2}\right)\right|^{2} d x_{2} \leq \delta^{2} \int_{-1}^{1}\left|\partial_{x_{2}} w\left(x_{1}, x_{2}\right)\right|^{2} d x_{2}
$$

and the analogous estimate holds near $x_{2}=1$. Thus,

$$
\left(L_{V_{c}} w, w\right) \leq 2 \lambda \delta\|w\|_{W_{0}^{1,2}(\Omega)}^{2}
$$

and we only need to satisfy the following conditions:

$$
2 c=2 \lambda-\lambda \delta, \quad 0<\delta \leq 1, \quad 2 \lambda \delta \leq 1 / 2 .
$$

These inequalities will be satisfied if we take, e.g., $\delta:=\min \{1,1 /(4|\lambda|)\}$ (and $\lambda$ is uniquely defined by $c$ from the first equation of (6.57). It is also not difficult to verify that the function $V_{c}$ thus defined also satisfies inequalities (6.52). Lemma 6.7 is proved.

We are now ready to finish the proof of Theorem 6.6. This proof repeats, with minor changes, the proof of Theorem 6.5 for the case of zero flux. The only difference is that we now have the additional linear term $L_{V_{c}} \bar{u}$ in equation (6.49) which is not essential due to estimate (6.51).

Indeed, proving the analogue of basic a priori estimate (6.13), we will only have the additional terms

$$
\left(L_{V_{c}} \bar{u}, \theta_{\varepsilon}^{2} \bar{u}\right)-\left(L_{V_{c}} \bar{u}, v\right)-\left(V_{c}^{\prime}, \partial_{x_{2}}\left(\theta_{\varepsilon}^{2} \bar{u}-v\right)\right)
$$

in the right-hand side of (6.15). The first term of (6.58) can be estimated using Lemma 6.7 as follows:

$$
\begin{aligned}
\left(L_{V_{c}} \bar{u}, \theta_{\varepsilon}^{2} \bar{u}\right) & =\left(L_{V_{c}}\left(\theta_{\varepsilon} \bar{u}\right), \theta_{\varepsilon} \bar{u}\right)-\left(V_{c}, \theta_{\varepsilon}^{\prime} \theta_{\varepsilon}|\bar{u}|^{2}\right) \\
& \leq 1 / 2\left\|\nabla_{x}\left(\theta_{\varepsilon} \bar{u}\right)\right\|_{L^{2}(\Omega)}^{2}-\kappa c \varepsilon\|u\|_{L_{\theta_{\varepsilon}}^{2}(\Omega)}^{2},
\end{aligned}
$$

where the constant $\kappa$ is independent of $\bar{u}, c$ and $\varepsilon$.

In order to estimate the second additional term of (6.58) we split it into a sum of two terms $\left(\left(V_{c}, \nabla_{x}\right) \bar{u}, v\right)$ and $\left(\left(\bar{u}, \nabla_{x}\right) V_{c}, v\right)$. The first of them can be easily estimated by (6.52) and Hölder inequality as

$$
\left|\left(\left(V_{c}, \nabla_{x}\right) \bar{u}, v\right)\right| \leq \kappa^{\prime} c\|\bar{u}\|_{W_{\theta_{\varepsilon}}^{1,2}(\Omega)}\|v\|_{L_{\left[\theta_{\varepsilon}\right]^{-1}}^{2}(\Omega)}
$$

and the second one can be estimated exactly as in (6.55), as follows:

$$
\left|\left(\left(\bar{u}, \nabla_{x}\right) V_{c}, v\right)\right| \leq \kappa\|\bar{u}\|_{W_{\theta_{\varepsilon}}^{1,2}(\Omega)}\|v\|_{W_{\left[\theta_{\varepsilon}\right]^{-1}}^{1,2}(\Omega)} .
$$

Thus, the second additional term is estimated as follows:

$$
\left|\left(L_{V_{c}} \bar{u}, v\right)\right| \leq \kappa(c+1)\|\bar{u}\|_{W_{\theta_{\varepsilon}}^{1,2}(\Omega)}\|v\|_{W_{\left[\theta_{\varepsilon}\right]^{-1}}^{1,2}(\Omega)},
$$

where the constant $\kappa$ is independent of $\varepsilon, c, u$ and $v$. 
Finally, the third additional term of (6.58) can be estimates using (6.52) and Hölder inequality:

$$
\begin{aligned}
\left|\left(V_{c}^{\prime}, \partial_{x_{2}}\left(\theta_{\varepsilon}^{2} \bar{u}-v\right)\right)\right| & \leq C_{\delta}\left\|V_{c}^{\prime}\right\|_{L_{\theta_{\varepsilon}}^{2}(\Omega)}^{2}+\delta\left(\|\bar{u}\|_{W_{\theta_{\varepsilon}}^{1,2}(\Omega)}^{2}+\|v\|_{W_{\left[\theta_{\varepsilon}\right]^{-1}}^{1,2}(\Omega)}^{2}\right) \\
& \leq \kappa_{\delta}\left(c^{3}+1\right) \varepsilon^{-1}+\delta\left(\|\bar{u}\|_{W_{\theta_{\varepsilon}}^{1,2}(\Omega)}^{2}+\|v\|_{W_{\left[\theta_{\varepsilon}\right]^{-1}}^{1,2}(\Omega)}^{2}\right)
\end{aligned}
$$

where $\delta>0$ is arbitrary and the constant $\kappa_{\delta}$ depends on $\delta$, but is independent of $c, \varepsilon$, $u$ and $v$.

Estimates (6.59)-(6.61) show that, under the additional assumption

$$
c \varepsilon \leq \kappa
$$

where $\kappa>0$ is a sufficiently small number independent of $c$ and $\varepsilon$ (we recall that, due to $(6.18), v \sim \varepsilon \bar{u})$, we can repeat word by word the proof of (6.13) and obtain the following analogue of (6.26):

$$
\begin{aligned}
& \|\bar{u}\|_{L^{\infty}\left([0, T], L_{\theta_{\varepsilon}}^{2}(\Omega)\right)}^{2}+\left(C_{1}-C_{2} \varepsilon\|\bar{u}\|_{L^{\infty}\left([0, T], L_{\theta_{\varepsilon}}^{2}(\Omega)\right)}\right)\|\bar{u}\|_{L_{b}^{2}\left([0, T], W_{\theta_{\varepsilon}}^{1,2}(\Omega)\right)}^{2} \\
& \quad \leq C_{3} \varepsilon^{-1}\left(1+c^{3}+\|\bar{u}(0)\|_{L_{b}^{2}(\Omega)}^{2}+\|g\|_{L_{b}^{2}\left([0, T], L_{b}^{2}(\Omega)\right)}^{2}\right)
\end{aligned}
$$

where the positive constants $\alpha$ and $C_{i}, i=1,2,3$ are independent of $u, u_{0}, g, \varepsilon \rightarrow 0$, $T, c$ and $x_{0}$.

Furthermore, arguing exactly as in the proof of estimate (6.27), we deduce a priori estimate (6.48) (see (6.28)-(6.32)). The existence of a solution can then be verified exactly as in the case of zero flux $c$. Theorem 6.6 is proved.

REMARK 6.8. Arguing analogously, it is not difficult to verify the existence of a solution of more general Navier-Stokes problem with the nonautonomous flux

$$
\mathrm{Su}_{1}(t) \equiv c(t)
$$

where $c \in C^{1}([0, T])$ is an arbitrary given function. Moreover, the assumption on the external force $g$ can be relaxed to

$$
g \in L_{b}^{2}\left([0, T], \mathcal{H}_{b}^{-1,2}(\Omega)\right)
$$

Furthermore, the weighted theory developed in this section allows to consider not only bounded with respect to $x_{1} \rightarrow \infty$ solutions, but also slowly growing solutions of the NS equation (growing not faster than $\left|x_{1}\right|^{1 / 2-\delta}, \delta>0$ is arbitrary). We however will not use these facts in the sequel and, for this reason, do not give their rigorous proofs here.

7. Nonlinear NS equations: uniqueness and regularity. In this section, we verify that the solution of the Navier-Stokes equations constructed in the previous section is unique and prove some smoothness of these solutions. We start with the following theorem which gives the Lipschitz continuity of weak energy solution of the N-S equations with respect to the initial data. 
THEOREM 7.1. Let the assumptions of Theorem 6.6 hold. Then there exists positive $\mu$ such that, for every two solutions $u_{1}, u_{2} \in v_{c}+\mathbb{W}_{b}([0, T] \times \Omega)$ and every weight function $\phi$ of sufficiently small exponential growth rate $\varepsilon(\varepsilon \leq \mu)$, the following estimate holds:

$$
\left\|u_{1}(t)-u_{2}(t)\right\|_{L_{\phi}^{2}(\Omega)} \leq C e^{K t}\left\|u_{1}(0)-u_{2}(0)\right\|_{L_{\phi}^{2}(\Omega)},
$$

where the constants $K$ and $C$ depend on the $L_{b}^{2}$-norms of $u_{1}(0)$ and $u_{2}(0), g$ and constant $C_{\phi}$, but are independent of the concrete choice of $u_{1}, u_{2}$ and $\phi$.

In particular, the weak energy solution of the Navier-Stokes is unique. Moreover, the analogous estimate holds for the spaces $L_{b, \phi}^{2}$ as well.

Proof. We first note that it is sufficient to verify (7.1) for $t \leq 1$ (for other $t$ it can then be obtained by iteration) and the weights $\varphi_{\mu, y}(s)$ only.

We introduce also a family of cut-off functions $\psi_{y}(s) \in[0,1], s \in \mathbb{R}$ such that

$$
\begin{aligned}
& \psi_{y}(s) \equiv 1, \quad s \in[y, y+1], \\
& \operatorname{supp} \psi_{y} \in(y-1, y+2), \\
& \left|D_{s}^{k} \psi_{y}(s)\right| \leq C_{k} .
\end{aligned}
$$

Let now $u_{1}, u_{2} \in v_{c}+\mathbb{W}_{b}([0, T] \times \Omega)$ be two solutions of the Navier-Stokes problem (6.1) and let $v:=u_{2}-u_{1} \in \mathbb{W}_{b}([0, T] \times \Omega)$. Then, this function satisfies

$\partial_{t} v+\Pi\left[\left(v, \nabla_{x}\right) v\right]+\Pi\left[\left(u_{1}, \nabla_{x}\right) v\right]+\Pi\left[\left(v, \nabla_{x}\right) u_{1}\right]=\Pi \Delta_{x} v, \operatorname{div} v=0,\left.v\right|_{\partial \Omega}=0$.

Let us now fix an arbitrary $t \in(0, T], y \in \mathbb{R}$ and construct the corrector function $v_{\psi}(\tau)=v_{\psi_{y}}^{t}(\tau)$ as the solution of the following analogue of (5.30):

$$
\begin{gathered}
-\partial_{\tau} v_{\psi}=\Delta_{x} v_{\psi}-\nabla_{x} q, \quad \operatorname{div} v_{\psi}(\tau)=2 \psi_{y} \psi_{y}^{\prime} v(\tau), \\
\left.\Pi v_{\psi}\right|_{\tau=t}=0,\left.\quad v_{\psi}\right|_{\partial \Omega}=0 .
\end{gathered}
$$

Then, applying Theorem 5.6 and (5.32) to equation (7.3), we have

$$
\begin{aligned}
1 / 2( & \left.\psi_{y}^{2} v(t), v(t)\right)+\int_{0}^{t}\left(\nabla_{x} v(\tau), \nabla_{x}\left(\psi_{y}^{2} v(\tau)\right)\right) d \tau \\
= & 1 / 2\left(\psi_{y}^{2} v(0), v(0)\right)-\left(v(0), v_{\psi}(0)\right)-\int_{0}^{t}\left(\left(u_{1}(\tau), \nabla_{x}\right) v(\tau)+\left(v(\tau), \nabla_{x}\right) v(\tau)\right. \\
& \left.\quad+\left(v(\tau), \nabla_{x}\right) u_{1}(\tau), \psi_{y}^{2} v(\tau)-v_{\psi}(\tau)\right) d \tau
\end{aligned}
$$

(here we have implicitly used that $\Pi v_{\psi}(t)=0$ ). We now recall that the function $h_{v, \psi}(\tau):=2 \psi_{y} \psi_{y}^{\prime} v(\tau)$ has the finite support with respect to $x_{1}$ (belonging to $(y-1$, $y+2)$ ). Consequently,

$$
\left\|h_{v, \psi}(\tau)\right\|_{W_{\varphi_{\varepsilon, y}^{l}}^{l, 2}(\Omega)} \leq C\|v(\tau)\|_{W_{\varphi_{\varepsilon, y}}^{l, 2}(\Omega)}, l=0,1
$$

where the constant $C$ is independent of $0<\varepsilon \leq 1$ and $y \in \mathbb{R}$. Moreover, since $v \in$ $\mathbb{W}_{b}([0, T] \times \Omega)$ the function $h_{v, \psi}$ also satisfies assumption (5.2). Thus, due to Theorem 5.1, we have the following estimates:

$$
\int_{0}^{t}\left\|v_{\psi}(\tau)\right\|_{W_{\varphi_{\varepsilon, y}^{-1}}^{2,2}(\Omega)}^{2} d \tau \leq C \int_{0}^{t}\|v(\tau)\|_{W_{\varphi, y}^{1,2}(\Omega)}^{2} d \tau
$$


and

$$
\left\|v_{\psi}(0)\right\|_{W_{\varphi_{\varepsilon, y}^{1,1}}^{1,2}(\Omega)}^{2} \leq C\|v(0)\|_{L_{\varphi_{\varepsilon, y}}^{2}(\Omega)}^{2}+C \int_{0}^{t}\|v(\tau)\|_{W_{\varphi_{\varepsilon, y},(\Omega)}^{1,2}}^{2} d \tau
$$

(where $\varepsilon \leq \mu>0$ is small enough and the constant $C$ is independent of $y \in \mathbb{R}$ ). Furthermore, due to Corollary 5.4, we also have

$$
\int_{0}^{t}\left\|v_{\psi}(\tau)\right\|_{L_{\varphi_{\varepsilon, y}^{2}}^{2}(\Omega)}^{2} d \tau \leq C \int_{0}^{t}\|v(\tau)\|_{L_{\varphi_{s, y}}^{2}(\Omega)}^{2} d \tau .
$$

Combining now estimates (7.9) and (7.7) and using the proper interpolation inequality, we deduce

$$
\begin{aligned}
& \int_{0}^{t}\left\|v_{\psi}(\tau)\right\|_{W_{\varphi_{\varepsilon, y}^{1}}^{1,2}(\Omega)}^{2}+\left\|v_{\psi}(\tau)\right\|_{L_{\varphi_{e, y}^{-1}}^{\infty}(\Omega)}^{2} d \tau \\
& \quad \leq C_{\delta} \int_{0}^{t}\|v(\tau)\|_{L_{\varphi_{\varepsilon, y}}^{2}(\Omega)}^{2} d \tau+\delta \int_{0}^{t}\|v(\tau)\|_{W_{\phi_{\varepsilon, y}}^{1,2}(\Omega)}^{2} d \tau
\end{aligned}
$$

where the constant $\delta>0$ is arbitrary and the constant $C_{\delta}$ is independent of $y \in \mathbb{R}$.

We are now ready to estimate the integral in the right-hand side of (7.5). We start with the terms containing the function $v_{\psi}(\tau)$. Indeed, since $u \in \mathbb{W}_{b}([0, T] \times \Omega)$ then, due to Theorem 5.6 and estimate (5.45), $\left\|u_{1}(t)\right\|_{L_{b}^{2}(\Omega)} \leq C$ and, consequently,

$\left|\left(\left(u_{1}, \nabla_{x}\right) v, v_{\psi}\right)\right| \leq C\left\|u_{1}\right\|_{L_{b}^{2}(\Omega)}\left\|\nabla_{x} v\right\|_{L_{\varphi_{\varepsilon, y}}^{2}(\Omega)}\left\|v_{\psi}\right\|_{L_{\varphi_{\varepsilon, y}^{-1}}^{\infty}(\Omega)} \leq \delta\|v\|_{W_{\varphi_{\varepsilon, y}}^{1,2}(\Omega)}^{2}+C_{\delta}\left\|v_{\psi}\right\|_{L_{\varphi_{\varepsilon, y}^{-1}}^{\infty}(\Omega)}^{2}$

which together with (7.10) gives

$$
\int_{0}^{t}\left|\left(\left(u_{1}(\tau), \nabla_{x}\right) v(\tau), v_{\psi}(\tau)\right)\right| d \tau \leq C_{\delta} \int_{0}^{t}\|v(\tau)\|_{L_{\varphi_{\varepsilon, y}}^{2},(\Omega)}^{2} d \tau+\delta \int_{0}^{t}\|v(\tau)\|_{W_{\varphi_{\varepsilon, y},}^{1,2}(\Omega)}^{2} d \tau
$$

where $\delta>0$ is arbitrary. Recalling now that $v=u_{2}-u_{1}$ and arguing analogously, we obtain also that

$$
\int_{0}^{t}\left|\left(\left(v(\tau), \nabla_{x}\right) v(\tau), v_{\psi}(\tau)\right)\right| d \tau \leq C_{\delta} \int_{0}^{t}\|v(\tau)\|_{L_{\psi_{\varepsilon, y}}^{2}(\Omega)}^{2} d \tau+\delta \int_{0}^{t}\|v(\tau)\|_{W_{\psi_{\varepsilon, y}}^{1,2}(\Omega)}^{2} d \tau .
$$

Moreover, integrating by parts, we have

$$
\left|\left(\left(v, \nabla_{x}\right) u_{1}, v_{\psi}\right)\right| \leq\left(\left|u_{1}\right|,\left|\nabla_{x} v\right| \cdot\left|v_{\psi}\right|\right)+\left(\left|u_{1}\right|,|v| \cdot\left|\nabla_{x} v_{\psi}\right|\right) .
$$

The first term in the right-hand side of this inequality can be estimated exactly as (7.11)-(7.12), so we only need to estimate the second one. Indeed, due to Proposition 2.14 and Hölder inequality,

$$
\begin{aligned}
& \left(\left|u_{1}\right|,|v| \cdot\left|\nabla_{x} v_{\psi}\right|\right) \leq C\left\|u_{1}\right\|_{L_{b}^{2}(\Omega)}\|v\|_{L_{\varphi_{\varepsilon, y}}^{4}(\Omega)}\left\|\nabla_{x} v_{\psi}\right\|_{L_{\varphi_{\varepsilon, y}^{-1}}^{4}(\Omega)} \\
& \leq C_{1}\left(\|v\|_{L_{\varphi_{\varepsilon}, y}^{2}(\Omega)}^{2}\|v\|_{W_{\varphi_{\varepsilon, y}}^{1,2}(\Omega)}\left\|v_{\psi}\right\|_{W_{\varphi_{\varepsilon, y}^{1, y}}^{1,2}(\Omega)}\left\|v_{\psi}\right\|_{W_{\varphi_{\varepsilon, y}^{-1}}^{2,2}(\Omega)}\right)^{1 / 2}
\end{aligned}
$$

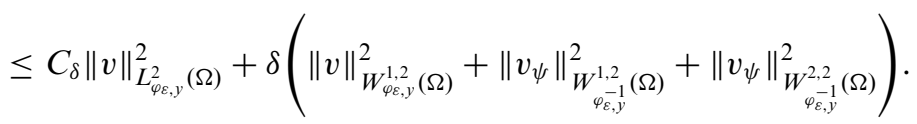


Using now estimate (7.7), we infer

$$
\int_{0}^{t}\left|\left(\left(v(\tau), \nabla_{x}\right) u(\tau), v_{\psi}(\tau)\right)\right| d \tau \leq C_{\delta} \int_{0}^{t}\|v(\tau)\|_{L_{\varphi, y}^{2}(\Omega)}^{2} d \tau+\delta \int_{0}^{t}\|v(\tau)\|_{W_{\varphi, y}^{1,2}(\Omega)}^{2} d \tau
$$

where the constant $\delta>0$ can be arbitrary. Thus, the terms under the integral in the right-hand side of (7.5) which contain the function $v_{\psi}$ are estimated. Let us now estimate the terms containing $\psi_{y}^{2} v$. Indeed, using the interpolation inequality of Proposition 2.14 and the fact that $\psi_{y}$ has a finite support, we get

$$
\begin{aligned}
\left|\left(\left(v, \nabla_{x}\right) u_{1}, \psi_{y}^{2} v\right)\right| & \leq C\left\|\nabla_{x} u_{1}\right\|_{L_{\varphi_{\varepsilon, y}}^{2}(\Omega)}\left\|\psi_{y} v\right\|_{L^{4}(\Omega)}^{2} \\
& \leq \beta\left\|\psi_{y} v\right\|_{W^{1,2}(\Omega)}^{2}+C_{\beta}\left\|u_{1}\right\|_{W_{\varphi_{\varepsilon, y}}^{1,2}(\Omega)}^{2}\left\|\psi_{y} v\right\|_{L^{2}(\Omega)}^{2}
\end{aligned}
$$

where the positive constant $\beta$ can be arbitrarily small and the constant $C_{\beta}$ is independent of $y \in \mathbb{R}$.

Moreover, integrating by parts and again using Proposition 2.14, we also deduce

$$
\begin{aligned}
\left|\left(\left(u_{1}, \nabla_{x}\right) v, \psi_{y}^{2} v\right)\right| & \leq 2 \mid\left(\left|u_{1}\right| \cdot|v|^{2},\left|\psi \psi^{\prime}\right|\right) \leq C\left\|u_{1}\right\|_{L_{b}^{2}(\Omega)}\|v\|_{L_{\varphi_{\varepsilon, y}}^{4}(\Omega)}^{2} \\
& \leq \delta\|v\|_{W_{\varphi, y}^{1,2}(\Omega)}^{2}+C_{\delta}\|v\|_{L_{\varphi_{\varepsilon, y}}^{2}(\Omega)}^{2}
\end{aligned}
$$

which gives

$$
\int_{0}^{t}\left|\left(\left(u_{1}(\tau), \nabla_{x}\right) v(\tau), \psi_{y}^{2} v(\tau)\right)\right| d \tau \leq \delta \int_{0}^{t}\|v(\tau)\|_{W_{\varphi_{\varepsilon, y}}^{1,2}(\Omega)}^{2} d \tau+C_{\delta} \int_{0}^{t}\|v(\tau)\|_{L_{\varphi_{\varepsilon, y}}^{2}(\Omega)}^{2} d \tau,
$$

where $\delta>0$ can be arbitrarily small. Recalling now that $v=u_{2}-u_{1}$ and arguing analogously, we prove that

$$
\int_{0}^{t}\left|\left(\left(v(\tau), \nabla_{x}\right) v(\tau), \psi_{y}^{2} v(\tau)\right)\right| d \tau \leq \delta \int_{0}^{t}\|v(\tau)\|_{W_{\varphi_{\varepsilon, y}}^{1,2}(\Omega)}^{2} d \tau+C_{\delta} \int_{0}^{t}\|v(\tau)\|_{L_{\varphi_{\varepsilon, y}}^{2}(\Omega)}^{2} d \tau .
$$

Thus, all of the integral terms in equality (7.5) are estimated. So, we only need to estimate two remaining terms. Indeed, due to estimate (7.8) and Hölder inequality, we have

$$
\begin{aligned}
\left|\left(v(0), v_{\psi}(0)\right)\right| & \leq C_{\delta}\|v(0)\|_{L_{\varphi_{\varepsilon, y}}^{2}(\Omega)}^{2}+\delta\left\|v_{\psi}(0)\right\|_{L_{\varphi_{\varepsilon, y}^{-1}}^{2}(\Omega)}^{2} \\
& \leq C_{\delta}\|v(0)\|_{L_{\varphi_{\varepsilon, y}}^{2}(\Omega)}^{2}+\delta \int_{0}^{t}\|v(\tau)\|_{W_{\varphi_{\varepsilon, y}}^{1,2}(\Omega)}^{2} d \tau
\end{aligned}
$$

where $\delta>0$ is again arbitrary.

Finally, it is not difficult to prove, integrating by parts, that

$$
\left(\nabla_{x} v, \nabla_{x}\left(\psi_{y}^{2} v\right)\right) \geq 1 / 2\left(\left\|\psi_{y} \nabla_{x} v\right\|_{L^{2}(\Omega)}^{2}+\left\|\nabla_{x}\left(\psi_{y} v\right)\right\|_{L^{2}(\Omega)}^{2}\right)-C\|v\|_{L_{\varphi_{\varepsilon}, y}^{2}(\Omega)}^{2} .
$$


Inserting estimates (6.10) and (7.12)-(7.19) to equality (7.5) and fixing the arbitrary positive constant $\beta$ (involved in (7.15)) to be small enough, we deduce that

$$
\begin{aligned}
\left\|\psi_{y} v(t)\right\|_{L^{2}(\Omega)}^{2} & +\int_{0}^{t}\left\|\psi_{y} \nabla_{x} v(\tau)\right\|_{L^{2}(\Omega)}^{2} d \tau \leq C \int_{0}^{t}\left\|\nabla_{x} u_{1}(\tau)\right\|_{L_{\varphi_{\varepsilon, y}}^{2}(\Omega)}^{2}\left\|\psi_{y} v(\tau)\right\|_{L^{2}(\Omega)}^{2} d \tau \\
& +\delta \int_{0}^{t}\|v(\tau)\|_{W_{\varphi_{\varepsilon, y}}^{1,2}(\Omega)}^{2} d \tau+C_{\delta} \int_{0}^{t}\|v(\tau)\|_{L_{\varphi_{\varepsilon, y}}^{2}(\Omega)}^{2} d \tau+C_{\delta}\|v(0)\|_{L_{\varphi_{\varepsilon, y}}^{2}(\Omega)}^{2}
\end{aligned}
$$

where the positive constant $\delta$ can be arbitrarily small and the constants $C$ and $C_{\delta}$ are independent of $y \in \mathbb{R}$ (it is very important that the constant $C$ in (7.20) is independent also of $\delta$ ). In order to transform (7.20), we need the following standard version of the Gronwall inequality.

LEMMA 7.2. Let the function $Z \in C([0,1])$ satisfy the following integral inequality:

$$
Z(t) \leq \int_{0}^{t} H(\tau) Z(\tau)+P(\tau) d t+K, \quad t \in[0,1]
$$

for some integrable functions $H, P \in L^{1}([0,1])$ such that $H(t) \geq 0$ and some constant $K$. Then, the following estimate holds:

$$
Z(t) \leq C|K|+C \int_{0}^{t}|P(t)| d t, \quad t \in[0,1]
$$

where the constant $C$ depends only on $\|H\|_{L^{1}([0,1])}$.

Proof. Indeed, let $W(t):=\int_{0}^{t}(H(\tau) Z(\tau)+P(\tau)) d \tau$. Then, due to (7.21), this function satisfies the following differential inequality:

$$
W^{\prime}(t) \leq H(t) W(t)+K H(t)+P(t), \quad t \in[0,1] .
$$

Integrating this inequality and using $W(0)=0$, we get

$$
\begin{aligned}
W(t) & \leq \int_{0}^{t} e^{\int_{s}^{t} H(\tau) d \tau}(P(s)+K H(s)) d s \\
& \leq e^{\|H\|_{L^{1}([0,1])}}\left(\int_{0}^{t}|P(\tau)| d \tau+|K| \cdot\|H\|_{L^{1}([0,1])}\right) .
\end{aligned}
$$

Inserting this estimate in the right-hand side of (7.21), we deduce (7.22) and finish the proof of the lemma.

We are now able to finish the proof of the theorem. To this end, we apply Lemma 7.2 to inequality (7.20) with $Z(t):=\left\|\psi_{y} v(t)\right\|_{L^{2}(\Omega)}^{2}$. Then, using the fact that the integrals $\int_{0}^{1}\left\|u_{1}(\tau)\right\|_{W_{\varphi, y}^{1,2}(\Omega)}^{2} d \tau$ are uniformly (with respect to $y \in \mathbb{R}$ ) bounded (since $u_{1} \in v_{c}+$ $\left.\mathbb{W}_{b}([0, T] \times \Omega)\right)$, we have

$$
\begin{aligned}
\left\|\psi_{y} v(t)\right\|_{L^{2}(\Omega)}^{2} & +\int_{0}^{t}\left\|\psi_{y} \nabla_{x} v(\tau)\right\|_{L^{2}(\Omega)}^{2} d \tau \leq C_{\delta}\|v(0)\|_{L_{\varphi_{\varepsilon, y}}^{2}(\Omega)}^{2} \\
& +\delta \int_{0}^{t}\|v(\tau)\|_{W_{\varphi_{\varepsilon, y}}^{1,2}(\Omega)}^{2} d \tau+C_{\delta} \int_{0}^{t}\|v(\tau)\|_{L_{\varphi_{\varepsilon, y}}^{2}(\Omega)}^{2} d \tau, \quad t \in[0,1],
\end{aligned}
$$


where the constant $\delta>0$ can be arbitrarily small and $C_{\delta}$ is independent of $y \in \mathbb{R}$. Recalling now that $\psi_{y} \geq 0$ and equals 1 identically if $s \in[y, y+1]$, we transform (7.23) as follows:

$$
\begin{aligned}
\|v(t)\|_{L^{2}\left(\Omega_{y}\right)}^{2} & +\int_{0}^{t}\left\|\nabla_{x} v(\tau)\right\|_{L^{2}\left(\Omega_{y}\right)}^{2} d \tau \leq C_{\delta}\|v(0)\|_{L_{\varphi_{\varepsilon, y}}^{2}(\Omega)}^{2} \\
& +\delta \int_{0}^{t}\|v(\tau)\|_{W_{\varphi_{\varepsilon, y}}^{1,2}(\Omega)}^{2} d \tau+C_{\delta} \int_{0}^{t}\|v(\tau)\|_{L_{\varphi_{\varepsilon, y}}^{2}(\Omega)}^{2} d \tau, \quad t \in[0,1] .
\end{aligned}
$$

Multiplying this inequality by $\varphi_{\gamma, z}(y)$ with $\gamma<\varepsilon$, integrating over $y \in \mathbb{R}$ and using (2.12) with $q=1$, we deduce that

$$
\begin{aligned}
\|v(t)\|_{L_{\varphi_{\gamma, z}}^{2}(\Omega)}^{2} & +\int_{0}^{t}\|v(\tau)\|_{W_{\varphi_{\gamma, z}}^{1,2}(\Omega)}^{2} d \tau \leq C_{\delta}\|v(0)\|_{L_{\varphi_{\gamma, z}}^{2}(\Omega)}^{2} \\
& +\delta \int_{0}^{t}\|v(\tau)\|_{W_{\varphi_{Y, z}}^{1,2}(\Omega)}^{2} d \tau+C_{\delta} \int_{0}^{t}\|v(\tau)\|_{L_{\varphi_{\gamma, z}}^{2}(\Omega)}^{2} d \tau, \quad t \in[0,1],
\end{aligned}
$$

where $\delta>0$ is still arbitrary and $C_{\delta}$ is independent of $z \in \mathbb{R}$. Fixing $\delta$ to be small enough (say, $\delta=1 / 2$ ), we finally arrive at

$$
\begin{aligned}
\|v(t)\|_{L_{\varphi_{\gamma, z}}^{2}(\Omega)}^{2} & +\int_{0}^{t}\|v(\tau)\|_{W_{\varphi_{\gamma, z}}^{1,2}(\Omega)}^{2} d \tau \\
& \leq C\|v(0)\|_{L_{\varphi_{\gamma, z}}^{2}(\Omega)}^{2}+C \int_{0}^{t}\|v(\tau)\|_{L_{\varphi_{\gamma, z}}^{2}(\Omega)}^{2} d \tau, \quad t \in[0,1] .
\end{aligned}
$$

Applying now the Gronwall's inequality to this relation, we obtain

$$
\|v(t)\|_{L_{\varphi_{Y, z}}^{2}(\Omega)}^{2}+\int_{0}^{t}\|v(\tau)\|_{W_{\varphi_{\gamma, z}}^{1,2}(\Omega)}^{2} d \tau \leq C\|v(0)\|_{L_{\varphi_{\gamma, z}}^{2}(\Omega)}^{2}, \quad t \in[0,1] .
$$

Estimate (7.1) is an immediate corollary of (7.25) and (2.12). Theorem 7.1 is proved.

REMARK 7.3. In the proof of Theorem 7.1, it seemed natural to multiply equation (7.3) by $\varphi_{\varepsilon, y}^{2} v(t)-v_{\varphi_{\varepsilon, y}}(t)$ where $\varphi_{\varepsilon, y}$ is defined by (2.4) (instead of using the cut-off functions $\psi_{y}$ ). However, this scheme works only if the estimate

$$
\int_{0}^{t}\left\|\nabla_{x} u_{1}(\tau)\right\|_{L_{b}^{2}(\Omega)}^{2} d \tau=\int_{0}^{t} \sup _{y \in \mathbb{R}}\left\|\nabla_{x} u_{1}(\tau)\right\|_{L^{2}\left(\Omega_{y}\right)}^{2} d \tau \leq C_{t}
$$

is a priori known, but we have only that $\nabla_{x} u_{1} \in L_{b}^{2}\left([0, T], L_{b}^{2}(\Omega)\right)$. This means

$$
\sup _{y \in \mathbb{R}} \int_{0}^{t}\left\|\nabla_{x} u_{1}(\tau)\right\|_{L^{2}\left(\Omega_{y}\right)}^{2} d \tau \leq C_{t}
$$

see Remark 2.4. It is worth emphasizing that (7.27) is weaker than (7.26) and, in fact, we do not know how to control the integral (7.26) of a weak solution $u_{1}(t)$. In order to overcome this difficulty, we use (in the proof of Theorem 7.1) the localization technique based on the cut-off functions $\psi_{y}$. This technique allows us to prove the uniqueness using the weaker inequality (7.27) which follows from the assumption $u_{1} \in v_{c}+\mathbb{W}_{b}([0, T] \times \Omega)$. 
Our next task is to verify that the weak solution $u(t)$ of the Navier-Stokes problem (6.1) becomes more regular for $t>0$. To be more precise, the following smoothing property holds.

THEOREM 7.4. Let the assumptions of Theorem 6.6 be satisfied and let $u \in v_{c}+$ $\mathbb{W}_{b}([0, T] \times \Omega)$ be a weak solution of $(6.1)$ constructed in that theorem. Then,

$$
t^{1 / 2} u(t) \in L^{\infty}\left([0, T], W_{b}^{1,2}(\Omega)\right) \cap L_{b}^{2}\left([0, T], W_{b}^{2,2}(\Omega)\right)
$$

and the following estimate holds:

$$
t\|v(t)\|_{W^{1,2}\left(\Omega_{y}\right)}^{2}+\int_{0}^{t} \tau\|u(\tau)\|_{W^{2,2}\left(\Omega_{y}\right)}^{2} d \tau \leq Q\left(\left\|u_{0}\right\|_{L_{b}^{2}(\Omega)}+\|g\|_{L_{b}^{2}([0, T] \times \Omega)}\right), \quad t \in[0,1]
$$

where the monotonic function $Q$ is independent of $y \in \mathbb{R}$.

Proof. We give below only the formal derivation of estimate (7.29) which can be easily justified using the approximations of a solution $u$ by the square integrable solutions as in the proof of Theorem 6.5 (we recall that the uniqueness theorem is already proven and, consequently, every solution can be obtained by this procedure). Moreover, for simplicity, we restrict ourselves to the case of zero flux $\mathbb{S} u_{1} \equiv 0$ only. The general case $\mathbb{S} u_{1}=c$ can be easily reduced to that one by introducing the new dependent variable $v(t):=u(t)-v_{c}$ where $v_{c}=v_{c}\left(x_{2}\right)$ is a classical Poiseuille flow (see (6.47) and the proof of Theorem 6.6).

Now let $y \in \mathbb{R}$ be arbitrary and the cut-off functions $\psi_{y}$ satisfy (7.2). Let us multiply equation (6.9) by

$$
t\left(\partial_{x_{1}}\left(\psi_{y}^{2} \partial_{x_{1}} u\right)+\psi_{y}^{2} \partial_{x_{2}}^{2} u\right)=t \psi_{y}^{2} \Delta_{x} u+2 t \psi_{y} \psi_{y}^{\prime} \partial_{x_{1}} u
$$

and integrate over $[0, T] \times \Omega$. Then we have

$$
\begin{aligned}
& T\left\|\psi_{y} \nabla_{x} u(T)\right\|_{L^{2}(\Omega)}^{2}+2 \int_{0}^{T} t\left(\Pi \Delta_{x} u(t), \psi_{y}^{2} \Delta_{x} u(t)\right) d t \\
& =\int_{0}^{T}\left\|\psi_{y} \nabla_{x} u(t)\right\|_{L^{2}(\Omega)}^{2} d t-4 \int_{0}^{T} t\left(\Pi \Delta_{x} u, \psi_{y} \psi_{y}^{\prime} \partial_{x_{1}} u\right) d t \\
& \quad+2 \int_{0}^{T} t\left(\Pi\left[\left(u, \nabla_{x}\right) u\right], \psi_{y}^{2} \Delta_{x} u\right) d t+4 \int_{0}^{T} t\left(\Pi\left[\left(u, \nabla_{x}\right) u\right], \psi_{y} \psi_{y}^{\prime} \partial_{x_{1}} u\right) d t \\
& \quad-2 \int_{0}^{T} t\left(\Pi g, \psi_{y}^{2} \Delta_{x} u+2 \psi_{y} \psi_{y}^{\prime} \partial_{x_{1}} u\right) d t
\end{aligned}
$$

In order to estimate different terms in equality (7.31), we need the following lemma which gives the analogue of (4.17) and (4.25) for the cut-off functions $\psi_{y}$.

LEMMA 7.5. There exists a sufficiently small $\varepsilon>0$ such that, for every $u \in \mathcal{V}_{b}(\Omega) \cap$ $\mathcal{H}_{b}^{2,2}(\Omega)$ and every $y \in \mathbb{R}$ the following estimate holds:

$$
\left\|\psi_{y} u\right\|_{W^{2,2}(\Omega)}^{2} \leq C\left\|\psi_{y} \Pi \Delta_{x} u\right\|_{L^{2}(\Omega)}^{2}+\delta\|u\|_{W_{\varphi, y}^{2,2}(\Omega)}^{2}+C_{\delta}\|u\|_{L_{\varphi_{\varepsilon, y}}^{2}(\Omega)}^{2}
$$

where $\delta>0$ can be arbitrary, the constants $C$ and $C_{\delta}$ are independent of $y$ and $u$ and, in addition, the constant $C$ is independent also of $\delta$. 
Moreover, for every $u \in\left[L_{b}^{2}(\Omega)\right]^{2}$, the following estimate holds:

$$
\left\|\left(\psi_{y} \circ \Pi-\Pi \circ \psi_{y}\right) u\right\|_{W_{\substack{1,-1 \\ \phi_{\varepsilon, y}}}^{1,2}(\Omega)} \leq C\|u\|_{L_{\varphi_{\varepsilon, y}}^{2}(\Omega)}
$$

where the constant $C$ is also independent of $y$ and $u$.

Proof. Indeed, let $h:=\Pi \Delta_{x} u$. Then, the function $u$ solves the following Stokes problem:

$$
\Delta_{x} u+\nabla_{x} q=h, \quad \operatorname{div} u=0,\left.\quad u\right|_{\partial \Omega}=0 .
$$

Introducing the stream function $\Phi$ associated with the divergent free vector field $u$ (see (4.3) and (4.4)), we rewrite this equation as follows:

$$
\Delta_{x}^{2} \Phi=\partial_{x_{2}} h_{1}-\partial_{x_{1}} h_{2},\left.\quad \Phi\right|_{\partial \Omega}=\left.\nabla_{x} \Phi\right|_{\partial \Omega}=0
$$

which, in turns, implies that

$$
\Delta_{x}^{2}\left(\psi_{y} \Phi\right)=\partial_{x_{2}}\left(\psi_{y} h_{1}\right)-\partial_{x_{1}}\left(\psi_{y} h_{2}\right)+\psi_{y}^{\prime} h_{2}+T\left(\Phi, \psi_{y}\right)
$$

where the operator $T$ contains the derivatives of $\Phi$ of order at most 3 and, consequently, satisfies

$$
\|T\|_{W^{-1,2}(\Omega)} \leq C\|\Phi\|_{W_{\varphi,, y}^{2,2}(\Omega)} .
$$

Moreover, according to Propositions 3.3 and 4.11, we have

$$
\|\Phi\|_{W_{\varphi_{\varepsilon, y}^{2}, 2}^{2,2}(\Omega)} \leq C\left\|\Pi \Delta_{x} u\right\|_{\mathcal{H}_{\phi_{\varepsilon, y}}^{-1,2}(\Omega)} \leq C_{1}\|u\|_{\mathcal{H}_{\varphi_{\varepsilon, y}}^{1}(\Omega)} .
$$

Using now Proposition 3.3 once more, we deduce from (7.36)-(7.38) that

$$
\left\|\psi_{y} \Phi\right\|_{W^{3,2}(\Omega)} \leq C\left(\left\|\psi_{y} \Pi \Delta_{x} u\right\|_{L_{\varphi_{\varepsilon, y}}^{2}(\Omega)}+\|u\|_{\mathcal{V}_{\varphi_{\varepsilon, y}}(\Omega)}\right)+C\left\|\psi_{y}^{\prime} h_{2}\right\|_{W^{-1,2}(\Omega)} .
$$

So, we need only estimate the $W^{-1,2}$-norm of $\psi_{y}^{\prime} h_{2}=\psi_{y}^{\prime}\left(\Pi \Delta_{x} u\right)_{2}$. Indeed, let $\phi \in$ $W_{0}^{1,2}(\Omega)$ be arbitrary and let $\tilde{\phi} \in \mathcal{H}^{1,2}(\Omega)$ be computed via

$$
\tilde{\phi}:=\Pi\left(\begin{array}{c}
0 \\
\psi_{y}^{\prime} \phi
\end{array}\right) .
$$

Then, according to Theorem 4.4, we have

$$
\|\tilde{\phi}\|_{\mathcal{H}_{\varphi_{\varepsilon, y}^{1}, 2}^{1,2}(\Omega)} \leq C\|\phi\|_{W^{1,2}(\Omega)} .
$$

On the other hand, integrating by parts, we arrive at

$$
\left(\psi_{y}^{\prime}\left(\Pi \Delta_{x} u\right)_{2}, \phi\right)=\left(\Delta_{x} u, \tilde{\phi}\right)=-\left(\nabla_{x} u, \nabla_{x} \tilde{\phi}\right)+\left(\partial_{n} u, \tilde{\phi}\right)_{\partial \Omega} .
$$

Thus, due to the trace part of Proposition 2.10, we have

$$
\begin{aligned}
& \left|\left(\psi_{y}^{\prime} h, \phi\right)\right| \leq\|u\|_{W_{\varphi_{\varepsilon, y}}^{1,2}(\Omega)}\|\tilde{\phi}\|_{W_{\varphi_{\varepsilon, y}^{1,}}^{1,2}(\Omega)} \\
& \quad+\left\|\partial_{n} u\right\|_{L_{\varphi_{\varepsilon, y}}^{2}(\partial \Omega)}\|\tilde{\phi}\|_{L_{\varphi_{\varepsilon, y}^{-1}}^{2}(\partial \Omega)} \leq C\|u\|_{W_{\varphi_{\varepsilon, y}}^{7 /, 2}(\Omega)}\|\phi\|_{W^{1,2}(\Omega)} .
\end{aligned}
$$


Inserting estimate (7.42) into the right-hand side of (7.39) and using the standard interpolation inequality

$$
\|u\|_{W_{\varphi_{\varepsilon, y}}^{7 / 4,2}(\Omega)} \leq \delta\|u\|_{W_{\varphi_{\varepsilon, y}}^{2,2}(\Omega)}+C_{\delta}\|u\|_{L_{\varphi_{\varepsilon, y}}^{2}(\Omega)}
$$

we obtain

$$
\left\|\psi_{y} \Phi\right\|_{W^{3,2}(\Omega)} \leq C\left\|\psi_{y} \Delta_{x} u\right\|_{L^{2}(\Omega)}+\delta\|u\|_{W_{\varphi,, y}^{2,2}(\Omega)}+C_{\delta}\|u\|_{L_{\varphi_{\varepsilon, y}}^{2}(\Omega)} .
$$

Since $u_{1}=\partial_{x_{2}} \Phi$ and $u_{2}=-\partial_{x_{1}} \Phi$, estimate (7.44) (together with (7.38)) implies inequality (7.32). Thus, the first part of the lemma is proved.

Let us now prove the second part (inequality (7.33)). Indeed, let $\Phi_{u}$ and $\Phi_{y, u}$ be the stream functions associated with the divergent free vector fields $\Pi u$ and $\Pi\left(\psi_{y} u\right)$ respectively. Then, analogously to (4.18), the difference $W:=\psi_{y} \Phi_{u}-\Phi_{y, u}$ satisfies the following equation:

$$
\Delta_{x} W=H:=2 \psi_{y}^{\prime} \partial_{x_{1}} \Phi_{u}+\psi_{y}^{\prime \prime} \Phi_{u}+\psi_{y}^{\prime} u_{2},\left.\quad W\right|_{\partial \Omega}=0 .
$$

Thus, due to Proposition 3.1, we have

$$
\|W\|_{W_{\varphi_{\varepsilon, y}^{-1}}^{2,2}(\Omega)} \leq C\|H\|_{L_{\varphi_{\varepsilon, y}^{-1}}^{2}(\Omega)} \leq C_{1}\left\|\Phi_{u}\right\|_{W_{\varphi_{\varepsilon, y}}^{1,2}(\Omega)}+C_{1}\|u\|_{L_{\varphi_{\varepsilon, y}}^{2}(\Omega)} \leq C_{2}\|u\|_{L_{\varphi_{\varepsilon, y}}^{2}(\Omega)}
$$

which implies estimate (7.33) (see the end of the proof of Proposition 4.7). Thus, Lemma 7.5 is proved.

We are now ready to estimate the integrals involved in identity (7.31). We start with the integral in the left-hand side. Indeed, denoting $L u:=\left(\psi_{y}^{2} \Pi-\Pi \psi_{y}^{2}\right) \Delta_{x} u$ and integrating by parts, we get

$$
\begin{aligned}
\left(\Pi \Delta_{x} u, \psi_{y}^{2} \Delta_{x} u\right) & =\left\|\psi_{y} \Pi \Delta_{x} u\right\|_{L^{2}(\Omega)}^{2}-\left(\Delta_{x} u, \Pi L u\right) \\
& =\left\|\psi_{y} \Pi \Delta_{x} u\right\|_{L^{2}(\Omega)}^{2}+\left(\nabla_{x} u, \nabla_{x} \Pi L u\right)-\left(\partial_{n} u, \Pi L u\right)_{\partial \Omega} .
\end{aligned}
$$

Moreover, due to Lemma 7.5, estimate (7.33) and Theorem 4.4, we also obtain

$$
\|\Pi L u\|_{W_{\varphi_{\varepsilon, y}^{1,2}}^{1,2}(\Omega)}+\|L u\|_{W_{\varphi_{\varepsilon, y}^{-1}}^{1,2}(\Omega)} \leq C\left\|\Delta_{x} u\right\|_{L_{\varphi_{\varepsilon, y}}^{2}(\Omega)} .
$$

Now using (6.47) and arguing exactly as (7.41)-(7.43), we obtain

$$
\left|\left(\nabla_{x} u, \nabla_{x} \Pi L u\right)\right|+\left|\left(\partial_{n} u, \Pi L u\right)_{\partial \Omega}\right| \leq \delta\|u\|_{W_{\varphi_{\varepsilon}, y}^{2,2}(\Omega)}^{2}+C_{\delta}\|u\|_{L_{\varphi_{\varepsilon, y}}^{2}(\Omega)}^{2}
$$

where the constant $C_{\delta}$ is independent of $y$ and $u$ and the constant $\delta>0$ can be chosen arbitrarily small. Inserting estimates (7.48) and (7.32) into the right-hand side of (7.46), we arrive at

$$
\begin{aligned}
\left(\Pi \Delta_{x} u, \psi_{y}^{2} \Delta_{x} u\right) \geq & \alpha\left(\left\|\psi_{y} \Pi \Delta_{x} u\right\|_{L^{2}(\Omega)}^{2}+\left\|\psi_{y} u\right\|_{W^{2,2}(\Omega)}^{2}\right) \\
& -\delta\|u\|_{W_{\varphi_{\varepsilon, y}}^{2,2}(\Omega)}^{2}-C_{\delta}\|u\|_{L_{\varphi_{\varepsilon, y}}^{2}(\Omega)}^{2}
\end{aligned}
$$

where $\alpha>0$ is some fixed constant which is independent of $y, u$ and $\delta$ and $\delta>0$ can be chosen arbitrarily. Recalling now that $u(t)$ is a solution of the Navier-Stokes problem 
(6.1) which is bounded in $\mathbb{W}_{b}([0, T] \times \Omega)$, we finally deduce that

$$
\begin{aligned}
& \int_{0}^{T} t\left(\Pi \Delta_{x} u(t), \psi_{y}^{2} \Delta_{x} u(t)\right) d t \\
& \quad \geq \alpha \int_{0}^{T} t\left(\left\|\psi_{y} \Pi \Delta_{x} u(t)\right\|_{L^{2}(\Omega)}^{2}+\left\|\psi_{y} u(t)\right\|_{W^{2,2}(\Omega)}^{2}\right) d t-\delta \int_{0}^{T} t\|u(t)\|_{W_{\varphi_{\varepsilon, y}^{2,}(\Omega)}^{2}}^{2} d t-C_{\delta}
\end{aligned}
$$

where $T \in[0,1]$, the constant $C_{\delta}$ depends on $\delta$ and on the $\mathbb{W}_{b}$-norm of the solution $u$, and the constant $\alpha>0$ is independent of $\delta$ and $u$.

Let us now estimate the most complicated third integral in the right-hand side of (7.31). To this end, we transform it as follows:

$$
\begin{aligned}
& \left(\Pi\left[\left(u, \nabla_{x}\right) u\right], \psi_{y}^{2} \Delta_{x} u\right. \\
& \quad=\left(\left(u, \nabla_{x}\right)\left(\psi_{y} u\right), \psi_{y} \Pi \Delta_{x} u\right)-\left(\left(u, \nabla_{x}\right) u, L u\right)-\left(u_{1} \psi_{y}^{\prime} u, \psi_{y} \Pi \Delta_{x} u\right) .
\end{aligned}
$$

where $L u$ is the same as in (7.46). Using estimate (7.47), embedding $W^{1,2} \subset L^{4}$ and the interpolation inequality (2.39), we have

$$
\begin{aligned}
\left|\left(\left(u, \nabla_{x}\right) u, L u\right)\right| & \leq C\|u\|_{L_{b}^{2}(\Omega)}\left\|\nabla_{x} u\right\|_{L_{\varphi_{\varepsilon, y}^{4}}^{4}(\Omega)}\|L u\|_{L_{\varphi_{\varepsilon, y}^{4}}^{4}(\Omega)} \\
& \leq C_{1}\|u\|_{L_{b}^{2}(\Omega)}\|u\|_{W_{\varphi_{\varepsilon, y}}^{1,2}(\Omega)}^{1 / 2}\|u\|_{W_{\varphi_{\varepsilon, y}}^{2,2}(\Omega)}^{3 / 2} \\
& \leq C_{\delta}\|u\|_{L_{b}^{2}(\Omega)}^{4}\|u\|_{W_{\varphi_{\varepsilon, y}}^{1,2}(\Omega)}^{2}+\delta\|u\|_{W_{\varphi_{\varepsilon, y}}^{2,2}(\Omega)}^{2}
\end{aligned}
$$

Multiplying this inequality by $t$, integrating over $[0, T], T \in[0,1]$, and using that the solution $u \in \mathbb{W}_{b}([0, T] \times \Omega)$, we deduce that

$$
\left.\int_{0}^{T} t \mid\left(u(t), \nabla_{x}\right) u(t), L u(t)\right) \mid d t \leq C_{\delta}+\delta \int_{0} t\|u(t)\|_{W_{\varphi_{\varepsilon, y}}^{2,2}(\Omega)}^{2} d t
$$

where $\delta>0$ is arbitrary and $C_{\delta}$ depends on the $\mathbb{W}_{b}$-norm of $u$. Moreover, arguing analogously, we can estimate the integral from the third term in the right-hand side of (7.51) as follows:

$$
\int_{0}^{T}\left|\left(u_{1} \psi_{y}^{\prime} u, \psi_{y} \Pi \Delta_{x} u\right)\right| d t \leq C_{\delta}+\delta \int_{0} t\|u(t)\|_{W_{\varphi_{\varepsilon, y}}^{2,2}(\Omega)}^{2} d t .
$$

We are now ready to estimate the first term in the right-hand side of (7.51). Indeed, using again the interpolation inequality (2.39) and the fact that $\psi_{y}$ has a finite support, we deduce

$$
\begin{aligned}
& \left|\left(\left(u, \nabla_{x}\right)\left(\psi_{y} u\right), \psi_{y} \Pi \Delta_{x} u\right)\right| \leq C\left\||u| \cdot\left|\nabla_{x}\left(\psi_{y} u\right)\right|\right\|_{L^{2}(\Omega)}^{2}+\alpha / 4\left\|\psi_{y} \Pi \Delta_{x} u\right\|_{L^{2}(\Omega)}^{2} \\
& \quad \leq C\|u\|_{L_{\varphi_{\ell}, y}^{4}(\Omega)}^{2}\left\|\nabla_{x}\left(\psi_{y} u\right)\right\|_{L^{4}(\Omega)}^{2}+\alpha / 4\left\|\psi_{y} \Pi \Delta_{x} u\right\|_{L^{2}(\Omega)}^{2} \\
& \quad \leq C_{1}\|u\|_{L_{\varphi_{\varepsilon, y}}^{2}(\Omega)}\|u\|_{W_{\varphi_{\varepsilon, y}}^{1,2}(\Omega)}\left\|\nabla_{x}\left(\psi_{y} u\right)\right\|_{L^{2}(\Omega)}\left\|\psi_{y} u\right\|_{W^{2,2}(\Omega)}+\alpha / 4\left\|\psi_{y} \Pi \Delta_{x} u\right\|_{L^{2}(\Omega)}^{2} \\
& \quad \leq C_{2}\|u\|_{L_{\varphi_{\varepsilon, y}}^{2}(\Omega)}^{2}\|u\|_{W_{\varphi_{,}, y}^{1,2}(\Omega)}^{2}\left\|\nabla_{x}\left(\psi_{y} u\right)\right\|_{L^{2}(\Omega)}^{2}+\alpha / 4\left(\left\|\psi_{y} \Pi \Delta_{x} u\right\|_{L^{2}(\Omega)}^{2}+\left\|\psi_{y} u\right\|_{W^{2,2}(\Omega)}^{2}\right)
\end{aligned}
$$


where the constant $\alpha$ is the same as in (7.50). Estimates (7.51)-(7.54) together with the fact that $u$ belongs to $\mathbb{W}_{b}([0, T] \times \Omega)$ give the following estimate:

$$
\begin{aligned}
& \left.\int_{0}^{T} t \mid\left(\Pi\left[u(t), \nabla_{x}\right) u(t)\right], \psi_{y}^{2} \Delta_{x} u(t)\right) \mid d t \leq C_{\delta}+C \int_{0}^{T} t\|u(t)\|_{W_{\varphi_{\varepsilon, y}}^{1,2}(\Omega)}^{2}\left\|\nabla_{x}\left(\psi_{y} u\right)\right\|_{L^{2}(\Omega)}^{2} d t \\
& \quad+\alpha / 4 \int_{0}^{T} t\left(\left\|\psi_{y} \Pi \Delta_{x} u\right\|_{L^{2}(\Omega)}^{2}+\left\|\psi_{y} u\right\|_{W^{2,2}(\Omega)}^{2}\right) d t+\delta \int_{0}^{T} t\|u(t)\|_{W_{\varphi, y}^{2,2}(\Omega)}^{2} d t .
\end{aligned}
$$

Thus, the most complicated third term in the right-hand side of (7.31) is estimated. The remaining terms are much simpler. Indeed, arguing analogously to estimating the second term in the right-hand side of (7.51), we have

$$
\int_{0}^{T} t\left|\left(\Pi\left[\left(u(t), \nabla_{x}\right) u(t)\right], \psi_{y} \psi_{y}^{\prime} \partial_{x_{1}} u(t)\right)\right| d t \leq C_{\delta}+\delta \int_{0}^{T} t\|u(t)\|_{W_{\varphi_{\varepsilon, y}}^{2,2}(\Omega)}^{2} d t .
$$

The term containing $\Pi g$ can be easily estimated by Hölder inequality:

$$
\begin{aligned}
& \int_{0}^{T} t\left|\left(\Pi g(t), \psi_{y}^{2} \Delta_{x} u(t)+2 \psi_{y} \psi_{y}^{\prime} \partial_{x_{1}} u(t)\right)\right| d t \leq C \int_{0}^{T} t\|g(t)\|_{L_{\psi_{\varepsilon, y}}^{2}(\Omega)}\|u\|_{W_{\varphi_{\varepsilon, y}}^{2,2}(\Omega)} d t \\
& \quad \leq C_{\delta}\|g\|_{L_{b}^{2}([0, T] \times \Omega)}^{2}+\delta \int_{0}^{T} t\|u\|_{W_{\varphi_{\varepsilon, y}}^{2,2}(\Omega)}^{2} d t .
\end{aligned}
$$

Analogously,

$$
\int_{0}^{T} t\left|\left(\Pi \Delta_{x} u(t), \psi_{y} \psi_{y}^{\prime} \partial_{x_{1}} u(t)\right)\right| d t \leq C_{\delta}+\delta \int_{0}^{T} t\|u(t)\|_{W_{\varphi_{\varepsilon, y}}^{2,2}(\Omega)}^{2} d t .
$$

And, finally, the first term in the right-hand side of (7.31) is obviously bounded since $u \in \mathbb{W}_{b}([0, T] \times \Omega)$.

Inserting estimates (7.50), (7.55) and (7.56)-(7.58) into identity (7.31), we deduce the following integral inequality

$$
\begin{aligned}
& T\left\|\nabla_{x}\left(\psi_{y} u(T)\right)\right\|_{L^{2}(\Omega)}^{2}+\alpha \int_{0}^{T} t\left\|\psi_{y} u(t)\right\|_{W_{\varphi_{\varepsilon, y}}^{2,2}(\Omega)}^{2} d t \\
& \quad \leq C \int_{0}^{T}\|u(t)\|_{W_{\varphi_{\varepsilon}, y}^{1,2}(\Omega)}^{2}\left(t\left\|\nabla_{x}\left(\psi_{y} u(t)\right)\right\|_{L^{2}(\Omega)}^{2}\right) d t+C_{\delta}+\delta \int_{0}^{T} t\|u(t)\|_{W_{\varphi_{\varepsilon}, y}^{2,2}(\Omega)}^{2} d t
\end{aligned}
$$

where $T \in[0,1]$, the constants $C$ and $C_{\delta}$ depend on $\|g\|_{L_{b}^{2}}$ and $\|u\|_{\mathbb{W}_{b}}$, but are independent of $y, \delta>0$ is arbitrary and, in addition, the constant $C$ is independent of $\delta$.

We now recall that the integral $\int_{0}^{1}\|u(t)\|_{W_{\varphi, v}^{1,2}(\Omega)}^{2} d t \leq C\|u\|_{\mathbb{W}_{b}([0, T] \times \Omega)} \leq C_{1}$ uniformly with respect to $y \in \mathbb{R}$. Consequently, applying the Gronwall inequality (see Lemma 7.2 with $\left.Z(t):=t\left\|\nabla_{x}\left(\psi_{y} u(t)\right)\right\|_{L^{2}(\Omega)}^{2}\right)$ to (7.59), we deduce that

$T\left\|\nabla_{x}\left(\psi_{y} u(T)\right)\right\|_{L^{2}(\Omega)}^{2}+\alpha \int_{0}^{T} t\left\|\psi_{y} u(t)\right\|_{W^{2,2}(\Omega)}^{2} d t \leq C_{\delta}+\delta \int_{0}^{T} t\|u(t)\|_{W_{\varphi_{s, y}, 2,}^{2}(\Omega)}^{2} d t$

where $T \in[0,1]$, the constant $\delta>0$ is arbitrary, $\alpha>0$ and the constant $C_{\delta}$ depends on $\|u\|_{\mathbb{W}_{b}}$ and $\|g\|_{L_{b}^{2}}$, but is independent of $y \in \mathbb{R}$. 
Since the cut-off functions $\psi_{y}$ satisfy assumptions (7.2), then (7.60) implies the following estimate:

$$
T\left\|\nabla_{x} u(T)\right\|_{L^{2}\left(\Omega_{y}\right)}^{2}+\alpha \int_{0}^{T} t\|u(t)\|_{W^{2,2}\left(\Omega_{y}\right)}^{2} d t \leq C_{\delta}^{\prime}+\delta \int_{0}^{T} t\|u(t)\|_{W_{\varphi_{,}, y}^{2,2}(\Omega)}^{2} d t
$$

with some new constant $C_{\delta}^{\prime}$ which is also independent of $y \in \mathbb{R}$.

Multiplying inequality (7.61) by $\varphi_{\gamma, z}(y)$ with $\gamma<\varepsilon$, integrating over $y \in \mathbb{R}$ and using (2.12) with $q=1$, we obtain

$$
T\left\|\nabla_{x} u(T)\right\|_{L_{\varphi_{\gamma, z}}^{2}(\Omega)}^{2}+\alpha \int_{0}^{T} t\|u(t)\|_{W_{\varphi \gamma, z}^{2,2}(\Omega)}^{2} d t \leq C_{\delta}^{\prime \prime}+C \delta \int_{0}^{T} t\|u(t)\|_{W_{\varphi, z}^{2,2}(\Omega)}^{2} d t
$$

where all of the constants are independent of $z \in \mathbb{R}, \delta>0$ is still arbitrary and $\alpha$ is independent of $\delta$. Fixing finally $\delta>0$ to be small enough, we arrive at

$$
T\left\|\nabla_{x} u(T)\right\|_{L_{\varphi_{\gamma, z}}^{2}(\Omega)}^{2}+\alpha \int_{0}^{T} t\|u(t)\|_{W_{\varphi, z}^{2,2}(\Omega)}^{2} d t \leq C
$$

which implies (7.29) and Theorem 7.4 is proved.

The next corollary gives the analogue of Theorem 7.4 for more smooth initial data.

COROLlary 7.6. Let the assumptions of Theorem 6.6 be satisfied and let $u \in v_{c}+$ $\mathbb{W}_{b}([0, T] \times \Omega)$ be a weak solution of $(6.1)$ constructed in that theorem. Assume also that the initial data $u(0):=u_{0}$ belongs to the space $v_{c}+\mathcal{V}_{b}^{2}(\Omega)$. Then the solution $u$ is, in fact, more regular:

$$
u \in L^{\infty}\left([0, T], W_{b}^{1,2}(\Omega)\right) \cap L_{b}^{2}\left([0, T], W_{b}^{2,2}(\Omega)\right)
$$

and the following estimate holds:

$$
\|u(T)\|_{W^{1,2}\left(\Omega_{y}\right)}^{2}+\int_{T}^{T+1}\|u(\tau)\|_{W^{2,2}\left(\Omega_{y}\right)}^{2} d t \leq Q\left(\left\|u_{0}\right\|_{W_{b}^{1,2}(\Omega)}+\|g\|_{L_{b}^{2}([0, T] \times \Omega)}\right),
$$

where the monotonic function $Q$ is independent of $y \in \mathbb{R}$ and $T \in \mathbb{R}_{+}$.

Indeed, for $T \geq 1$ estimate (7.6) follows from Theorems 7.4 and 6.6 and for small $T$ it can be proved exactly as (7.29) (and even slightly simpler since we need not to use the multiplier $t$ in (7.30).

We conclude this section by establishing some useful regularity results for the pressure.

COROLlary 7.7. Under the assumptions of Corollary 7.6, the pressure $p(t, x)$ associated with the solution of the NS equations (6.1) is uniquely defined up to a constant depending on t and satisfies:

$$
\begin{aligned}
& \nabla_{x} p \in L_{b}^{2}([0, T] \times \Omega), \quad p-\mathbb{S} p \in L_{b}^{2}\left([0, T], W_{b}^{1,2}(\Omega)\right) \\
& \quad \times(\mathbb{S} p)\left(t, x_{1}\right)-(\mathbb{S} p)(t, 0) \in L_{b}^{2}\left([0, T], W_{b,\left(1+\left|x_{1}\right|\right)^{-1}}^{1,2}(\mathbb{R})\right)
\end{aligned}
$$


Moreover, the following estimate holds:

$$
\int_{t}^{t+1}\left(\left\|\nabla_{x} p(\tau)\right\|_{L^{2}\left(\Omega_{y}\right)}^{2}+\|p(\tau)-\operatorname{Sp} p(\tau)\|_{W^{1,2}\left(\Omega_{y}\right)}^{2}\right) d \tau \leq Q\left(\left\|u_{0}\right\|_{\mathcal{V}_{b}^{2}(\Omega)}+\|g\|_{L_{b}^{2}([0, T] \times \Omega)}\right)
$$

where the monotonic function $Q$ is independent of $y \in \mathbb{R}$ and $t \in[0, T-1]$.

Proof. We first note that, without loss of generality, one can assume that the external forces $g(t)=\Pi g(t)$ are divergent free. Indeed, otherwise, due to Theorem 4.4, we can write

$$
g(t)=\Pi g(t)+\nabla_{x} q(t)
$$

where, due to Theorem 4.4 and Remark 4.5, the function $q$ satisfies (7.65) and (7.66) and, consequently, can be included in the pressure $p$. For simplicity, we also assume that $S u_{1} \equiv 0$. The case of general nonzero flux can be considered analogously by the transformation of the dependent variable $v(t)=u(t)-v_{c}$.

Taking now the divergence from both sides of equation (6.1), we arrive at the following elliptic problem for $p$ :

$$
\left.\Delta_{x} p(t)=\operatorname{div}\left[\left(u(t), \nabla_{x}\right) u(t)\right]=\left(\partial_{x_{1}} u(t), \nabla_{x}\right) u_{1}(t)+\left(\partial_{x_{2}} u(t), \nabla_{x}\right) u_{2}(t)\right):=H_{u}(t)
$$

which should be endowed with the Newmann boundary condition:

$$
\left.\partial_{n} p\right|_{\partial \Omega}=-l_{n} \Delta_{x} u
$$

Moreover, averaging the first equation of (6.1) and taking into account that $\operatorname{div} u=0$ and $\mathbb{S} u_{1}=0$, we obtain the following version of the Bernoulli law:

$$
\partial_{x_{1}} \mathbb{S} p=-\mathbb{S}\left[\Delta_{x} u_{1}(t)\right]+\partial_{x_{1}} \mathbb{S}\left[u_{1}^{2}(t)\right]
$$

It is also not difficult to show that $\mathbb{S}\left[H_{u}(t)\right]=\partial_{x_{1}}^{2} \mathbb{S}\left[u_{1}^{2}(t)\right]$.

Furthermore, due to estimate (6.64) and interpolation inequality (2.39), we have

$$
\begin{aligned}
& \int_{t}^{t+1}\left\|H_{u}(\tau)\right\|_{L_{\phi_{\varepsilon, y}}^{2}(\Omega)}^{2} d \tau \leq C \int_{t}^{t+1}\left\|\nabla_{x} u(\tau)\right\|_{L_{\varphi_{\varepsilon} / 2, y}^{4}(\Omega)}^{4} d \tau \\
& \quad \leq C_{1}\left\|\nabla_{x} u\right\|_{L^{\infty}\left([t, t+1], L_{b}^{2}(\Omega)\right)}^{2} \int_{t}^{t+1}\|u(\tau)\|_{W_{\varphi_{\varepsilon}, 2, y}^{2,2}(\Omega)}^{2} d \tau \leq C_{2}
\end{aligned}
$$

where the constant $C_{2}$ depends on $u$ and $\varepsilon>0$, but is independent of $y \in \mathbb{R}$ and $t$.

Let us now define a function $p_{0}(t, x)$ as a solution of the following auxiliary problem:

$$
\Delta_{x} p_{0}=0,\left.\quad \partial_{n} p_{0}\right|_{\partial \Omega}=-\left.l_{n} \Delta_{x} u\right|_{\partial_{n} \Omega}, \quad \partial_{x_{1}} \mathbb{S} p_{0}=-\mathbb{S}\left[\Delta_{x} u\right]
$$

Since $\operatorname{div} \Delta_{x} u=0$, the solution of that equation exists (due to Proposition 3.7), defined up to a constant and satisfies

$$
\left\|\nabla_{x} p_{0}(t)\right\|_{L_{\varphi, y}^{2}(\Omega)}+\left\|p_{0}-S p_{0}\right\|_{W_{\varphi, y}^{1,2}(\Omega)} \leq C\left\|\Delta_{x} u(t)\right\|_{L_{\varphi_{\varepsilon, y}}^{2}(\Omega)}
$$


and its $x_{2}$-average obviously satisfies

$$
\left(S p_{0}\right)\left(t, x_{1}\right)-\left(\mathbb{S} p_{0}\right)(t, 0)=-\int_{0}^{x_{1}} \mathbb{S}\left[\Delta_{x} u_{1}(t, s, \cdot)\right] d s
$$

where $C(t):=\left(\mathbb{S} p_{0}\right)(t, 0)$ can be chosen arbitrarily. Formulae (7.73) and (7.74) together with estimate (7.64) show that the function $p_{0}$ satisfies (7.65) and (7.66).

So it only remains to consider the remainder $p_{1}:=p-p_{0}$ which should satisfy

$$
\Delta_{x} p_{1}(t)=H_{u}(t),\left.\quad \partial_{n} p_{1}\right|_{\partial \Omega}=0, \quad \partial_{x_{1}} \mathbb{S} p_{1}=\partial_{x_{1}} \mathbb{S}\left[u_{1}^{2}(t)\right] .
$$

Due to Proposition 3.5, the function $p_{1}-\mathfrak{S} p_{1}$ is uniquely defined from (7.75) and satisfies

$$
\left\|p_{1}(t)-S p_{1}(t)\right\|_{W_{\varphi_{\varepsilon, y}}^{2,2}(\Omega)} \leq C\left\|H_{u}(t)\right\|_{L_{\varphi_{\varepsilon, y}}^{2}(\Omega)}
$$

and the average $S p_{1}$ is again defined up to the $t$-dependent constant $C(t)$ and satisfies

$$
\left(\mathbb{S} p_{1}\right)\left(t, x_{1}\right)-\left(\mathbb{S} p_{1}\right)(t, 0)=\mathbb{S}\left[u_{1}^{2}\left(t, x_{1}, \cdot\right)\right] .
$$

Thus, due to (7.71), the component $p_{1}$ also satisfies (7.65) and (7.66) and Corollary 7.7 is proved.

REMARK 7.8. Corollary 7.7 shows that, in the regular case $u_{0} \in v_{c}+\mathcal{V}_{b}^{2}(\Omega)$, the Navier-Stokes equation (6.1) can be understood as equality in the space $L_{b}^{2}([0, T] \times \Omega)$. Moreover, we see from the last inclusion of (7.65) that the pressure $p(t, x)$ can grow at most linearly with respect to $x_{1} \rightarrow \infty$ in such a way that $\nabla_{x} p$ remains bounded. It is worth emphasizing that this result is sharp, since for the case of the classical Poiseuille flow the pressure grows indeed linearly with respect to $x_{1}$.

8. Dissipativity and attractors. In this concluding section, we verify that the Navier-Stokes problem (6.1) generates a dissipative dynamical system in the corresponding phase space and prove the existence of the associated global attractor. For simplicity, we restrict ourselves to consider the autonomous case only:

$$
g(t) \equiv g \in\left[L_{b}^{2}(\Omega)\right]^{2} .
$$

Then, due to Theorems 6.6 and 7.1, the Navier-Stokes problem (6.1) generates semigroups $S(t)=S_{c}(t)$ in the phase spaces

$$
\Phi:=\Phi_{c}=v_{c}+\mathcal{H}_{b}^{2}(\Omega)
$$

via the standard expression

$$
S(t) u_{0}:=u(t), \quad S\left(t_{1}+t_{2}\right)=S\left(t_{1}\right) \circ S\left(t_{2}\right), \quad t_{1}, t_{2} \geq 0 .
$$

The following theorem, which gives a dissipative estimate for the solutions of the Navier-Stokes problem, can be considered as the main result of the section.

THEOREM 8.1. Let the assumptions of Theorem 6.6 holds and, in addition, (8.1) be satisfied. Then, there exist positive constants $\alpha$ and $K$ and a monotonic function $Q$ such 
that, for every weak energy solution $u(t)$ of the Navier-Stokes problem (6.1)-(6.2), the following estimate holds:

$$
\|u(t)\|_{L_{b}^{2}(\Omega)} \leq Q\left(\|u(0)\|_{L_{b}^{2}(\Omega)}+\|g\|_{L_{b}^{2}(\Omega)}\right) e^{-\alpha t}+K\left(1+c^{3}+\|g\|_{L_{b}^{2}(\Omega)}^{2}\right)
$$

(we emphasize that the constant $K$ in (8.2) is independent of $t,\|u(0)\|_{L_{b}^{2}(\Omega)}$ and the flux $\left.c=\mathbb{S} u_{1}(0)\right)$.

Proof. In order to verify (8.4), it is sufficient to prove that the ball

$$
\mathcal{B}:=\left\{u_{0} \in\left[L_{b}^{2}(\Omega)\right]^{2}, \quad\left\|u_{0}\right\|_{L_{b}^{2}(\Omega)} \leq K\left(1+c^{3}+\|g\|_{L_{b}^{2}(\Omega)}^{2}\right)\right\}
$$

is an absorbing set for Navier-Stokes problem (6.1), i.e., that, for every bounded subset $B \subset \Phi$ there exists time $T=T\left(\|B\|_{\Phi},\|g\|_{L_{b}^{2}(\Omega)}\right)$ such that

$$
S(t) B \subset \mathcal{B}, \quad \forall t \geq T .
$$

Moreover, for simplicity, we restrict ourselves to consider only the case of zero flux $c=0$. The general case can be reduced to this particular one exactly as in Theorem 6.6.

The proof of embedding (8.6) requires a little more detailed analysis of basic a priori estimate (6.13) which we rewrite in the following more convenient way:

$$
\begin{aligned}
& \|u(t)\|_{L_{\theta_{\varepsilon}}^{2}(\Omega)}^{2}+\left(C_{1}-C_{2} \varepsilon\|u\|_{L^{\infty}\left([0, T], L_{\theta_{\varepsilon}}^{2}(\Omega)\right)}\right) \int_{0}^{T} e^{-\alpha|t-s|}\|u(s)\|_{W_{\theta_{\varepsilon}}^{1,2}(\Omega)}^{2} d s \\
& \quad \leq C_{3}^{2}\left(e^{-\alpha t}\|u(0)\|_{L_{\theta_{\varepsilon}}^{2}(\Omega)}+\|g\|_{L_{\theta_{\varepsilon}}^{2}(\Omega)}\right)^{2}
\end{aligned}
$$

where the positive constants $\alpha$ and $C_{i}, i=1,2,3$ are independent of $u, u_{0}, g, \varepsilon \rightarrow 0, T$ and $x_{0}$ (in order to deduce (8.7) from (6.13), it is sufficient to take $s=t$ in the left-hand side of it).

LEMMA 8.2. Let the assumptions of Theorem 6.5 holds and let, in addition, the initial data $u(0)$ for problem (6.1) satisfy

$$
C_{1}-2 C_{2} C_{3} \varepsilon\left(\|u(0)\|_{L_{\theta_{\varepsilon, x_{0}}}^{2}(\Omega)}+\|g\|_{L_{\theta_{\varepsilon, x_{0}}}(\Omega)}\right) \geq 0
$$

where all of the constants are the same as in (8.7). Then the associated weak energy solutions $u(t)$ of the Navier-Stokes problem (with zero flux $c=0$ ) satisfies

$$
\|u(t)\|_{L_{\theta_{\varepsilon, x_{0}}}^{2}(\Omega)} \leq C_{3}\left(\|u(0)\|_{L_{\theta_{\varepsilon, x_{0}}}^{2}(\Omega)} e^{-\alpha t}+\|g\|_{L_{\theta_{\varepsilon, x_{0}}}^{2}(\Omega)}\right),
$$

for all $t \geq 0$.

Proof. Indeed, estimate (8.7) implies (8.9) under the additional assumption that

$$
C_{1}-C_{2} \varepsilon\|u\|_{L^{\infty}\left(\mathbb{R}_{+}, L_{\theta_{\varepsilon}, x_{0}}^{2}(\Omega)\right)} \geq 0 .
$$

On the other hand, (8.9) gives

$$
\|u\|_{L^{\infty}\left(\mathbb{R}_{+}, L_{\theta_{\varepsilon}, x_{0}}^{2}(\Omega)\right)} \leq C_{3}\left(\|u(0)\|_{L_{\theta_{\varepsilon}, x_{0}}^{2}(\Omega)}+\|g\|_{L_{\theta_{\varepsilon}, x_{0}}^{2}(\Omega)}\right)
$$


which formally implies (8.10). Thus, using the continuity arguments (analogously to the proof of Theorem 6.5 ), we can verify that (8.9) really holds if the initial data satisfies (8.8) and Lemma (8.2) is proved.

We now note that, although (8.9) looks like a dissipative estimate (in the phase space $L_{\theta_{\varepsilon, x_{0}}}^{2}(\Omega)$ ), it is not sufficient to immediately finish the proof of the theorem, since the exponent $\varepsilon>0$ in it depends on $\|u(0)\|_{L_{b}^{2}(\Omega)}$ (through assumption (8.8)), namely,

$$
\varepsilon \leq \varepsilon_{0}:=C\left(\|u(0)\|_{L_{\theta_{\varepsilon, x_{0}}}^{2}(\Omega)}+\|g\|_{L_{\theta_{\varepsilon}, x_{0}}^{2}(\Omega)}+1\right)^{-2}
$$

for some positive $C$, see the proof of Theorem 6.5 .

Thus, we need to be able to increase the exponent $\varepsilon$ as $t \rightarrow \infty$; this is guaranteed by the following lemma.

LEMMA 8.3. Let the above assumptions hold. Then, for every bounded subset $B \subset \Phi$, there exists time $T=T(\|B\|,\|g\|)$ such that, for every $x_{0} \in \mathbb{R}$, we have

$$
C_{1}-2 C_{2} C_{3} \varepsilon\left(\|u(T)\|_{L_{\theta_{\varepsilon, x_{0}}}^{2}(\Omega)}+\|g\|_{L_{\theta_{\varepsilon}, x_{0}}^{2}(\Omega)}\right) \geq 0
$$

with $\varepsilon \geq \bar{\varepsilon}:=L\left(1+\|g\|_{L_{b}^{2}(\Omega)}\right)^{-2}$ (where the constant $L$ is independent of $u_{0}$ and $g$ ), if the initial data $u(0) \in B$.

Proof. We will prove the lemma by the iteration procedure. Indeed, let $T_{0}=0$ and $\varepsilon=\varepsilon_{0}$ be given by (8.12). Then, estimate (8.13) is satisfied with $\varepsilon=\varepsilon_{0}$ and $T=T_{0}$. Let us assume that (8.13) is already proven for some $T_{k}>0$ and $\varepsilon_{k}:=2^{k} \varepsilon_{0}<\bar{\varepsilon}$. Then, we only need to prove that there exists $T_{k+1}>T_{k}$ such that (8.13) is satisfied with $\varepsilon=\varepsilon_{k+1}:=2 \varepsilon_{k}$ and $T=T_{k+1}$. To this end, we note that

$$
\theta_{2 \varepsilon, x_{0}}(x):=\left(1+4 \varepsilon^{2}\left|x-x_{0}\right|^{2}\right)^{-1 / 2} \leq 2\left(1+\varepsilon^{2}\left|x-x_{0}\right|^{2}\right)^{1 / 2}=2 \theta_{\varepsilon, x_{0}}(x)
$$

and, consequently,

$$
\|v\|_{L_{\theta_{2 \varepsilon, x_{0}}}^{2}(\Omega)} \leq 2\|v\|_{L_{\theta_{\varepsilon, x_{0}}}^{2}(\Omega)} .
$$

Let us now fix $T_{k+1}>T_{k}$ in such a way that

$$
\left\|u\left(T_{k+1}\right)\right\|_{L_{\theta_{\varepsilon_{k}}, x_{0}}^{2}(\Omega)} \leq 2 C_{3}\|g\|_{L_{\theta_{\varepsilon_{k}}, x_{0}}^{2}(\Omega)},
$$

for all $u(t)$ such that $u(0) \in B$ (it is possible to do this due to our assumptions on $\varepsilon_{k}$ and "dissipative" estimate (8.9)). Estimates (8.14) and (8.15) together with (6.25) give

$$
\begin{aligned}
& \varepsilon_{k+1}\left(\left\|u\left(T_{k+1}\right)\right\|_{L_{\theta_{\varepsilon_{k+1}, x_{0}}}^{2}(\Omega)}+\|g\|_{L_{\theta_{\varepsilon_{k+1}}, x_{0}}^{2}(\Omega)}\right) \leq 4 \varepsilon_{k}\left(\left\|u\left(T_{k+1}\right)\right\|_{L_{\theta_{\varepsilon_{k}}, x_{0}}^{2}(\Omega)}+\|g\|_{L_{\theta_{\varepsilon_{k}, x_{0}}}^{2}(\Omega)}\right) \\
& \quad \leq 4\left(2 C_{3}+1\right) \varepsilon_{k}\|g\|_{L_{\theta_{\varepsilon_{k}}, x_{0}}^{2}(\Omega)} \leq 4\left(2 C_{3}+1\right) C \varepsilon_{k}^{1 / 2}\|g\|_{L_{b}^{2}(\Omega)} \\
& \quad \leq 4\left(2 C_{3}+1\right) C L^{1 / 2}\|g\|_{L_{b}^{2}(\Omega)}\left(1+\|g\|_{L_{b}^{2}(\Omega)}\right)^{-1} \leq 4 C\left(2 C_{3}+1\right) L^{1 / 2} .
\end{aligned}
$$

Thus, if the constant $L$ is small enough to satisfy

$$
C_{1}-8 C_{2} C C_{3}\left(2 C_{3}+1\right) L^{1 / 2} \geq 0
$$

then estimate (8.13) is satisfied with $T=T_{k+1}$ and $\varepsilon=\varepsilon_{k+1}=2 \varepsilon_{k}$. Thus, the iteration finishes the proof of the lemma. 
It is not difficult now to finish the proof of the theorem. Indeed, due to Lemma 8.3 and estimate (8.9), there exists $T=T(\|B\|,\|g\|)$ such that

$$
\|u(t)\|_{L_{\theta_{\varepsilon, x_{0}}}^{2}(\Omega)} \leq 2 C_{3}\|g\|_{L_{\theta_{\varepsilon, x_{0}}}^{2}(\Omega)}, \quad t \geq T
$$

holds with $\varepsilon \geq \bar{\varepsilon}:=L\left(1+\|g\|_{L_{b}^{2}(\Omega)}\right)^{-2}$ and uniformly with respect to $x_{0} \in \mathbb{R}$. Taking now supremum over $x_{0} \in \mathbb{R}$ from the both sides of inequality (8.16) and using again (6.25), we arrive at

$$
\|u(t)\|_{L_{b}^{2}(\Omega)} \leq 2 C_{3} C L^{-1 / 2}\|g\|_{L_{b}^{2}(\Omega)}\left(1+\|g\|_{L_{b}^{2}(\Omega)}\right), \quad t \geq T
$$

which shows that the ball (8.5) is really the absorbing set if $K \geq 2 C_{3} C L^{-1 / 2}$. Theorem 8.1 is proved.

REMARK 8.4. It is worth noting that the intermediate estimate (8.16) gives slightly more information on the solutions than the final one (8.17). Indeed, assume that $c=0$ and $g$ is square integrable $g \in\left[L^{2}(\Omega)\right]^{2}$. Then, instead of (6.25), we will have $\|g\|_{L_{\theta_{\varepsilon, x_{0}}}^{2}(\Omega)} \leq C\|g\|_{L^{2}(\Omega)}$ with the constant $C$ independent of $\varepsilon$. Thus, instead of (8.17), we will have the better estimate

$$
\|u(t)\|_{L_{b}^{2}(\Omega)} \leq 2 C_{3} C\|g\|_{L^{2}(\Omega)}, \quad t \geq T
$$

for the radius of the absorbing set (which grows now linearly with respect to $g$ in contrast to the quadratic growth rate in general case).

We are now in a position to prove the existence of a global attractor for semigroups (8.3) associated with the Navier-Stokes equation. We note however that, in contrast to the dissipative systems in bounded domains, in unbounded ones the global attractor is usually not compact in the initial phase space ( $\Phi$ in our case). That is the reason why we need to use the following weaker definition of a global attractor (following [6], [9], [18]).

Definition 8.5. A set $\mathcal{A} \subset \Phi$ is a locally compact (global) attractor for the semigroup $S(t): \Phi \rightarrow \Phi$ if the following assumptions are satisfied:

(1) the set $\mathcal{A}$ is bounded in $\Phi$ and compact in $\Phi_{l o c}:=v_{c}+\mathcal{H}_{l o c}^{2}(\bar{\Omega})$ (i.e., the restriction $\left.\mathcal{A}\right|_{\Omega_{1}}$ of the attractor $\mathcal{A}$ to any bounded subdomain $\Omega_{1}$ of $\Omega$ is compact in $L^{2}\left(\Omega_{1}\right)$;

(2) the set $\mathcal{A}$ is strictly invariant: $S(t) \mathcal{A}=\mathcal{A}$;

(3) the set $\mathcal{A}$ is an attracting set for the semigroup $S(t)$, i.e., for every neighborhood $\mathcal{O}(\mathcal{A})$ (in the local topology of the space $\Phi_{l o c}$ ) and every bounded (in $\Phi$ ) subset $B$, there exists time $T=T(\mathcal{O}, B)$ such that

$$
S(t) B \subset \mathcal{O}(\mathcal{A})
$$

for all $t \geq T$.

COROLLARY 8.6. Under the assumptions of Theorem 8.1, semigroup (8.3) associated with the Navier-Stokes problem (6.1)-(6.2) possesses locally compact attractor $\mathcal{A}=\mathcal{A}^{c}$ which is bounded in $v_{c}+\mathcal{V}_{b}^{2}(\Omega)$. Moreover, the following estimate holds:

$$
\|\mathcal{A}\|_{L_{b}^{2}(\Omega)} \leq K\left(1+c^{3}+\|g\|_{L_{b}^{2}(\Omega)}^{2}\right)
$$

where the constant $K$ is independent of $c$ and $g$. 
Proof. As usual, in order to verify the attractor's existence, we need to check the standard conditions, namely, the existence of a compact absorbing set and the continuity, see e.g. [6].

Indeed, due to Theorem 8.1, semigroup (8.2) possesses an absorbing set $\mathcal{B} \subset \Phi$ which is, however, not compact in the space $\Phi_{l o c}$. But, by Theorem 7.4, the set $S(1) \mathcal{B}$ is bounded in $v_{c}+\mathcal{V}_{b}^{2}(\Omega)$ and, consequently, is compact in $\Phi_{l o c}$. Thus, a compact absorbing set $\mathcal{B}_{1}:=S(1) \mathcal{B}$ for semigroup (8.2) is constructed. Moreover, due to Theorem 7.1, the operators $S(t): \mathcal{B}_{1} \rightarrow \Phi$ are continuous (in the topology of $\Phi_{\text {loc }}$ ) for every fixed $t>0$. Thus, due to the standard attractor existence theorem, semigroup $S(t)$ possesses a global attractor $\mathcal{A} \subset \mathcal{B}_{1} \cap \mathcal{B}$. Estimate (8.19) is now an immediate corollary of Theorem 8.1. Corollary 8.6 is proved.

To conclude the paper, we restore the physical parameters in the Navier-Stokes system (6.1), i.e. consider the problem

$$
\partial_{t} u+\left(u, \nabla_{x}\right) u=v \Delta_{x} u-\nabla_{x} p+g, \quad \operatorname{div} u=0
$$

in a strip $\Omega$ and study the dependence of the size of attractor on $v$.

COROLlary 8.7. The global attractor $\mathcal{A}=\mathcal{A}(c, g, v)$ of problem $(8.20)$ possesses the following estimate:

$$
\|\mathcal{A}\|_{L_{b}^{2}(\Omega)} \leq C v^{-3}\left(c^{3} v+\|g\|_{L_{b}^{2}(\Omega)}^{2}+v^{4}\right)
$$

where the constant $C$ is independent of $c, g$ and $v$.

Indeed, the scaling $t^{\prime}=v t, u^{\prime}=v^{-1} u$ reduces equation (8.21) to equation (6.1)(6.2) with $c^{\prime}=v^{-1} c$ and $g^{\prime}=v^{-2} g$. Since $\mathcal{A}^{\prime}=v^{-1} \mathcal{A}$, then (8.19) implies (8.21).

\section{REFERENCES}

1. L. Afendikov and A. Mielke, Multi-pulse solutions to Navier-Stokes problem between parallel planes, Z. Angew. Math. Phys. 52 (2001), 79-100.

2. L. Afendikov and A. Mielke, Dynamical properties of spatially non-decaying $2 \mathrm{D}$ NavierStokes flows with Kolmogorov forcing in an infinite strip, J. Math. Fluid Mech 7, suppl. 1 (2005), 51-67.

3. H. Amann, On the strong solvability of the Navier-Stokes equations, J. Math. Fluid. Mech. 2 (2000), 16-98.

4. A. Babin, Asymptotic expansions at infinity of a strongly perturbed Poiseuille flow, $A d v$. Soviet Math. 10 (1992), 1-83.

5. A. Babin, The attractor of a Navier-Stokes system in an unbounded channel-like domain, J. Dynam. Differential Equations 4 (1992), 555-584.

6. A. Babin and M. Vishik, Attractors of evolutionary equations (North Holland, Amsterdam, 1992).

7. A. Babin and M. Vishik, Attractors of partial differential evolution equations in an unbounded domain, Proc. Roy. Soc. Edinburgh Sect. A, 116 (1990), 221-243.

8. M. Efendiev, A. Miranville and S. Zelik, Infinite-dimensional exponential attractors for nonlinear reaction-diffusion systems in unbounded domains and their approximation, Proc. $R$. Soc. London A. 460 (2004), 1107-1129.

9. M. Efendiev and S. Zelik, Upper and lower bounds for the Kolmogorov entropy of the attractor for an RDE in an unbounded domain, J. Dyn. Dif. Eqns, 14 (2002) 369-403.

10. M. Efendiev M. and S. Zelik, The attractor for a nonlinear reaction-diffusion system in an unbounded domain, Comm. Pure Appl. Math. 54 (2001), 625-688. 
11. T. Gallay and C. Wayne, Invariant manifolds and the long-time asymptotics of the Navier-Stokes and vorticity equations in $\mathbb{R}^{2}$, Arch. Rat. Mech. Anal. 163 (2002), 209-258

12. Y. Giga, S. Matsui and O. Sawada, Global existence of two-dimensional Navier-Stokes flow with nondecaying initial velocity, J. Math. Fluid Mech. 3 (2001), 302-315.

13. G. Iooss and K. Kirchgässner, Water waves for small surface tension: an approach via normal form, Proc. Roy. Soc. Edinburgh Sect. A 122 (1992), 267-299.

14. G. Iooss and A. Mielke, Bifurcating time-periodic solutions of Navier-Stokes equations in infinite cylinders, J. Nonlinear Sci. 1 (1991), 107-146.

15. K. Kirchgässner, Wave solutions of reversible systems and applications, J. Diff. Eqns. 45 (1982), 113-127.

16. O. Ladyzhanskaya, V. Solonnikov and N. Uraltseva, Linear and quasilinear equations of parabolic type (Moscow: Izdat "Nanka", 1968).

17. A. Mielke, The Ginzburg-Landau equation in its role as a modulation equation, Handbook of dynamical systems (ed. by B. Fiedler), Elsevier (2002), 759-834.

18. A. Mielke and G. Schneider, Attractors for modulation equations on unbounded domains - existence and comparison, Nonlinearity 8 (1995), 743-768.

19. A. Mielke and S. Zelik, Infinite-dimensional hyperbolic sets and spatio-temporal chaos in reaction-diffusion systems in $\mathbb{R}^{n}$, J. Dynam. Diff. Eqns. 19 (2007), 333-389.

20. $\mathrm{H}$. Schaefer and M. Wolff, Topological vector spaces. Second edition. Graduate Texts in Mathematics, No. 3 (Springer-Verlag 1999).

21. G. Schneider, Global existence results for pattern formation processes in infinite cylindrical domains. Applications to 3D Navier-Stokes problem, J. Math. Pures. Appl. 78 (1999), $265-312$

22. G. Schneider and H. Uecker Almost global existence and transient self-similar decay for Poiseuille flow at criticality for exponentially long times, Phys. D, 185 (2003) 209-226. 1984).

23. R. Temam, Navier-Stokes equations, theory and numerical analysis (North-Holland,

24. R. Temam, Infinite-dimensional dynamical systems in mechanics and physics (SpringerVerlag 1997). 1978).

25. H. Triebel, Interpolation theory, function space, differential operators (North-Holland,

26. M. Vishik, Hydrodynamics in Besov spaces, Arch. Rat. Mech. Anal. 145 (1998), 197-214.

27. S. Zelik, Multiparametrical semigroups and attractors of reaction-diffusion systems in $\mathbb{R}^{n}$, Proc. Moscow Math. Soc. 65 (2004), 69-130.

28. S. Zelik, Attractors of reaction-diffusion systems in unbounded domains and their spatial complexity, Comm. Pure Appl. Math. 56 (2003), 584-637.

29. S. Zelik, The attractor for a nonlinear reaction-diffusion system in an unbounded domain and Kolmogorov's epsilon-entropy, Math. Nachr. 232 (2001), 129-179.

30. S. Zelik, The attractor for a nonlinear hyperbolic equation in an unbounded domain, Discrete Contin. Dynam Systems 7 (2001), 593-641.

31. S. Zelik, Spatial and temporal chaos in 2D Navier-Stokes equations in a strip, in preparation.

32. S. Zelik Weak spatially non-decaying solutions of 3D Navier-Stokes equations in cylindrical domains, Instability in Models Connected with Fluids Flow, International Math. Series, to appear. 